Göttinger Zentrum

für Biodiversitätsforschung und Ökologie

- Göttingen Centre for Biodiversity ANd Ecology -

\title{
Spatiotemporal patterns of insect diversity and multitrophic interactions across a tree diversity gradient in a Central European deciduous forest
}

Dissertation zur Erlangung des Doktorgrades der Mathematisch-Naturwissenschaftlichen Fakultäten der Georg-August-Universität Göttingen

\author{
vorgelegt von \\ Diplom-Biologin \\ Stephanie Sobek \\ aus \\ Hamm (Westf.)
}

Göttingen, 25. Juli, 2008 
Referent: Prof. Dr. Teja Tscharntke

Korreferent: Prof. Dr. Ingolf Steffan-Dewenter

Tag der mündlichen Prüfung: 04. September 2008 
„When you have seen one ant, one bird, one tree, you have not seen them all."

Edward O. Wilson 


\section{Contents}

Chapter 1: Introduction 1

Biodiversity \& forest ecosystems in a changing world 2

Umbrella project 3

Study area $\quad 4$

Study system $\quad 6$

$\begin{array}{ll}\text { Major hypotheses and chapter outline } & 10\end{array}$

Declaration of the author's own contribution to manuscripts with multiple authors $\quad 11$

References

Chapter 2: Spatiotemporal changes of beetle communities across a tree diversity gradient

$\begin{array}{ll}\text { Abstract } & 20\end{array}$

$\begin{array}{ll}\text { Introduction } & 21\end{array}$

$\begin{array}{ll}\text { Materials and Methods } & 23\end{array}$

Results 26

Discussion $\quad 33$

Acknowledgements 36

$\begin{array}{ll}\text { References } & 37\end{array}$

$\begin{array}{ll}\text { Appendix } & 43\end{array}$

Chapter 3: Room for more is in the mix: tree diversity drives spatiotemporal

及-diversity of true bugs (Heteroptera) 56

$\begin{array}{ll}\text { Abstract } & 57\end{array}$

$\begin{array}{ll}\text { Introduction } & 58\end{array}$

$\begin{array}{ll}\text { Materials and Methods } & 60\end{array}$

$\begin{array}{ll}\text { Results } & 63\end{array}$

$\begin{array}{ll}\text { Discussion } & 72\end{array}$

$\begin{array}{ll}\text { Acknowledgements } & 75\end{array}$ 
Chapter 4: Canopy vs. understory: How does tree diversity affect cavity-nesting bee and wasp communities and their natural enemies across forest strata?

Abstract

Introduction

Materials and Methods

Results

Discussion

Acknowledgements

References

Appendix

Chapter 5: Sapling herbivory, herbivores and predators across a semi-natural tree diversity gradient in Germany's largest connected deciduous forest

Abstract

Introduction

Materials and Methods

Results

Discussion

Acknowledgements

References

Appendix

Chapter 6: Discussion and conclusions

Summary/Zusammenfassung 
CHAPTER

1

Introduction 


\section{Biodiversity $\&$ forest ecosystems in a changing world}

Biodiversity research has become a key subject in modern ecology. Throughout the last decade, a plethora of studies has been conducted to elucidate the status quo and distribution of global biodiversity (Gaston 2000), to determine major threats (Purvis \& Hector 2000), to link biodiversity with ecosystem functioning and services (Loreau et al. 2001, Hooper et al. 2005, Kremen 2005, Balvanera et al. 2006), and to stop biodiversity loss by applying appropriate conservation measures (Perfecto et al. 1997, Rundlöf et al. 2008). What is biodiversity, and why has it recently gained major attention of scientists around the globe?

The term "biodiversity" combines different perceptions of variety among organisms. According to the United Nations' "Convention on Biological Diversity" (1992), it includes ecological diversity (=niche diversity), organismic diversity, and genetic diversity among individuals (Noss \& Cooperrider 1994, Schellnhuber et al. 1999). The UN conference on the environment and development in Rio de Janeiro (1992) also acknowledged biodiversity as a natural resource, and determined its conservation as a keystone task for future sustainable development (Haila \& Kouki 1994).

Biodiversity offers goods and services that are impossible to be replaced by man alone. If society remains unable to establish sustainability and fails in stopping the current biodiversity decline, ecosystem services such as pollination, provision of clean air and soil, biocontrol of pest outbreaks, continuity of biogeochemical cycles etc. will be lost (Ehrlich \& Ehrlich 1992). Besides these apparent driving forces of the environment, biodiversity also has economic implications and serves as resource for food, industrial production (e.g. timber, medical goods), and recreation (Ehrlich \& Ehrlich 1992).

Forest ecosystems, in tropical as well as in temperate regions, are believed to house the major proportion of global biodiversity (Carnus et al. 2006). As a result of global change, extensive timber logging, and conversion of forest to arable land for biofuel production, pristine forest habitats decline rapidly. According to the 2005 report of the Food and Agriculture Organisation of the United Nations (FAO), approximately 7.3 million hectares of forest are lost each year. Worldwide, only $30 \%$ of surface area is still covered by natural or planted forest, and especially Central Europe exhibits only a minute fraction of large-scale forest habitats (FAO 2005). In Germany, although $30 \%$ of land surface is forested, the proportion of old-growth natural forest equals zero (FAO 2005).

With the majority of pristine forest habitats already vanished and with prospect to future losses, it is of immediate importance to identify the contribution of the remaining forest 
habitats to global diversity and ecosystem functioning. Since the late Holocene, European beech (Fagus sylvatica L.) and other deciduous trees should be naturally predominant in Central Europe (Tinner \& Lotter 2006). Instead, for many years conifers have primarily been reforested (Puumalainen et al. 2003), and only recently sustainable forestry tries to reestablish a greater abundance and variety of deciduous tree species. For Central Europe and Germany in particular, the scope should thus lie on the last semi-natural deciduous forest habitats, which are scarce and widely scattered across a landscape otherwise dominated by agricultural land use.

Research on animal and plant communities in forest habitats has a long tradition in ecology, but nevertheless, blank spots remain on the map of global forest diversity. Although numerous examinations in tropical rainforests have illustrated that especially insects in the forest canopy are prime contributors to global biodiversity (Erwin 1982, Erwin 1988, Stork 1988, Novotny \& Basset 2005, Dial et al. 2006), canopy research in temperate deciduous forests in Central Europe has long been neglected, and only a handful of published studies on a limited selection of arthropod taxa exists. (Kampichler \& Teschner 2002, Goßner \& Bräu 2004, Goßner \& Ammer 2006, Goßner et al. 2007, Müller \& Goßner 2007).

In the present study, we try to fill these blanks by relating diversity and function of canopy and understory insect communities to a semi-natural tree diversity gradient ranging from simple beech to mixed deciduous forest stands located in the Hainich National Park (Thurinigia, Germany).

\section{Umbrella project}

The research conducted for this thesis was placed within the framework of the DFG (German Research Foundation) Research Training Group ("Graduiertenkolleg") 1086, "The role of biodiversity for biogeochemical cycles and biotic interactions in temperate deciduous forests". The multidisciplinary programme was aimed to link the work of $14 \mathrm{PhD}$ students, who independently examined various topics of temperate forest biodiversity and ecosystem functioning. A complete list of all parameters investigated can be found in Leuschner et. al. (in press), subprojects included: 
Abiotic stand characteristics, biogeochemical cycles, and productivity

Stand structure and abiotic conditions are primarily set by the tree species present in the canopy layer. Analyses included soil moisture, soil carbon and nutrient content, local stand climate, and management history. Biogeochemical processes were examined and modelled from the soil up to the canopy, including rainfall precipitation and water cycling, nutrient fluxes, sap flow and canopy transpiration, litter decomposition, soil respiration, and primary plant production (stem increment, leaf and fine root production, etc.).

\section{Biotic stand characteristic and interactions}

Again, focus was laid on examining animal and plant communities from the soil up to the high canopy. Investigations ranged from analysing abundance and diversity of mycorrhizal fungi, herb layer plants, tree seedlings, and full-grown trees to soil mesofauna (oribatid mites), macrofauna (earthworms, ground beetles, isopods, spiders, snails), and canopy arthropods. Results for the latter including beetles, true bugs, bees and wasps are described in detail in chapter 2 to 4 of the present thesis. Biotic interactions like parasitsm, herbivory of tree seedlings, and the abundance of herbivores and predators, as well as their functional relationships, are presented in chapter 4 and 5 .

\section{Economic evaluation}

As mentioned earlier in this chapter, biodiversity offers services and goods of high economic impact and monetary value. Hence, to make the multidisciplinary survey complete, the willingness-to-pay for insurance values maintained by diverse forest stands (e.g. biocontrol of pest outbreaks) was investigated in a choice experiment and by random interviews.

\section{Study area}

All investigations were conducted in the the Hainich National Park. The park was established in 1997 and is located in the federal state Thuringia (Germany) in temperate Central Europe. It is described as Germany's largest connected semi-natural deciduous forest, and covers a total area of 16,000 ha (Nationalpark Hainich; http://www.nationalpark-hainich.de). In the past, the region belonged to the territory of the former German Democratic Republic, and has been used for many years as a military training site by Russian occupants. The latter ensured that most of the forest remained unmanaged for at least 40 years. In 1997, 7,600 ha have been put under permanent conservation and been declared National Park. In 2005, 12 permanent 
research plots were set-up in the north-eastern part of the conservation zone close to the village Weberstedt, establishing a semi-naturally grown tree diversity gradient ranging from simple beech to mixed forest stands with up to 9 broadleaved tree species (Fig. 1.1 \& Fig 1.2).

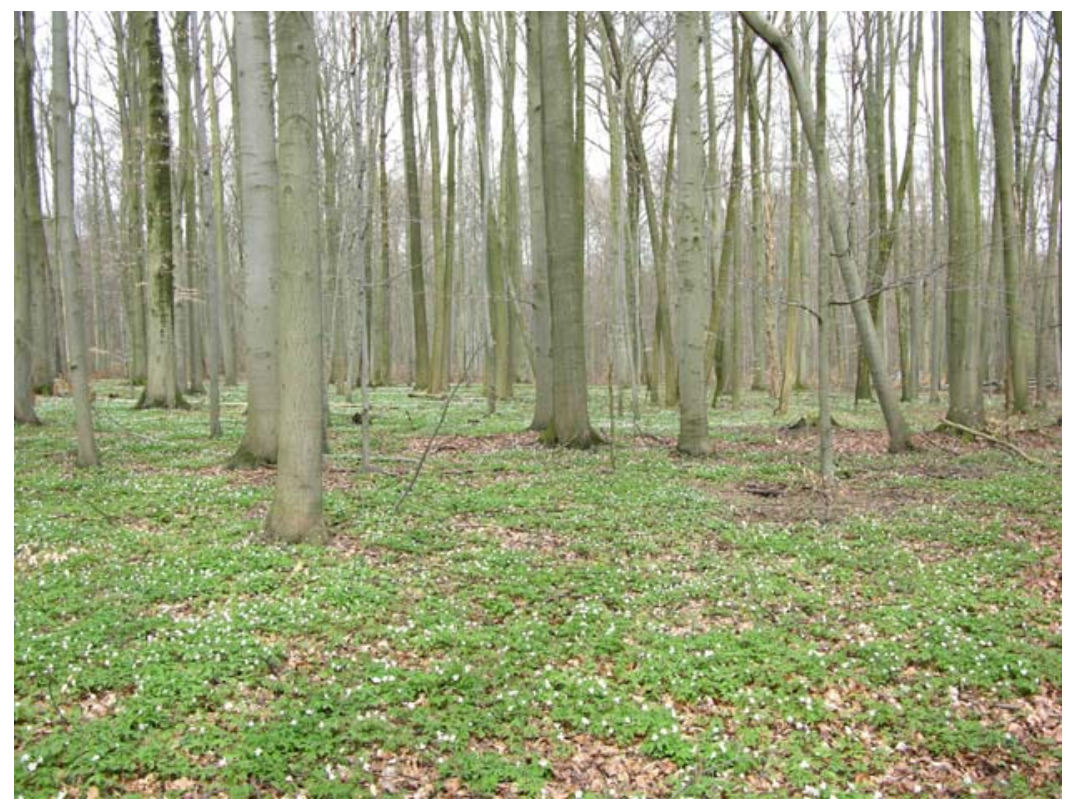

Fig. 1.1. Simple beech stand in the Hainich National Park in spring 2008 (photo: C. Scherber).

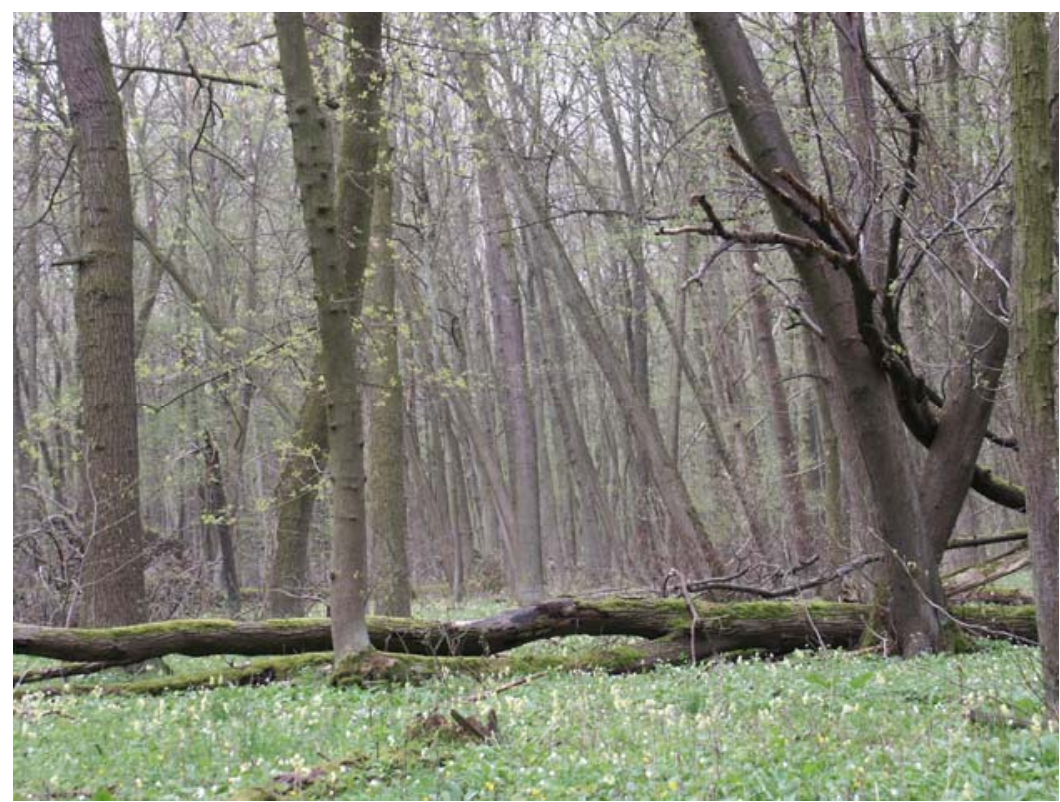

Fig. 1.2. Species-rich forest stand in the Hainich National Park in spring 2005 (photo: S. Schiele). 
The tree species composition of the Hainich National Park is highly exceptional, and unlike other forest habitats in Germany, conifers are a minority group and frequently logged to regain a late successional stage of semi-natural deciduous forest. One can find many stands dominated by European beech (Fagus sylvatica L.), other tree species like lime (Tilia sp.) and ash (Fraxinus excelsior L.) also appear frequently, and the mix is enriched with species like hornbeam (Carpinus betulus L.), maple (Acer campestre L., Acer platanoides L., Acer pseudoplatanus L.), cherry (Prunus avium L.), oak (Quercus robur L.) and elm (Ulmus glabra Huds.). Even the rare service tree (Sorbus torminalis L.) is present, and in total a maximum of 14 different tree species per hectare has been reported (Mölder et al. 2006).

\section{Study system}

\section{Insects in the forest canopy and understory}

Insects are the predominant taxon in terrestrial ecosystems by means of species richness, individual abundance, and biomass (Kremen 1993), and are of great importance for conservation management (Pearce \& Venier 2006, Underwood \& Fisher 2006). Estimations of global faunal diversity are closely interlinked with investigations on insect species richness, abundance, and host specifity (e. g. Erwin 1982, Erwin 1988, Erwin 1991, Longino 1994, Stork 1988), most of which have focused on forest canopy habitats in the tropics.

Species richness of arthropods has been reported to positively correlate with plant diversity (Gaston 1992, Siemann et al. 1998, Novotny et al. 2006). Habitat heterogeneity is usually enhanced in species-rich plant communities, resulting in a structurally more complex environment due to differences in plant architecture and increased availability of resources, offering a greater niche array and hence supporting a larger number of arthropod species (Humphrey et al. 1999, Hansen 2000, Hamer et al. 2003, Lassau \& Hochuli 2008). Although studies exist comparing the richness of arthropod communities of different tree species (Mawdsley \& Stork1997), far less is known about how communities change if different sets of tree species combinations are considered. Reason for this might be the lack of availability of semi-natural forest stands within a single study region that are comparable by means of climate, elevation, soil conditions, and management history. Recently conducted investigations used gradients, for example of management intensity, to estimate effects of anthropogenic disturbance and land use on forest insect communities (Müller et al. 2008), but information on effects of plant diversity gradients has so far only been gained from experiments (Vehviläinen et al. 2006, Vehviläinen et al. 2007). Making use of the semi- 
naturally established tree diversity gradient in the Hainich National Park, for the first time we examine species richness and distribution of three major taxa of canopy arthropods across a tree diversity gradient within a real-world forest ecosystem, and present data for beetles (Coleoptera), true bugs (Heteroptera), and cavity-nesting bees and wasps (Hymenoptera).

Beetles are believed to represent almost $40 \%$ of all arthropods worldwide (Grove \& Stork 2000). Up to now, 400,000 species have been described, 5,600 thereof in Central Europe (Honomichl 1998). Attributable to a long history of research on this order, most species are well described, and identification is eased by the availability of well-established keys. Beetles are economically and environmentally important as pest and predator species in agriculture and forestry, and have thus received major attention in biodiversity assessments of agricultural habitats, grassland habitats, tropical, boreal, and also temperate forests (e.g. Batary et al. 2007, Bos et al. 2007, Clough et al. 2007, Jacobs et al. 2007, McGeoch et al. 2007, Müller et al. 2008).

While the latter allows for a comparison of our data with existing material, information is vastly lacking for the heteropteran fauna in forest habitats. Although true bugs comprise sapsucking herbivores as well as predators, only very few studies on arboreal true bug communities exist (but see Goßner \& Bräu 2004, Goßner et al. 2006). Even for other ecosystems, data for true bugs are scarce, and especially data on temporal and spatial turnover of true bug diversity do not exist. Our data will provide new insights into the community structure of this formerly neglected group.

Beetles, as well as true bugs, were sampled with non-baited flight interception traps suspended in individual tree crowns (Fig. 1.3a, b), which is a common method for random sampling of flying insects (e.g. Simon \& Linsenmair 2001, Stork \& Grimbacher 2006, Grimbacher \& Catterall 2007), and allows for sufficient estimation of individual and species abundance within a certain area. 

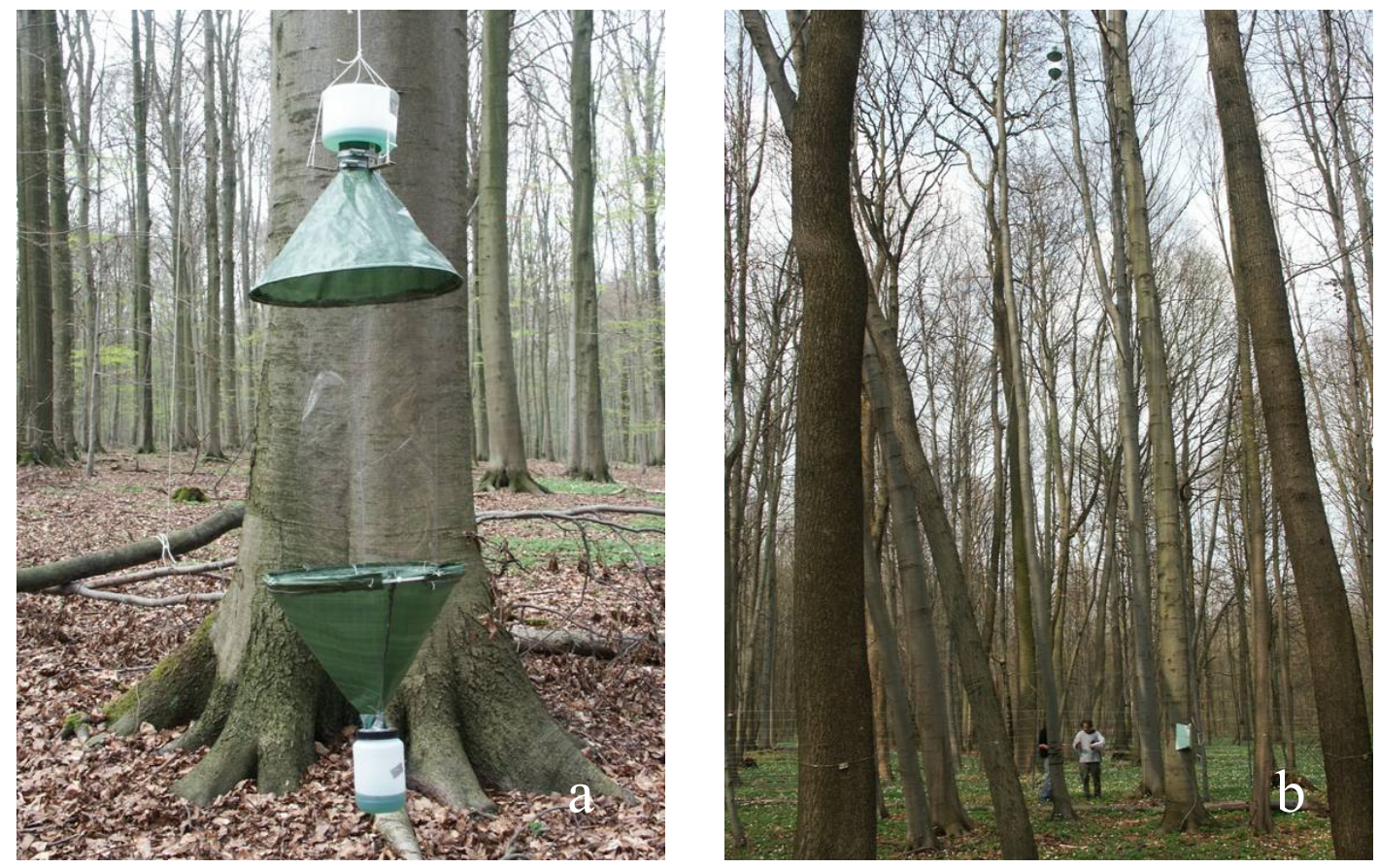

Fig. 1.3. a) Flight-interception trap, b) traps were mounted in the forest canopy (Photos: S. Schiele).

The inclusion of bees and wasps in our study resulted from a lack of data on how temperate forest habitats serve as a pool for hymenopteran diversity, including bees as pollinators of crop and wild plants, predatory wasps, and associated parasitoids. Due to anthropogenic habitat loss and fragmentation, pollinators like solitary bees decline rapidly, which has largescale consequences on plant survival (Biesmeijer et al. 2006). Various studies have dealt with pollinator abundance and species richness in agricultural habitats (e.g. Gathmann et al. 1994, Kruess \& Tscharntke 2002, Sheffield et al. 2008, Steffan-Dewenter \& Schiele 2008), and tropical agroforesty has also gained major attention recently (e.g. Klein et al. 2006, Tylianakis et al. 2006). Up to now, not much is known how bee pollinators and predatory wasps utilise forest habitats as source for food, shelter, and reproduction. Especially species-rich forests house a variety of flowering plants offering nectar and pollen resources (Mölder et al. 2006), which we expected to impact hymenopteran abundance and species richness, thus linking our observations to the provision of ecosystem services. Besides distribution of species richness and individual abundance, information on biotic interactions between parasitoids and hosts was included in the present study, which is of importance for the evaluation and understanding of diversity on an ecosystem scale (Montoya et al. 2003). The latter was accomplished by using trap nests instead of flight interception traps for sampling. Trap-nests provide a standardised method of sampling communities of cavity-nesting bees and wasps 
(Tscharntke et al. 1998), but up to now only two studies conducted in a temperate forest are known to the author (Taki et al. 2008b, Taki et al. 2008a), in which sampling was constricted to the understory. Here we give new insights in the spatial distribution of species by comparing vertical strata (canopy and understory habitats) across the tree diversity gradient (Fig. 1.4a,b).
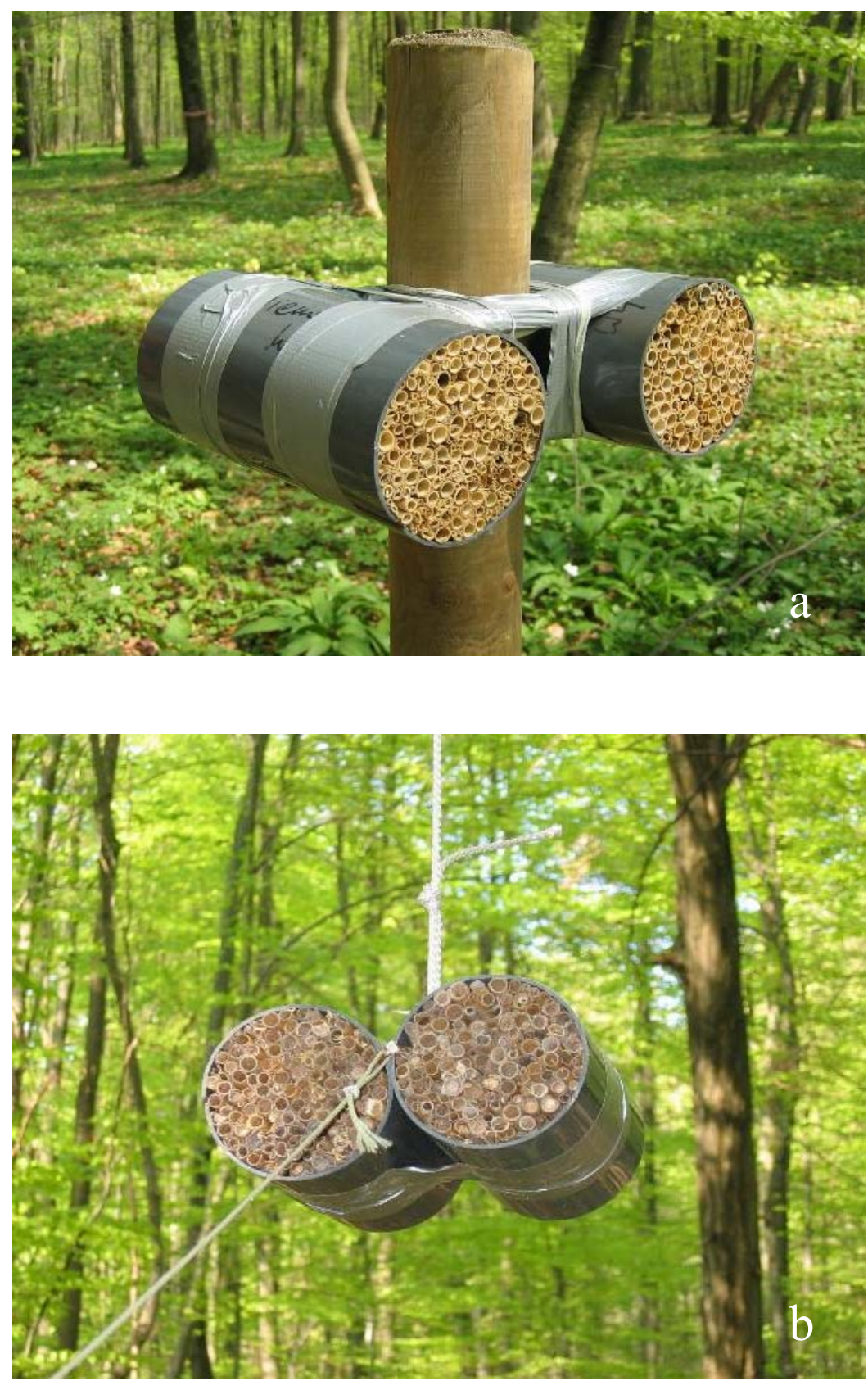

Fig. 1.4. Trap nests in the a) forest understory, b) forest canopy (photos: S. Schiele). 


\section{Herbivory of tree saplings}

While an assessment of arthropod diversity delivers valuable information for describing the fauna of a certain area, it is of special interest how arthropod communities interact with their environment, especially with regard to ecosystem functioning, e.g. resilience against pest outbreaks. Many recent biodiversity experiments have addressed this issue by investigating plant diversity-herbivory relationships. In various agricultural (Risch et al. 1983), grassland (Unsicker et al. 2006) and forest habitats (Jactel et al. 2005, Jactel et al. 2006, Jactel \& Brockerhoff 2007, Kaitaniemi et al. 2007), reduced herbivory was observed with increased plant diversity. However, some authors found no effect at all (Scherber et al. 2006) or even increased herbivory with increased plant diversity (Vehviläinen et al. 2006), and information on which patterns appear in natural forests is completely lacking. Utilising the tree diversity gradient, we examined insect herbivory on beech, Norway maple and sycamore maple saplings in the forest understory. For a most comprehensive survey of possible patterns and processes, we estimated damage caused by leaf-chewing, leaf-mining and gall-forming insects, and also observed the insect fauna (predators and herbivores) present on each sapling. Although previous studies dealing with insect herbivory have mostly included a survey of the associated herbivore fauna, the assessment of predator abundance and diversity has long been neglected, especially in forest habitats (but see Vehviläinen et al. 2008).

\section{Major hypotheses and chapter outline}

The general scope of this thesis was to analyse the effects of canopy tree diversity on insect diversity, community structure, and hebivory patterns across various spatiotemporal scales. In particular, we examined the following major hypotheses and questions:

Hypothesis 1: Insect species richness increases with increased canopy tree diversity.

- How does alpha, beta, and gamma diversity of insect communities change across spatiotemporal scales? (Chapter $2 \& 3$ )

- How is species richness in functional groups and feeding guilds distributed across the tree diversity gradient? (Chapter $2,3 \& 4$ )

- How are trophic interactions within insect communities mediated by tree diversity? (Chapter 4) 
Hypothesis 2: Herbivory decreases with increased canopy tree diversity.

- How does tree diversity impact insect herbivory on tree saplings in the forest understory (leaf area loss, galls, mines)? (Chapter 5)

- How is the abundance of predators and herbivores related to tree diversity and damage patterns? (Chapter 5)

\section{Declaration of the author's own contribution to manuscripts with multiple authors}

The chapters 2 to 5 are a series of manuscripts submitted to peer-reviewed journals. Stephanie Sobek is the overall author of all manuscripts presented in this thesis. She has personally developed the main ideas presented in the manuscripts, personally written all manuscripts, collected and analysed the data for all manuscripts, created tables, figures, and appendices, and contributed significantly to the sampling design. She is also responsible for correspondence with editors and reviewers.

\section{References}

Balvanera, P., Pfisterer, A.B., Buchmann, N., He, J.S., Nakashizuka, T., Raffaelli, D., \& Schmid, B. (2006) Quantifying the Evidence for Biodiversity Effects on Ecosystem Functioning and Services. Ecology Letters 9, 1146-1156.

Batary, P., Baldi, A., Szel, G., Podlussany, A., Rozner, I., \& Erdos, S. (2007) Responses of Grassland Specialist and Generalist Beetles to Management and Landscape Complexity. Diversity and Distributions 13, 196-202.

Biesmeijer, J.C., Roberts, S.P.M., Reemer, M., OhleMüller, R., Edwards, M., Peeters, T., Schaffers, A.P., Potts, S.G., Kleukers, R., Thomas, C.D., Settele, J., \& Kunin, W.E. (2006) Parallel Declines in Pollinators and Insect-Pollinated Plants in Britain and the Netherlands. Science 313, 351-354.

Bos, M.M., Steffan-Dewenter, I., \& Tscharntke, T. (2007) The Contribution of Cacao Agroforests to the Conservation of Lower Canopy Ant and Beetle Diversity in Indonesia. Biodiversity and Conservation 16, 2429-2444. 
Carnus, J.M., Parrotta, J., Brockerhoff, E., Arbez, M., Jactel, H., Kremer, A., Lamb, D., O'hara, K., \& Walters, B. (2006) Planted Forests and Biodiversity. Journal of Forestry 104, $65-77$.

Clough, Y., Kruess, A., \& Tscharntke, T. (2007) Organic Versus Conventional Arable Farming Systems: Functional Grouping Helps Understand Staphylinid Response. Agriculture Ecosystems \& Environment 118, 285-290.

Dial, R.J., Ellwood, M.D.F., Turner, E.C., \& Foster, W.A. (2006) Arthropod Abundance, Canopy Structure, and Microclimate in a Bornean Lowland Tropical Rain Forest. Biotropica 38, 643-652.

Ehrlich, P. R. \& Ehrlich, A. H. (1992) The Value of Biodiversity. Ambio 21, 219-22.

Erwin, T.L. (1982) Tropical Forests: Their Richness in Coleoptera and Other Arthropod Species. The Coleopterist's Bulletin 36, 74-75.

Erwin, T.L. (1988) The Tropical Forest Canopy- The Heart of Biotic Diversity. Biodiversity (ed. by E.O. Wilson), National Academy Press.

Erwin, T.L. (1991) How Many Species Are There - Revisited. Conservation Biology 5, 330333.

FAO, F.a.A.O.o.t.U.N. (2005) Global Forest Resources Assessment: Progress Towards a Sustainable Forest Management. Forest Paper 147 Rome, Italy.

Gaston, K.J. (1992) Regional Numbers of Insect and Plant Species. Functional Ecology 6, 243-247.

Gaston, K.J. (2000) Global Patterns in Biodiversity. Nature 405, 220-227.

Gathmann, A., Greiler, H.J., \& Tscharntke, T. (1994) Trap-Nesting Bees and Wasps Colonising Set-Aside Fields - Succession and Body-Size, Management by Cutting and Sowing. Oecologia 98, 8-14.

Goßner, M. \& Ammer, U. (2006) The Effects of Douglas-Fir on Tree-Specific Arthropod Communities in Mixed Species Stands With European Beech and Norway Spruce. European Journal of Forest Research 125, 221-235. 
Goßner, M. \& Bräu, M. (2004) Die Wanzen der Neophyten Douglasie (Pseudotsuga menziesii) und Amerikanischer Roteiche (Quercus rubra) im Vergleich zur Fichte und Tanne bzw. Stieleiche und Buche in südbayerischen Wäldern- Schwerpunkt arborikole Zönosen (Insecta: Heteroptera). Beiträge zur bayerischen Entomofaunistik 6, 217-235.

Goßner, M., Engel, K., \& Ammer, U. (2006) Effects of Selection Felling and Gap Felling on Forest Arthropod Communities: a Case Study in a Spruce-Beech Stand of Southern Bavaria. European Journal of Forest Research 125, 345-360.

Goßner, M., Liston, A., \& Spaeth, J. (2007) Sawflies in the Crowns of Native and Exotic Trees, Sampled With Flight-Interception Traps in Southern Germany (Hymenoptera : Symphyta). Entomologia Generalis 30, 273-282.

Grimbacher, P.S. \& Catterall, C.P. (2007) How Much Do Site Age, Habitat Structure and Spatial Isolation Influence the Restoration of Rainforest Beetle Species Assemblages? Biological Conservation 135, 107-118.

Grove, S.J. \& Stork, N.E. (2000) An Inordinate Fondness for Beetles. Invertebrate Taxonomy 14, 733-739.

Haila Y. \& Kouki J. (1994) The Phenomenon of Biodiversity in Conservation Biology. Annales Zoologici Fennici 31, 5-18.

Hamer, K.C., Hill, J.K., Benedick, S., Mustaffa, N., Sherratt, T.N., Maryati, M., \& Chey, V.K. (2003) Ecology of Butterflies in Natural and Selectively Logged Forests of Northern Borneo: the Importance of Habitat Heterogeneity. Journal of Applied Ecology 40, 150-162.

Hansen, R.A. (2000) Effects of Habitat Complexity and Composition on a Diverse Litter Microarthropod Assemblage. Ecology 81, 1120-1132.

Honomichl, K. (1998) Biologie und Ökologie der Insekten Gustav Fischer Verlag, Stuttgart.

Hooper, D.U., Chapin, F.S., Ewel, J.J., Hector, A., Inchausti, P., Lavorel, S., Lawton, J.H., Lodge, D.M., Loreau, M., Naeem, S., Schmid, B., Setala, H., Symstad, A.J., Vandermeer, J., \& Wardle, D.A. (2005) Effects of Biodiversity on Ecosystem Functioning: a Consensus of Current Knowledge. Ecological Monographs 75, 3-35.

Humphrey, J.W., Hawes, C., Peace, A.J., Ferris-Kaan, R., \& Jukes, M.R. (1999) 
Relationships Between Insect Diversity and Habitat Characteristics in Plantation Forests. Forest Ecology and Management 113, 11-21.

Jacobs, J.M., Spence, J.R., \& Langor, D.W. (2007) Influence of Boreal Forest Succession and Dead Wood Qualities on Saproxylic Beetles. Agricultural and Forest Entomology 9, 3-16.

Jactel, H., Brockerhoff, E., \& Duelli, P. (2005) A Test of the Biodiversity-Stability Theory: Meta-Analysis of Tree Species Diversity Effects on Insect Pest Infestations, and ReExamination of Responsible Factors. Forest Diversity and Function. Temperate and Boreal Systems. Ecological Studies 176 (ed. by M. Scherer-Lorenzen, Ch. Körner, and E.-D. Schulze), pp. 235-262. Springer-Verlag, Berlin.

Jactel, H. \& Brockerhoff, E.G. (2007) Tree Diversity Reduces Herbivory by Forest Insects. Ecology Letters 10, 835-848.

Jactel, H., Menassieu, P., Vetillard, F., Gaulier, A., Samalens, J.C., \& Brockerhoff, E.G. (2006) Tree Species Diversity Reduces the Invasibility of Maritime Pine Stands by the Bast Scale, Matsucoccus feytaudi (Homoptera : Margarodidae). Canadian Journal of Forest Research-Revue Canadienne De Recherche Forestiere 36, 314-323.

Kaitaniemi, P., Riihimaki, J., Koricheva, J., \& Vehvilainen, H. (2007) Experimental Evidence for Associational Resistance Against the European Pine Sawfly in Mixed Tree Stands. Silva Fennica 41, 259-268.

Kampichler, C. \& Teschner, M. (2002) The Spatial Distribution of Leaf Galls of Mikiola fagi (Diptera : Cecidomyiidae) and Neuroterus quercusbaccarum (Hymenoptera : Cynipidae) in the Canopy of a Central European Mixed Forest. European Journal of Entomology 99, 79-84.

Klein, A.M., Steffan-Dewenter, I., \& Tscharntke, T. (2006) Rain Forest Promotes Trophic Interactions and Diversity of Trap-Nesting Hymenoptera in Adjacent Agroforestry. Journal of Animal Ecology 75, 315-323.

Kremen, C. (1993) Terrestrial Arthropod Assemblages: Their Use in Conservation Planning. Conservation Biology 7, 796-808.

Kremen, C. (2005) Managing Ecosystem Services: What Do We Need to Know About Their Ecology? Ecology Letters 8, 468-479. 
Kruess, A. \& Tscharntke, T. (2002) Grazing Intensity and the Diversity of Grasshoppers, Butterflies, and Trap-Nesting Bees and Wasps. Conservation Biology 16, 1570-1580.

Lassau, S.A. \& Hochuli, D.F. (2008) Testing Predictions of Beetle Community Patterns Derived Empirically Using Remote Sensing. Diversity and Distributions 14, 138-147.

Leuschner, C., Jungkunst, H.F., \& Fleck, S. (in press) Studying the Functional Role of Tree Diversity in Forests: the Pros and Cons of Synthetic Stands and Across-Site Comparisons in Established Forests. Basic and Applied Ecology.

Longino, J.T. (1994) How to Measure Arthropod Diversity in a Tropical Rain Forest. Biology International 28, 3-13.

Loreau, M., Naeem, S., Inchausti, P., Bengtsson, J., Grime, J.P., Hector, A., Hooper, D.U., Huston, M.A., Raffaelli, D., Schmid, B., Tilman, D., \& Wardle, D.A. (2001) Ecology Biodiversity and Ecosystem Functioning: Current Knowledge and Future Challenges. Science 294, 804-808.

Mawdsley, N.A. \& Stork, N.E. (1997) Host-Specificity and the Effective Specialisation of Tropical Canopy Beetles. Canopy Arthropods (ed. by N.E. Stork, J. Adis, and R.K. Didham), pp. 104-129. Chapman \& Hall, London.

McGeoch, M.A., Schroeder, M., Ekbom, B., \& Larsson, S. (2007) Saproxylic Beetle Diversity in a Managed Boreal Forest: Importance of Stand Characteristics and Forestry Conservation Measures. Diversity and Distributions 13, 418-429.

Mölder, A., Bernhardt-Römermann, M., \& Schmidt, W. (2006) Forest Ecosystem Research in Hainich National Park (Thuringia): First Results on Flora and Vegetation in Stands with Contrasting Tree Species Diversity. Waldökologie online 3, 83-99.

Montoya, J.M., Rodríguez, M.A., \& Hawkins, B.A. (2003) Food Web Complexity and Higher-Level Ecosystem Services. Ecology Letters 6, 587-593.

Müller, J., Bussler, H., \& Kneib, T. (2008) Saproxylic Beetle Assemblages Related to Silvicultural Management Intensity and Stand Structures in a Beech Forest in Southern Germany. Journal of Insect Conservation 12, 107-124.

Müller, J. \& Goßner, M. (2007) Single Host Trees in a Closed Forest Canopy Matrix: a 
Highly Fragmented Landscape? Journal of Applied Entomology 131, 613-620.

Noss, R. F., Cooperrider A. Y. (1994) Saving nature's legacy: protecting and restoring biodiversity Island Press, Covelo, California.

Novotny, V. \& Basset, Y. (2005) Review - Host Specificity of Insect Herbivores in Tropical Forests. Proceedings of the Royal Society B-Biological Sciences 272, 1083-1090.

Novotny, V., Drozd, P., Miller, S.E., Kulfan, M., Janda, M., Basset, Y., \& Weiblen, G.D. (2006) Why Are There So Many Species of Herbivorous Insects in Tropical Rainforests? Science 313, 1115-1118.

Pearce, J.L. \& Venier, L.A. (2006) The Use of Ground Beetles (Coleoptera : Carabidae) and Spiders (Araneae) as Bioindicators of Sustainable Forest Management: a Review. Ecological Indicators 6, 780-793.

Perfecto, I., Vandermeer, J., Hanson, P., \& Cartín, V. (1997) Arthropod Biodiversity Loss and the Transformation of a Tropical Agro-Ecosystem. Biodiversity and Conservation 6, 935-945.

Purvis, A. \& Hector, A. (2000) Getting the Measure of Biodiversity. Nature 405, 212-219.

Puumalainen, J., Kennedy, P., \& Folving, S. (2003) Monitoring Forest Biodiversity: a European Perspective With Reference to Temperate and Boreal Forest Zone. Journal of Environmental Management 67, 5-14.

Risch, S.J., Andow, D., \& Altieri, M.A. (1983) Agroecosystem Diversity and Pest Control: Data, Tenntative Conclusions, and New Research Directions . Environmental Entomology 12, 625-629.

Rundlöf, M., Bengtsson, J., \& Smith, H.G. (2008) Local and Landscape Effects of Organic Farming on Butterfly Species Richness and Abundance. Journal of Applied Ecology 45, 813820.

Schellnhuber, H.-J., Kokott, J., Beese, F. O., Fraedrich, K., Klemmer, P., Kruse-Graumann, L., Neumann, C., Renn, O., Schulze, E-D., Tilzer, M., Velsinger, P., \& Zimmermann, H. (1999) WBGU (German Advisory Council on Global Change). World in transition: conservation and sustainable use of the biosphere Earthscan Publications Ltd. 
Scherber, C., Mwangi, P.N., Temperton, V.M., Roscher, C., Schumacher, J., Schmid, B., \& Weisser, W.W. (2006) Effects of Plant Diversity on Invertebrate Herbivory in Experimental Grassland. Oecologia 147, 489-500.

Sheffield, C.S., Kevan, P.G., Westby, S.M., \& Smith, R.F. (2008) Diversity of Cavity-Nesting Bees (Hymenoptera : Apoidea) Within Apple Orchards and Wild Habitats in the Annapolis Valley, Nova Scotia, Canada. Canadian Entomologist 140, 235-249.

Siemann, E., Tilman, D., Haarstad, J., \& Ritchie, M. (1998) Experimental Tests of the Dependence of Arthropod Diversity on Plant Ddiversity. The American Naturalist 152, 738750 .

Simon, U. \& Linsenmair, K.E. (2001) Arthropods in Tropical Oaks: Differences in Their Spatial Distributions Within Tree Crowns. Plant Ecology 153 , 179-191.

Steffan-Dewenter, I. \& Schiele, S. (2008) Do Resources or Natural Enemies Drive Bee Population Dynamics in Fragmented Habitats? Ecology 89, 1375-1387.

Stork, N.E. (1988) Insect Diversity: Facts, Fiction and Speculation. Biological Journal of the Linnean Society 35, 321-337.

Stork, N.E. \& Grimbacher, P.S. (2006) Beetle Assemblages From an Australian Tropical Rainforest Show That the Canopy and the Ground Strata Contribute Equally to Biodiversity. Proceedings of the Royal Society B-Biological Sciences 273, 1969-1975.

Taki, H., Kevan, P.G., Viana, B.F., Silva, F.O., \& Buck, M. (2008a) Artificial Covering on Trap Nests Improves the Colonisation of Trap-Nesting Wasps. Journal of Applied Entomology 132, 225-229.

Taki, H., Viana, B.F., Kevan, P.G., Silva, F.O., \& Buck, M. (2008b) Does Forest Loss Affect the Communities of Trap-Nesting Wasps (Hymenoptera : Aculeata) in Forests? Landscape Vs. Local Habitat Conditions. Journal of Insect Conservation 12, 15-21.

Tinner, W. \& Lotter, A.F. (2006) Holocene Expansions of Fagus silvatica and Abies alba in Central Europe: Where Are We After Eight Decades of Debate? Quaternary Science Reviews 25, 526-549. 
Tscharntke, T., Gathmann, A., \& Steffan-Dewenter, I. (1998) Bioindication Using TrapNesting Bees and Wasps and Their Natural Enemies: Community Structure and Interactions. Journal of Applied Ecology 35, 708-719.

Tylianakis, J.M., Klein, A.M., Lozada, T., \& Tscharntke, T. (2006) Spatial Scale of Observation Affects Alpha, Beta and Gamma Diversity of Cavity-Nesting Bees and Wasps Across a Tropical Land-Use Gradient. Journal of Biogeography 33, 1295-1304.

Underwood, E.C. \& Fisher, B.L. (2006) The Role of Ants in Conservation Monitoring: if, When, and How. Biological Conservation 132, 166-182.

Unsicker, S.B., Baer, N., Kahmen, A., Wagner, M., Buchmann, N., \& Weisser, W.W. (2006) Invertebrate Herbivory Along a Gradient of Plant Species Diversity in Extensively Managed Grasslands. Oecologia 150, 233-246.

Vehviläinen, H., Koricheva, J., Ruohomäki, K., Johansson, T., \& Valkonen, S. (2006) Effects of Tree Stand Species Composition on Insect Herbivory of Silver Birch in Boreal Forests. Basic and Applied Ecology 7, 1-11.

Vehviläinen, H., Koricheva, J., \& Ruohomäki, K. (2007) Tree Species Diversity Influences Herbivore Abundance and Damage: Meta-Analysis of Long-Term Forest Experiments. Oecologia 152, 287-298.

Vehviläinen, H., Koricheva, J., \& Ruohomäki, K. (2008) Effects of Stand Tree Species Composition and Diversity on Abundance of Predatory Arthropods. Oikos. Online Early. 


\section{CHAPTER}

2

\section{Spatiotemporal changes of beetle communities across a tree diversity gradient}

S. Sobek, I. Steffan-Dewenter, C. Scherber, T. Tscharntke (submitted) 


\begin{abstract}
Plant and arthropod diversity are often related, but data on the role of mature tree diversity on canopy insect communities are only fragmentary. Here we compare species richness of canopy beetles across a tree diversity gradient ranging from mono-dominant beech to mixed stands within a deciduous forest, and analyse community composition changes across space and time. We used flight interception traps to assess the beetle fauna of various tree species, and applied additive partitioning to examine spatiotemporal patterns of diversity. Species richness of beetle communities increased across the tree diversity gradient from 99 to 181 species per forest stand. Intra- and interspecific spatial turnover among trees contributed more than temporal turnover among months to the total $\gamma$-beetle diversity of the sampled stands. However, due to parallel increases in the number of habitat generalists and the number of species in each feeding guild (herbivores, predators, fungivores), no proportional changes in community composition could be observed. If only beech trees were analysed across the gradient, patterns were similar, but temporal (monthly) species turnover was higher compared to spatial turnover among trees, and not related to tree diversity. The changes in species richness and community composition across the gradient can be explained by habitat heterogeneity, which increased with the mix of tree species. We conclude that understanding temporal and spatial species turnover is the key to understanding biodiversity patterns. Monodominant beech stands are insufficient to fully conserve the regional species richness of the remaining semi-natural deciduous forest habitats in Central Europe, and analysing beech alone would have resulted in the misleading conclusion that temporal turnover contributes more to beetle diversity than spatial turnover among different tree species or individuals.
\end{abstract}

Key words: Beta diversity, biodiversity conservation, canopy arthropods, Fagus sylvatica L., functional groups, habitat heterogeneity 


\section{Introduction}

Being the most species-rich and prevalent insect taxon worldwide (Grove \& Stork 2000), beetles (Coleoptera) contribute greatly to biodiversity in forest habitats and play various roles in ecosystem dynamics and functioning (Erwin 1997, Lassau et al. 2005). Among them are numerous forest pests (e. g. Scolytidae, Curculionidae), as well as effective predators (e. g. Carabidae, Cleridae, Coccinellidae) capable of top-down biocontrol (Reeve 1997). Only few current studies exist that directly compare beetle communities of different tree species (i. e. Wagner 2000, Hulcr et al. 2007), and due to limited availability of comparable forest stands of different diversity within the same forest neighbourhood, so far these examinations mostly focused on local single-stand observations (but see Gering \& Christ 2000). Sometimes accessibility of habitats can be a problem as well, especially if sampling is not constrained to the understorey, but also includes the forest canopy.

The forest canopy is known as a major pool of global insect diversity (Erwin 1988, Stork 1988, Novotny \& Basset 2005), and especially in the tropics beetles are one of the beststudied taxonomic groups in this habitat. In contrast to the vast number of studies conducted in tropical forest canopies, canopy research in temperate deciduous forests is still fragmentary. Especially, turnover in space and time has largely been neglected (Ulyshen \& Hanula 2007), although it might be of importance for determining forest insect diversity (Hirao et al. 2007). For temperate deciduous forests, Gering \& Crist (2000) have demonstrated the importance of tree species, season, and spatial variability for structuring species richness and abundance patterns of beetles, and have also shown that particularly in late summer tree species identity plays a major role in determing beetle species richness. They also emphasised that with regard to spatial dependency of tree-dwelling arthropod communities, temperate forests remain vastly unexplored.

Due to host specificity (Erwin 1982, Stork 1988) and habitat preferences, species-rich forests can be expected to exhibit a greater diversity of beetle species, and in experimental forest habitats, tree diversity has been shown to positively affect arthropod species richness (Vehviläinen et al. 2008). As a measure of host speficifity in insects, May (1990) introduced the term "effective specialisation". Effective specialisation sensu May (1990) is defined as the weighted quantity of an insect assemblage specialised to a certain host tree species. However, effective specialisation of beetles has been demonstrated to strongly depend on the spatial scales considered (Gering et al. 2007). Moreover, community analysis across various spatiotemporal scales also increases the chance that ecologically relevant scales are included, 
which might otherwise be overlooked (Huston 1999). In particular for canopy beetles, community interaction takes place in individual tree crowns (Gering \& Crist 2002), and sample-based $\alpha$-diversity can be utilised as a snapshot of this community at a given time. Even trees in close proximity to each other $(<1 \mathrm{~km})$ might vary significantly in their species composition (Gering et al. 2003). Identifying community composition at various spatiotemporal scales can thus be of value for biodiversity conservation and forest management, by pinpointing species unique to certain scales and by preserving them accordingly (Summerville et al. 2003a). Hence, the interrelation of tree species, space and time needs to be disentangled, which can be accomplished by comparing beetle community patterns in highly diverse forest stands with patterns in species-poor stands within one and the same forest ecoregion.

Here we examine species richness and composition of canopy beetle communities across a tree diversity gradient ranging from mono-dominant beech to mixed stands in Germany's largest remaining deciduous forest habitat, the Hainich National Park. Because no studies up to date exist that focus on Fagus sylvatica in forest stands of different diversity, we compare beetle communities of various tree species with beetle communities of single beech trees across the gradient. Being the most important tree species in Central Europe (Gessler et al. 1998), it is of special interest to what extent beech contributes to hosting local and regional arthropod diversity. Due to more acidic soils, thicker leaf layer and often lesser light permeablility, beech dominated stands are usually characterised by low plant diversity (Ellenberg 1996, Mölder et al. 2008). They also appear generally homogenous (Kenderes et al. 2008) and less structurally complex compared to diverse forests.

Whereas up to now a plethora of studies conducted on forest beetle communities in Europe has focused on saproxylic species only (many of them reviewed in Grove (2002), Davies et al. (2008)), but to a lesser extent included other functional groups, we carry out a complete survey of all captured species and analyse community composition of the different stands. We use additive partitioning (Lande 1996, Veech et al. 2002, Crist et al. 2003) to not only account for overall species richness per forest stand, but to also include spatial and temporal differences in the observed patterns.

Specifically, we test the following hypotheses: (1) Due to increased habitat heterogeneity, species-rich forest stands house a greater number of beetle species ( $\gamma$-diversity) compared to mono-dominant beech stands. The presence of a wide variety of different tree species enhances habitat complexity and thus niche availability, which should support a greater array of beetle species. The same accounts for (2) species turnover ( $\beta$-diversity), which we expect 
to be higher in species-rich forest stands. The various tree species and other plants in diverse forests might attract a greater variety of feeding specialists, which in turn could increase predator diversity. Several beetle species show a high degree of host specialisation and are closely associated with certain tree species, for example oak (Müller \& Goßner 2007), with a decreased chance of encounter on non-host trees. Hence, we assume that in the most diverse forest stands (3) spatial turnover $\left(\beta_{\text {space}}\right)$ between trees contributes more to overall $\gamma$-diversity than temporal (monthly) turnover $\beta_{\text {time. }}$ (4) In contrast, spatiotemporal patterns of beetle diversity on beech alone should remain constant across the tree diversity gradient with a greater contribution of monthly turnover to the total diversity, unless beetle species richness on single beech trees in mixed forest stands increases due to spill-over effects. Due to differences in life-cycle and voltinism, beetles show a strong seasonality and several species are only actively dispersing as adults for a short period of time (Gaylord et al. 2006), thereby increasing temporal turnover. The latter is likely to be more prominent in mono-dominant beech forests, where spatial turnover between conspecific trees is expected to contribute less to overall diversity.

\section{Methods}

\section{Study area \& field sites}

Trees were sampled in Germany's largest coherent semi-natural broadleaved forest, the Hainich National Park, Thuringia. Established in 1997, 7,600 ha of forest (of a total area of 16,000 ha) are currently under protection (Nationalpark Hainich; http://www.nationalparkhainich.de), while the surrounding area is dominated by arable land. Sampled forest stands are located in the north-eastern part of the protected zone south of the village Weberstedt. The region has a temperate climate, with an average temperature of $7.5{ }^{\circ} \mathrm{C}$ and a mean precipitation of $590 \mathrm{~mm}$ (1973-2004, Deutscher Wetterdienst). The average annual temperature of the area in 2005 was $9{ }^{\circ} \mathrm{C}$, annual precipitation in 2005 was $601 \mathrm{~mm}$ (Meteomedia 2005). The predominant soil type is stagnic luvisol on loess-limestone as parent material.

Various deciduous tree species grow in the mild climate of the research area. In the examined forest stands, dominant tree species are beech (Fagus sylvatica L.), lime (Tilia platyphyllos Scop., Tilia cordata L.) and ash (Fraxinus excelsior L.). Conifers occur only exceptionally, and removal is part of the management concept of the national park to allow for a late 
successional stage of purely deciduous forest.

Sampling was carried out in 12 forest stands of different tree diversity and designed a priori to test effects of the tree diversity gradient on beetle diversity. All forest stands shared main characteristics like stagnic luvisol soil on loess-limestone as parent material, flat elevation, absence of canopy gaps, and had remained undisturbed for more than 40 years since the last logging event. To determine tree diversity of each stand, 50 × $50 \mathrm{~m}$ plots were established representing a gradient from mono-dominant beech to mixed forests with up to 11 deciduous tree species. (i.e. Acer platanoides L., Acer pseudoplatanus L., Carpinus betulus L., Fagus sylvatica L., Fraxinus excelsior L., Prunus avium (L.), Quercus robur L., Sorbus torminalis L., Tilia cordata L., Tilia platyphyllos Scop., Ulmus glabra Huds.). We reported tree diversity of the sampled plots as Shannon indices based on stem counts (diameter breast height $>7 \mathrm{~cm}$ ), which accounts for the relative abundance of individual tree species as well as for richness (Magurran 2004) (Appendix 2.1).

\section{Sampling of beetles and sample processing}

Beetles were sampled using cross-window flight interception traps. The traps consisted of two translucent polycarbonate panes measuring $60 \times 40 \mathrm{~cm}$, which were attached to funnels of lorry tarp guiding to an upper and lower collecting jar filled with ethylene-glycol (1:1 diluted with water) as a preserving liquid. Flight-interception traps of this type are non-attractive, and insects are caught by chance when hitting the crossed panes in flight. Across all forest stands, 72 traps (6 traps per stand) were installed in the centre of individual tree crowns using a crossbow and following a sampling scheme based on the relative abundance of beech. In highly beech dominated stands with up to four tree species (83-100\% beech), only beech (Fagus sylvatica) was sampled, in stands with up to seven tree species and at least $48 \%$ beech, the three most dominant species were sampled (beech, lime (Tilia sp.), ash (Fraxinus excelsior), and in stands with up to 11 tree species and the proportion of beech decreasing below $42 \%$, six tree species were sampled (beech, lime, ash, sycamore maple (Acer pseudoplatanus), hornbeam (Carpinus betulus) and either oak (Quercus robur) or service tree (Sorbus torminalis)). Sampled trees were randomly selected on the $50 \times 50 \mathrm{~m}$ plots or in a 10 $\mathrm{m}$ wide corridor in the directly adjacent forest. Clearance of traps was accomplished every four weeks over a period of six months from May to October 2005. To allow for a comparison of single beech trees across the gradient, four additional traps were installed in beech trees on the four most diverse plots. Analyses for beech alone were then based on two randomly drawn or sampled trees per plot. 
Beetles were separated from plant material and other debris and stored in $70 \%$ ethyl alcohol. All individuals were identified to species level. When condition of the material did not allow for species-level determination, specimens were assigned to other taxonomic levels (at least to family). Alcohol-preserved voucher specimens were deposited in an in-house collection (Agroecology, Georg-August-University Göttingen). For each species, information on ecology (rarity, habitat specialisation, feeding guild) was annotated based on details outlined in Böhme (2004) (Appendix 2.2), resulting in the following groupings: common (common or only regionally rare)/rare (rare or very rare), forest species/habitat generalists, and predators/herbivores/fungivores.

\section{Data analyses}

All analyses were performed based on six sampled trees per stand (beech or a mix of species), and for two beech individuals per stand separately. Observed species richness ( $\gamma$-diversity) was calculated as accumulated number of species per plot (pooled over space and time). A non-parametric species estimator, the first order Jackknife, was used to estimate sampling success. The more species appear in a single sampling unit (here tree), the higher the estimated number of species for each plot (Heltshe \& Forrester 1983, Magurran 2004). All plots turned out to be equally sampled (68-75\% of estimated species, Appendix 2.1), hence all subsequent analyses were performed on observed species richness.

Additive partitioning of $\gamma$-diversity was performed for each plot based on Lande (1996), wherein $\gamma$ (overall regional diversity) $=\alpha$ (mean species richness within sample) $+\beta$ (species turnover). Here, we partitioned $\gamma_{\text {stand }}$ in $\alpha+\beta_{\text {time }}+\beta_{\text {space, }}$ with $\alpha$ defined as mean species richness per tree per month, seasonal turnover $\beta_{\text {time }}$ as mean $\beta_{\text {timeTree }}(=$ observed number of species per tree minus $\alpha$ ), and spatial turnover $\beta_{\text {space }}$ specified as observed species richness per plot minus mean number of species per tree (pooled over the sampling season).

Spearman rank correlations were calculated to evaluate the potential of various stand characteristics as explanatory variables for multiple regression analyses and to examine multicollinearity of variables. Tree diversity of the sampled forest stands was highly correlated with several other stand characteristics (Appendix 2.3). To test for linear relationships between tree diversity and the response variables $\left(\gamma\right.$-diversity, $\alpha, \beta_{\text {time, }}, \beta_{\text {space, }}$ factor levels of rarity, habitat specialisation, feeding guild) we used multiple regressions with type I sum of squares with beetle abundance and tree diversity (Shannon index) as explanatory variables. Count data were $\log _{10}$-transformed. Beetle abundance was included in the model to eliminate differences in species richness simply due to differences in individual 
abundance (Magurran 2004). Beetle species richness regressed on beetle abundance accounts for the sampling effect and for the variance in the data explained by abundance (e.g. Knops et al. 1999). This approach is an alternative to rarefaction, and directly relates individual abundance to the actually observed number of species. Rarefaction has recently been criticised as being biased under certain circumstances, thereby leading to questionable results for a correct estimation of species richness (Collins \& Simberloff, in press). Other explanatory variables were not included in the models for reasons of multicollinearity, and because only marginally increased explanatory power was expected based on the multiple $\mathrm{r}^{2}$ values yielded in the simple models including beetle abundance and tree diversity only. Beetle abundance was strongly positively correlated with tree diversity (Pearson's $\rho=0.7, p=0.012$ ), hence we tested different sequences of the variables entered to the model. The latter was not necessary for the communities on beech alone (Pearson's $\rho=0.39, p=0.208$ ). Models were fitted separately for each response variable, and analysis of covariance (ANCOVA) was used to test for differences in regression slopes of $\alpha, \beta_{\text {time }}$ and $\beta_{\text {space-diversity. Model residuals were }}$ examined for meeting assumptions of normality and homoscedasticity.

First order Jackknife estimates were computed with EstimateS 8.0 (Colwell 2006). Other statistical analyses were carried out using R, Version 2.6.2 (R Development Core Team 2007; http://www.R-project.org).

\section{Results}

\section{Species richness of beetles ( $\gamma$-diversity)}

A total of 10,360 individuals belonging to 60 families and 422 species were captured (Appendix 2.2). Observed beetle species richness ( $\gamma$-diversity) showed a highly significant response to the tree diversity gradient after controlling for variance explained by differences in beetle abundance $\left(F_{1,9}=46.44, \mathrm{p}<0.001\right.$, Fig. 2.1$)$. The number of observed beetle species per stand increased from 99 to 181 species with increasing tree diversity. For beech alone, the number of observed beetle species also increased across the tree diversity gradient $\left(F_{1,9}=\right.$ $6.53, \mathrm{p}=0.031)$, but only from 58 to 88 species. 


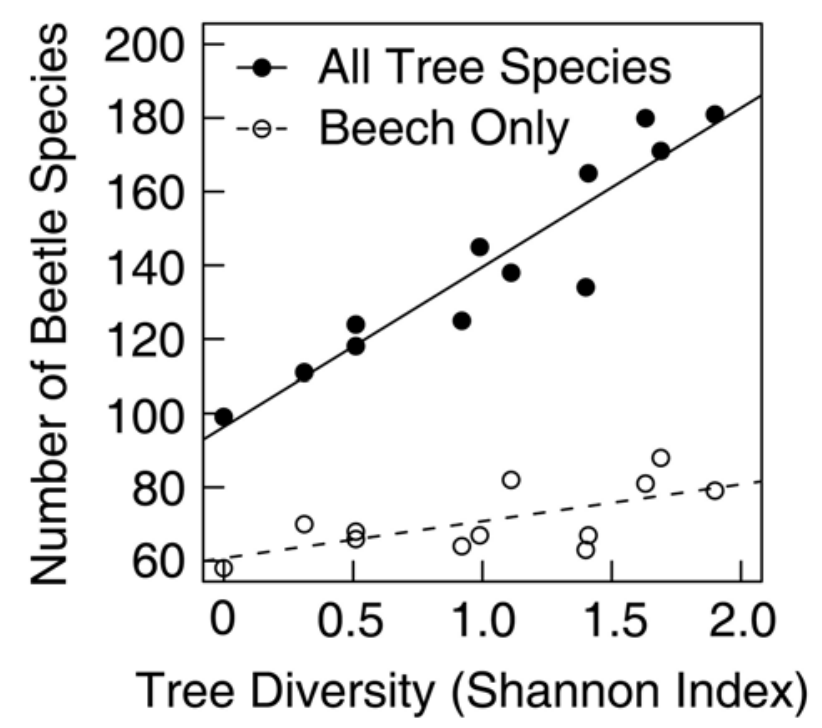

Fig. 2.1. Observed species richness ( $\gamma$-diversity) of beetles across a tree diversity gradient in a Central European forest.

Table 2.1. Multiple regression analyses of species richness parameters for canopy beetles of various tree species across a tree diversity gradient in the Hainich National Park. Abundance= beetle abundance, Shannon Index $=$ tree diversity of the sampled forest stands. ${ }^{\dagger}$ Temporal and spatial species turnover were not linked to turnover in beetle abundance, hence this variable was omitted from the model.

\begin{tabular}{|c|c|c|c|c|c|c|c|}
\hline \multirow[b]{2}{*}{ Response Variable } & \multirow[b]{2}{*}{ Effect } & \multicolumn{3}{|c|}{$\begin{array}{l}\text { Model A: } \\
\text { Abundance + Shannon Index }\end{array}$} & \multicolumn{3}{|c|}{$\begin{array}{l}\text { Model B: } \\
\text { Shannon Index }+ \text { Abundance }\end{array}$} \\
\hline & & Multiple r2 & $F$ & $\mathrm{p}$ & Multiple r2 & $F$ & $\mathrm{p}$ \\
\hline \multirow[t]{2}{*}{ Observed Species Richness } & Abundance & & 171.24 & $<0.001$ & & 18.05 & 0.002 \\
\hline & Shannon Index & 0.96 & 46.44 & $<0.001$ & 0.96 & 199.64 & $<0.001$ \\
\hline \multirow[t]{2}{*}{$\alpha$-Diversity } & Abundance & & 90.32 & $<0.001$ & & 30.13 & $<0.001$ \\
\hline & Shannon Index & 0.91 & 1.04 & 0.336 & 0.91 & 61.23 & $<0.001$ \\
\hline \multirow[t]{2}{*}{$\beta_{\text {time }}$-Diversity } & Abundance $^{\dagger}$ & & & & & & \\
\hline & Shannon Index & 0.77 & 32.76 & $<0.001$ & & & \\
\hline \multirow[t]{2}{*}{$\beta_{\text {space}}$-Diversity } & Abundance $^{\dagger}$ & & & & & & \\
\hline & Shannon Index & 0.89 & 79.33 & $<0.001$ & & & \\
\hline
\end{tabular}


Table 2.2. Multiple regression analyses of species richness parameters for canopy beetles of beech trees across a tree diversity gradient in the Hainich National Park. Abundance= beetle abundance, Shannon Index $=$ tree diversity of the sampled forest stands. ${ }^{\dagger}$ Temporal and spatial species turnover was not linked to turnover in beetle abundance, hence this variable was omitted from the model

\begin{tabular}{lllll}
\hline & & \multicolumn{3}{l}{ Abundance $^{+}$Shannon Index } \\
\hline Response Variable & Effect & Multiple r2 & $F$ & $\mathrm{p}$ \\
\hline Observed Species Richness & Abundance & & 21.04 & $\mathbf{0 . 0 0 1}$ \\
& Shannon Index & 0.75 & 6.53 & $\mathbf{0 . 0 3 1}$ \\
& Abundance & & 16.66 & $\mathbf{0 . 0 0 3}$ \\
& Shiversity & & 0.02 & 0.9 \\
& Shannon Index & 0.65 & & \\
$\beta_{\text {time-Diversity }}$ & Abundance & & & \\
& Shannon Index & 0.11 & 1.19 & 0.3 \\
$\beta_{\text {space-Diversity }}$ & Abundance & & & \\
& Shannon Index & 0.52 & 10.75 & $\mathbf{0 . 0 0 8}$ \\
\hline
\end{tabular}

\section{Additive partitioning of $\gamma$-diversity}

Beetle $\alpha$-diversity responded to the tree diversity gradient only if the Shannon index was introduced to the model first (Shannon: $F_{1,9}=61.32, \mathrm{p}<0.001$, Abundance: $F_{1,9}=90.32, \mathrm{p}<$ 0.001 , Fig. 2.2a). Temporal turnover $\beta_{\text {time }}$ also increased with increasing tree diversity $\left(F_{1,9}=\right.$ $32.76, \mathrm{p}<0.001$, Table 2.1). Spatial turnover $\beta_{\text {space }}$ was higher compared to temporal turnover $\beta_{\text {time }}$ with a steeper slope in the fitted regression (ANCOVA, $F_{2,30}=33.71, \mathrm{p}<0.001$ ), and also showed a highly significant response to increased tree diversity $\left(F_{1,9}=79.33, \mathrm{p}<0.001\right.$, Table 2.1). In contrast, temporal turnover $\beta_{\text {time }}$ was higher if beech alone was analysed (Fig $2.2 \mathrm{~b})$, but in this case did not respond to the tree diversity gradient $\left(F_{1,9}=1.19, \mathrm{p}=0.3\right)$, unlike spatial turnover $\beta_{\text {space }}\left(F_{1,9}=10.75, \mathrm{p}=0.008\right.$, Table 2.2$)$.

There was no proportional change in the contribution of $\alpha$-diversity and temporal or spatial species turnover to overall $\gamma$-diversity within each forest stand across the tree diversity gradient (Appendix 2.4), only relative spatial turnover on beech alone showed a slightly increasing linear relationship $\left(F_{1,9}=5.17, \mathrm{p}=0.049\right)$ 


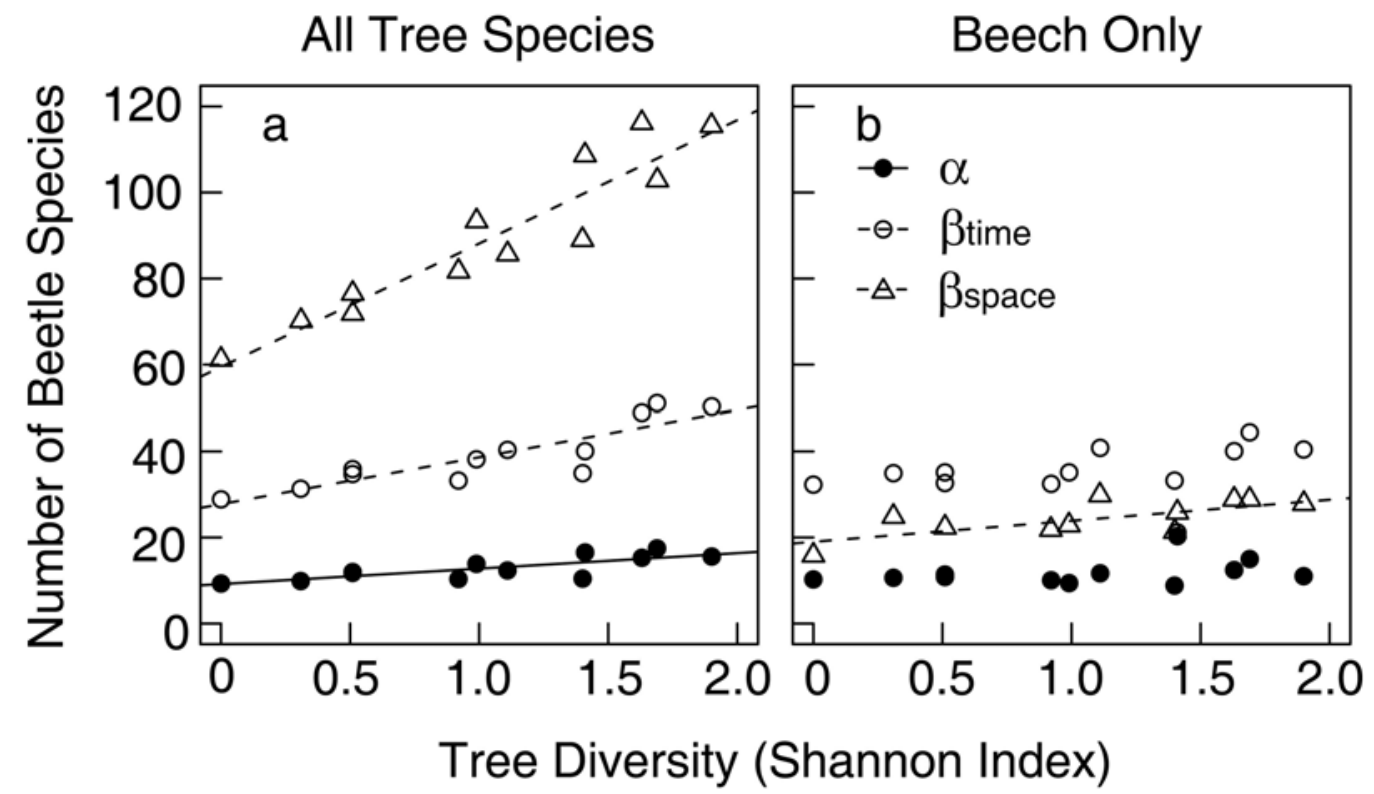

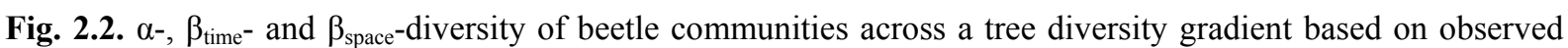
species richness per plot. a) All tree species sampled, regression slopes differ significantly (ANCOVA, $F_{2,30}=$ $33.71, \mathrm{p}<0.001, \mathrm{~b})$ beech only.

\section{Beetle community composition and functional groups}

Most species were classified as habitat generalists (228 species, $54 \%$ ), whereas strictly forest bound species constituted roughly another quarter of the total (115 species, $27.3 \%$, Appendix 2.2). Both groups also constituted the majority of individuals $(7,106$ generalist individuals, $68.6 \% ; 2,405$ forest individuals, $23.3 \%$ ). Less then $1 \%$ of the species were identified as tourists specialised to habitat types other than forest, 67 species $(15.8 \%)$ lacked information on habitat preference. Habitat generalists and forest species both increased in numbers on the most diverse plots (Fig. 2.3a), the linear relationship of forest species to tree diversity was highly significant $\left(F_{1,9}=41.67, \mathrm{p}<0.001\right.$, Table 2.3$)$. The number of forest species also increased linearly if beech alone was compared $\left(F_{1,9}=7.84, \mathrm{p}=0.021\right.$, Fig. 2.3b) while habitat generalists showed no significant response $\left(F_{1,9}=4.77, \mathrm{p}=0.057\right.$, Table 2.4). 
Table 2.3. Multiple regression analyses of functional guild parameters for canopy beetles captured on various tree species across a tree diversity gradient in the Hainich National Park. Abundance $=$ beetle abundance, Shannon Index $=$ tree diversity of the sampled forest stands.

\begin{tabular}{|c|c|c|c|c|c|c|c|}
\hline & & \multicolumn{3}{|l|}{ Model A: } & \multicolumn{2}{|l|}{ Model B: } & Shannon Index + Abundance \\
\hline \multicolumn{8}{|l|}{ Response Variable } \\
\hline Species Richness of & Effect & Multiple r2 & $F$ & $\mathrm{p}$ & Multiple r2 & $F$ & $\mathrm{p}$ \\
\hline \multirow[t]{2}{*}{ Habitat Generalists } & Abundance & & 141.05 & $<0.001$ & & 18.39 & 0.002 \\
\hline & Shannon Index & 0.94 & 9.09 & 0.015 & 0.94 & 131.75 & $<0.001$ \\
\hline \multirow[t]{2}{*}{ Forest Species } & Abundance & & 2.5 & 0.148 & & 5.09 & 0.05 \\
\hline & Shannon Index & 0.83 & 41.67 & $<0.001$ & 0.83 & 39.08 & $<0.001$ \\
\hline \multirow[t]{2}{*}{ Common Species } & Abundance & & 76.03 & $<0.001$ & & 7.96 & 0.02 \\
\hline & Shannon Index & 0.91 & 14.45 & 0.004 & 0.91 & 82.51 & $<0.001$ \\
\hline \multirow[t]{2}{*}{ Rare Species } & Abundance & & 24.37 & $<0.001$ & & 15.07 & 0.004 \\
\hline & Shannon Index & 0.91 & 61.62 & $<0.001$ & 0.91 & 70.93 & $<0.001$ \\
\hline \multirow[t]{2}{*}{ Predators } & Abundance & & 81.77 & $<0.001$ & & 6.1 & 0.036 \\
\hline & Shannon Index & 0.91 & 6.51 & 0.031 & 0.91 & 82.18 & $<0.001$ \\
\hline \multirow[t]{2}{*}{ Herbivores } & Abundance & & 0.62 & 0.452 & & 1.27 & 0.288 \\
\hline & Shannon Index & 0.76 & 28.47 & $<0.001$ & 0.76 & 27.81 & $<0.001$ \\
\hline \multirow[t]{2}{*}{ Fungivores } & Abundance & & 58.35 & $<0.001$ & & 13.12 & 0.006 \\
\hline & Shannon Index & 0.88 & 7.55 & 0.023 & 0.88 & 52.77 & $<0.001$ \\
\hline
\end{tabular}

Based on the classification in Böhme (2004), the majority of species and individuals was denoted as common or only regionally rare (9,093 individuals, $87.8 \% ; 310$ species, $73.5 \%$, Appendix 2.2), 76 species (18\%) as rare or very rare (1,043 individuals, $10 \%)$, whereas for the remaining 36 species ( $8.5 \%$ ) information on rarity was lacking (224 individuals, $2.2 \%$ ). The number of common as well as rare species increased across the tree diversity gradient (Fig. 2.3c), and the response of rare species was highly significant $\left(F_{1,9}=61.62, \mathrm{p}<0.001\right.$, Table 2.3). A similar pattern was found for species on beech alone (Fig. $2.3 \mathrm{~d}$, common: $F_{1,9}=$ $7.66, \mathrm{p}=0.022 ;$ rare: $\left.F_{1,9}=9.65, \mathrm{p}=0.013\right)$. 
Table 2.4. Multiple regression analyses of functional guild parameters for canopy beetles captured on beech trees across a tree diversity gradient in the Hainich National Park. Abundance= beetle abundance, Shannon Index $=$ tree diversity of the sampled forest stands.

\begin{tabular}{lllll}
\hline \multirow{2}{*}{$\begin{array}{l}\text { Response Variable } \\
\text { Species Richness of }\end{array}$} & & \multicolumn{3}{l}{ Abundance + Shannon Index } \\
\cline { 3 - 5 } & & Multiple r2 & $F$ & $\mathrm{p}$ \\
\hline Habitat Generalists & Abundance & 0.77 & 25.96 & $<\mathbf{0 . 0 0 1}$ \\
& Shannon Index & & 4.77 & 0.057 \\
Forest Species & Abundance & 0.61 & 6.33 & $\mathbf{0 . 0 3 3}$ \\
Common Species & Abundance & 0.71 & 14.5 & $\mathbf{0 . 0 0 4}$ \\
& Shannon Index & & 7.66 & $\mathbf{0 . 0 2 2}$ \\
Rare Species & Abundance & 0.77 & 19.89 & $\mathbf{0 . 0 0 2}$ \\
Predators & Shannon Index & & 9.65 & $\mathbf{0 . 0 1 3}$ \\
& Abundance & 0.72 & 12.96 & $\mathbf{0 . 0 0 6}$ \\
Herbivores & Shannon Index & & 9.78 & $\mathbf{0 . 0 1 2}$ \\
& Abundance & 0.5 & 0.35 & 0.57 \\
Fungivores & Shannon Index & & 8.52 & $\mathbf{0 . 0 1 7}$ \\
& Abundance & 0.45 & 4.41 & 0.065 \\
& Shannon Index & & 2.99 & 0.117 \\
\hline
\end{tabular}

Grouped into feeding guilds, more than one third of the species were denoted as predators (36.7\%), followed by 119 herbivore (28.2\%) and 54 fungivore species (12.8\%, Appendix 2.2). Other feeding guilds each did not include more than $5 \%$ of the observed species. In terms of abundance, herbivorous species were most common (3,194 individuals, $30.8 \%)$, whereas one quarter of all individuals was fungivorous (2,782 individuals, $26.9 \%)$ and one quarter predatory (2,588 individuals, $25 \%$ ). Among the herbivorous beetles, 48 species (40.3 $\%$ were identified as wood feeders (1066 individuals, $38.3 \%)$. 
All Tree Species

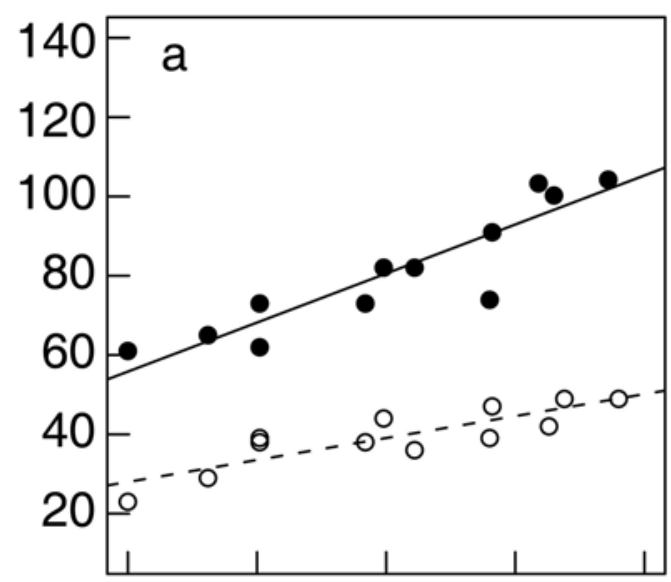

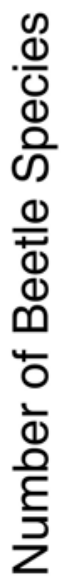
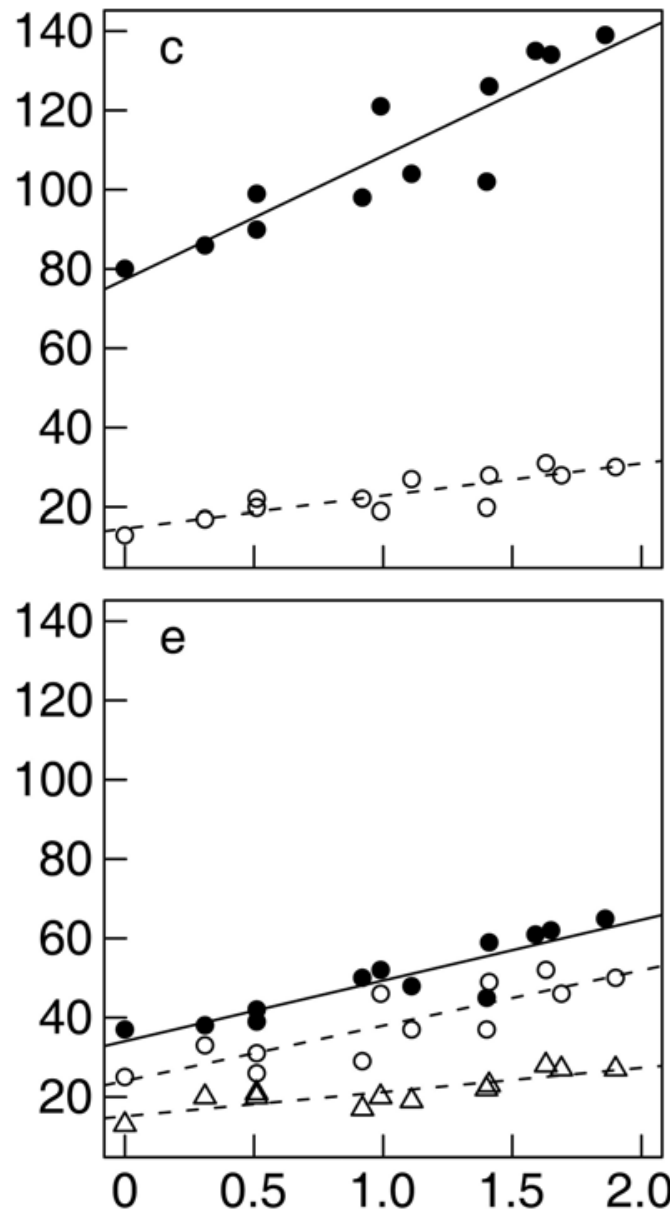

Tree Diversity (Shannon Index)

-A- Fungivores

Beech Only
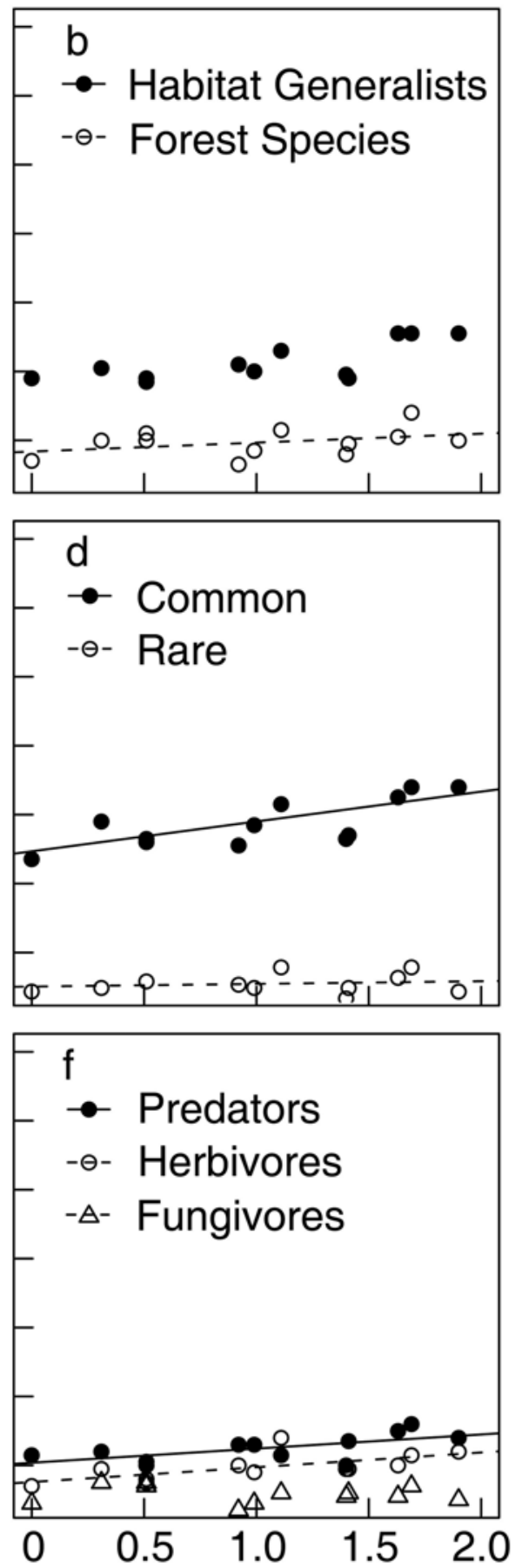
All three guilds showed a positive linear relationship to increased tree diversity (Fig. 2.3 e), with a highly significant response for herbivore species richness $\left(F_{1,9}=58.35, \mathrm{p}<0.001\right.$, Table 2.3), which were not influenced by differences in herbivore abundance across the plots. The number of predatory and herbivorous species also increased across the gradient if beech alone was considered (Fig. 3f, $F_{1,9}=9.78, \mathrm{p}=0.012$ and $F_{1,9}=8.52, \mathrm{p}=0.017$ ), but no effect was found for fungivores $\left(F_{1,9}=2.99, \mathrm{p}=0.117\right)$. No changes in the relative abundance of species in any of the analysed groupings and guilds could be observed within each forest stand across the tree diversity gradient (Appendix 2.5), neither for all tree species nor beech alone.

\section{Discussion}

In agreement with our a priori hypothesis that species-rich forest stands house a greater number of beetle species, overall $\gamma$-diversity of beetles increased across the tree diversity gradient. $\alpha$-diversity was mainly influenced by differences in abundance rather than tree diversity, showing that the mean number of species per tree and month did barely change across plots. In contrast, temporal $\beta$-diversity was higher in the mixed stands, and especially spatial $\beta$-diversity responded strongly to tree diversity indicating a high turnover of beetle species between the tree individuals on the plots. These results show the importance of extensive seasonal and spatial sampling effort for a reliable determination of insect diversity across habitat gradients (Tylianakis et al. 2005). Effective spatial sampling not only demands a sufficient number of sampling replicates (here trees), but also a variety of tree species when forest plots of different diversity are considered. Simply analysing beech alone would have led to the false conclusion that temporal turnover contributes more to beetle diversity than spatial turnover, which is clearly not the case when various tree species or simply more tree individuals are sampled. With an increased number of sampled trees, spatial turnover contributed most to overall beetle diversity. Beech monocultures appear on first sight highly homogeneous, but besides tree species identity as a driver of beetle species richness, individual differences of conspecific trees also seem to play a role for increasing spatial turnover. Summerville et al. (2003b) have shown similar effects for lepidopteran caterpillars, but in contrast to our study, turnover between conspecific trees was generally greater compared to turnover between different tree species. Different insect orders and ontogenetic 
stages thus show different patterns of diversity partitioning, and further research is needed to reveal the mechanisms (e.g. mobility, host preference) behind these patterns.

The observed differences in beetle richness across the a priori defined tree diversity gradient can be explained by changes in habitat heterogeneity, which has been proposed to be the driving force of animal species richness across various ecosystems and taxonomic groups (Tews et al. 2004). Structural parameters like tree species identity, tree dimensions, vertical layering, leaf area index, stand openness, amount of coarse woody debris and deadwood availability have been shown to affect community composition of beetles (Larsson \& Danell 2001, Jukes et al. 2002, Fayt et al. 2006, McGeoch et al. 2007, Müller et al. 2008), and are controlled by canopy tree diversity. In our study, stand structure and complexity changed with the mix and altered biotic and abiotic conditions; the highly diverse plots sampled in this study showed a higher abundance and species richness of herbaceous plant species in the understory (Mölder et al. 2006). Furthermore, different architecture of various tree species itself is an important factor determining niche-availability and the diversity of associated arthropod communities by controlling micro-climate, shelter-availability and accessibility (Lawton 1983, Southwood et al. 1982, Halaj et al. 1998, Halaj et al. 2000, Goßner \& Ammer 2006). Compared to mono-dominant beech stands, diverse forest stands thus offer a greater array of niches and resources, which enhances beetle diversity.

Alternative hypotheses to explain differences in forest beetle diversity are previous or current human disturbance (Nilsson \& Baranowski 1997, Goßner et al. 2006) and stand age (Hammond et al. 2004, Grimbacher \& Catterall 2007) or stand productivity, which are negligible in our case. None of the investigated stands was pristine, and stand age (I. Schmidt, pers. com.) as well as productivity (Jacob et al., submitted) decreased with increased tree diversity, with the youngest stands exhibiting the highest tree and beetle diversity. The latter is particularly striking, because old-age of forest stands is commonly used to explain high beetle diversity. Our results illustrate that apparently even old-age in mono-dominant beech stands does not increase beetle species richness compared to considerably younger forest stands with a diverse mix of tree species. Nevertheless, it should be acknowledged that human impact might be a reason for the overall lack of old-growth relict beetle species sensu Müller et al. (2005) (Grossmann 2006).

In total, one third of the sampled beetle species was classified as habitat specialists, a proportion strikingly consistent with results from other studies in European forest and grassland habitats (Magura et al. 2001, Batary et al. 2007). As expected, more rare species and forest specialists were captured in the most diverse forest stands, but also common and 
generalist species preferred the more heterogenous mixed forests. This is probably due to a general lack of available resources in simpler habitats (Gotelli \& Colwell 2001), which was also reflected by lower individual abundance in the less diverse beech stands. Increased species richness within a sampled stand also resulted in increased richness within functional groups. Here, predators outnumbered phytophageous and mycetophageous species across all forest types, and all guilds increased in species richness across the tree diversity gradient, so that the relative proportions within each plot remained constant. This outcome appears to be typical for forest habitats, and different tree species have shown to be consistent by means of relative abundance of feeding guilds (Moran \& Southwood 1982, Southwood et al. 1982, Jukes et al. 2002). Predators tended to be the most speciose guild in various studies, a pattern that seems to be uniform throughout different climate zones and vastly independent of stand structure and vegetation diversity (Southwood et al. 1982, Jukes et al. 2002).

We conclude that for a sufficient judgement of arthropod biodiversity patterns in forest ecosystems, it is crucial to include a variety of spatiotemporal scales in the analyses (Gering $\&$ Crist 2000). It was clearly demonstrated by our comparison of beech alone in contrast to various tree species, that otherwise observations can easily result in misleading conclusions, if for example only certain plant species, time points or not enough individuals are considered. Especially the inclusion of temporal and spatial turnover ( $\beta$-diversity) adds value to the analyses (Gering et al. 2007), and allows for a fine- grained evaluation of how diversity patterns evolve and what contributes most to the total diversity observed in a region, which in our case was turnover $\left(\beta_{\text {space }}\right)$ of beetles among trees.

Furthermore, it was evident that mono-dominant beech stands alone are unsatisfactory in conserving the full set of regional beetle species richness, as opposed to findings by Gering et al. (2003), who recommend that rather than maintaining high local tree diversity, establishment of multiple forest sites within ecoregions is of importance. We suggest that sustainable forest management should also aim for maintaining a diverse mix of structurally different tree species, thus enhancing spatial heterogeneity, habitat complexity, and providing resources for a diverse beetle community within sites. Intensive forestry leads to habitat loss, fragmentation and reduced complexity (Kouki et al. 2001, Larsson \& Danell 2001, Hirao et al. 2007), which will eventually result in a scattered, over-simplified forest landscape not suitable to preserve high beetle diversity. Hence, we recommend an integrative approach of forest management by increasing tree diversity locally and site diversity regionally. Species rich forests not only play an important role in conserving rare and strictly forest dependent species, but also house the majority of generalist species present in a certain area. Failing to 
include these species in future conservation planning might result in the decline of local populations, and finally lead to a depletion of regional species richness. Due to their abundance, these species might also be functionally dominant (Summerville et al. 2003a), and thus of relevance for maintaining community interactions and forest ecosystem functions (Gering et al. 2003). To reveal hidden differences and driving forces of diversity and community patterns, we moreover recommend that examinations of insect communities should use habitat gradients at different spatiotemporal scales instand of pairwise comparisons of i. e. managed/unmanaged stands, which currently dominate the available literature.

\section{Acknowledgements}

This study obtained financial support by the DFG [German Research Foundation] within the Research Training Group „Graduiertenkolleg 1086: The role of biodiversity for biogeochemical cycles and biotic interactions in temperate deciduous forests“. We highly acknowledge Christoph Leuschner, Frank Thomas, Hermann Jungkunst and Stefan Fleck for coordination of the group and also thank Mascha Jacob, Karl M. Daenner, Mareen Gollnow, Inga Krämer and Inga Schmidt for providing data on stand characteristics, furthermore Boris Büche for his support with beetle identification. Our special thanks go to Martin Erren, Tobias Gebauer, Martin Goßner, Susanne Schiele and Ulrich Simon for assistance with field work. We also thank Thomas Crist and two anonymous reviewers for helpful comments to improve the manuscript and Randy Letkeman for proof-reading. 


\section{References}

Batary, P., Baldi, A., Szel, G., Podlussany, A., Rozner, I., \& Erdos, S. (2007) Responses of Grassland Specialist and Generalist Beetles to Management and Landscape Complexity. Diversity and Distributions 13, 196-202.

Böhme, J. (2004) Die Käfer Mitteleuropas: Katalog (Faunistische Übersicht), Band K Spektrum Akademischer Verlag, Heidelberg.

Collins, M.D. \& Simberloff, M (in press) Rarefaction and Nonrandom Dispersion Patterns. Environmental and Ecological Statistics. DOI 10.1007/s10651-007-0051-y

Colwell, R.K. (2006) EstimateS: Statistical Estimation of Species Richness and Shared Species from Samples. Version 7.5. User's guide and application published at http://purl.oclc.org/estimates.

Crist, T. O., Veech, J. A., Gering, J. C., \&Summerville, K. S. (2003) Partitioning Species Diversity Across Landscapes and Regions: a Hierarchical Analysis of Alpha, Beta, and Gamma Diversity. American Naturalist 162, 734-743.

Davies, Z.G., Tyler, C., Stewart, G.B., \& Pullin, A.S. (2008) Are Current Management Recommendations for Saproxylic Invertebrates Effective? A Systematic Review. Biodiversity and Conservation 17, 209-234.

Ellenberg, H. (1996) Vegetation Mitteleuropas mit den Alpen in ökologischer, dynamischer und historischer Sicht. Ulmer, Stuttgart.

Erwin, T.L. (1982) Tropical Forests: Their Richness in Coleoptera and Other Arthropod Species. The Coleopterist's Bulletin 36, 74-75.

Erwin, T.L. (1988) The Tropical Forest Canopy- The Heart of Biotic Diversity. Biodiversity (ed. by E.O. Wilson), National Academy Press.

Erwin, T.L. (1997) Biodiversity at Its Utmost: Tropical Forest Beetles . Biodiversity II: Understanding and Protecting Our Biological Resources (ed. by M.J. Reaka-Kudla, D.E. Wilson, and E.O. Wilson), Joseph Henry Press.

Fayt, P., Dufrene, M., Branquart, E., Hastir, P., Pontegnie, C., Henin, J.M., \& Versteirt, V. 
(2006) Contrasting Responses of Saproxylic Insects to Focal Habitat Resources: the Example of Longhorn Beetles and Hoverflies in Belgian Deciduous Forests. Journal of Insect Conservation 10, 129-150.

Gaylord, M.L., Kolb, T.E, Wallin, K.F., \& M.R. Wagner (2006) Seasonality and Lure Preference of Bark Beetles (Curculionidae: Scolytinae) and Associates in a Northern Arizona Ponderosa Pine Forest. Environmental Entomology 35, 37-47.

Gering, J.C. \& Crist, T.O. (2000) Patterns of Beetle (Coleoptera) Diversity in Crowns of Representative Tree Species in an Old-Growth Temperat Deciduos Forest. Selbyana 21, 3847.

Gering, J.C. \& Crist, T.O. (2002) The Alpha-Beta-Regional Relationship: Providing New Insights into Local-Regional Patterns of Species Richness and Scale Dependence of Diversity Components. Ecology Letters 5, 433-444.

Gering, J.C., Crist, T.O. \& Veech, J.A. (2003) Additive partitioning of beetle species diversity across multiple spatial scales: implications for regional conservation of biodiversity. Conservation Biology 17, 488-499.

Gering, J.C., DeRennaux, K.A., \& Crist, T.O. (2007) Scale dependence of effective specialisation: its analysis and implications for estimates of global insect species richness. Diversity and Distributions 13, 115-125.

Gessler, A., Schneider, S., Weber, P., Hanemann, U., \& Rennenberg, H. (1998) Soluble N Compounds in Trees Exposed to High Loads of N: a Comparison Between the Roots of Norway Spruce (Picea abies) and Beech (Fagus sylvatica) Trees Grown Under Field Conditions. New Phytologist 138, 385-399.

Goßner, M. \& Ammer, U. (2006) The Effects of Douglas-Fir on Tree-Specific Arthropod Communities in Mixed Species Stands With European Beech and Norway Spruce. European Journal of Forest Research 125, 221-235.

Goßner, M., Engel, K., \& Ammer, U. (2006) Effects of Selection Felling and Gap Felling on Forest Arthropod Communities: a Case Study in a Spruce-Beech Stand of Southern Bavaria. European Journal of Forest Research 125, 345-360.

Gotelli, N.J. \& Colwell, R.K. (2001) Quantifying Biodiversity: Procedures and Pitfalls in the 
Measurement and Comparison of Species Richness. Ecology Letters 4, 379-391.

Grimbacher, P.S. \& Catterall, C.P. (2007) How Much Do Site Age, Habitat Structure and Spatial Isolation Influence the Restoration of Rainforest Beetle Species Assemblages? Biological Conservation 135, 107-118.

Grossmann, M. (2006) Forschung im Nationalpark Hainich/Thüringen. Waldökologie online $3,63-66$

Grove, S.J. (2002) Saproxylic Insect Ecology and the Sustainable Management of Forests. Annual Review of Ecology and Systematics 33, 1-23.

Grove, S.J. \& Stork, N.E. (2000) An Inordinate Fondness for Beetles. Invertebrate Taxonomy 14, 733-739.

Halaj, J., Ross, D.W., \& Moldenke, A.R. (1998) Habitat Structure and Prey Availability as Predictors of the Abundance and Community Organisation of Spiders in Western Oregon Forest Canopies. Journal of Arachnology 26, 203-220.

Halaj, J., Ross, D.W., \& Moldenke, A.R. (2000) Importance of Habitat Structure to the Arthropod Food-Web in Douglas-Fir Canopies. Oikos 90, 139-152.

Hammond, H.E.J., Langor, D.W., \& Spence, J.R. (2004) Saproxylic Beetles (Coleoptera) Using Populus in Boreal Aspen Stands of Western Canada: Spatiotemporal Variation and Conservation of Assemblages. Canadian Journal of Forest Research-Revue Canadienne De Recherche Forestiere 34, 1-19.

Heltshe, J.F. \& Forrester, N.E. (1983) Estimating Species Richness using the Jacknife Procedure. Biometrics 39, 1-11.

Hirao, T., Murakami, M., Kashizaki, A., \& Tanabe, S.I. (2007) Additive Apportioning of Lepidopteran and Coleopteran Species Diversity Across Spatial and Temporal Scales in a Cool-Temperate Deciduous Forest in Japan. Ecological Entomology 32, 627-636.

Hulcr, J., Mogia, M., Isua, B., \& Novotny, V. (2007) Host Specificity of Ambrosia and Bark Beetles (Col., Curculionidae : Scolytinae and Platypodinae) in a New Guinea Rainforest. Ecological Entomology 32, 762-772.

Huston, M.A. (1999) Local Processes and Regional Patterns: Appropriate Scales for 
Understanding Variation in the Diversity of Plants and Animals. Oikos 86, 393-401.

Jacob, M., Leuschner, C., \& Thomas, F. (submitted) Productivity of a Naturally Predominant Temperat Tree Species Along a Gradient of Increasing Tree Species Diversity.

Jukes, M.R., Ferris, R., \& Peace, A.J. (2002) The Influence of Stand Structure and Composition on Diversity of Canopy Coleoptera in Coniferous Plantations in Britain. Forest Ecology and Management 163, 27-41.

Kenderes, K., Mihos, B., \& Standovar, T. (2008) Thirty Years of Gap Dynamics in a Central European Beech Forest Reserve. Forestry 81, 111-123.

Kouki, J., Lofman, S., Martikainen, P., Rouvinen, S., \& Uotila, A. (2001) Forest Fragmentation in Fennoscandia: Linking Habitat Requirements of Wood-Associated Threatened Species to Landscape and Habitat Changes. Scandinavian Journal of Forest Research 16, 27-37.

Knops, J.M.H., Tilman, D., \& Haddad, N.M. (1999) Effects of Plant Species Richness on Invasion Dynamics, Disease Outbreaks, Insect Abundances and Diversity. Ecology Letters 2, 286-293.

Lande, R. (1996) Statistics and Partitioning of Species Diversity, and Similarity Among Multiple Communities. Oikos 76, 5-13.

Larsson, S. \& Danell, K. (2001) Science and the Management of Boreal Forest Biodiversity. Scandinavian Journal of Forest Research, Supplement 3, 5-9.

Lassau, S.A., Hochuli, D.F., Cassis, G., \& Reid, C.A.M. (2005) Effects of Habitat Complexity on Forest Beetle Diversity: Do Functional Groups Respond Consistently? Diversity and Distributions 11, 73-82.

Lawton, J.H. (1983) Plant Architecture and the Diversity of Phytophagous Insects. Annual Review of Entomology 28, 23-29.

Magura, T., Tothmeresz, B., \& Molnar, T. (2001) Forest Edge and Diversity: Carabids Along Forest-Grassland Transects. Biodiversity and Conservation 10, 287-300.

Magurran, A.E. (2004) Measuring Biological Diversity. Blackwell, Oxford. 
McGeoch, M.A., Schroeder, M., Ekbom, B., \& Larsson, S. (2007) Saproxylic Beetle Diversity in a Managed Boreal Forest: Importance of Stand Characteristics and Forestry Conservation Measures. Diversity and Distributions 13, 418-429.

May, R.M. (1990) How many species? Philosophical Transactions of the Royal Society of London Series B, Biological Sciences 330, 293-304.

Mölder, A., Bernhardt-Römermann, M., \& Schmidt, W. (2006) Forest Ecosystem Research in Hainich National Park (Thuringia): First results on flora and vegetation in stands with contrasting tree species diversity. Waldökologie online 3, 83-99.

Mölder, A., Bernhardt-Römermann, M., \& Schmidt, W. (2008) Herb-Layer Diversity in Deciduous Forests: Raised by Tree Richness or Beaten by Beech? Forest Ecology and Management 256, 272-281.

Moran, V.C. \& Southwood, T.R.E. (1982) The Guild Composition of Arthropod Communities in Trees. Journal of Animal Ecology 51, 289-306.

Müller, J., Bussler, H., Bense, U., Brustel, H., Flechtner, G., Fowles, A., Kahlen, M., , M.G., Mühle, H., Schmidl, J., \& Zabransky, P. (2005) Urwald Relict Species- Saproxylic Beetles Indicating Structural Qualities and Habitat Tradition. Waldökologie online 2, 106-113.

Müller, J., Bussler, H., \& Kneib, T. (2008) Saproxylic Beetle Assemblages Related to Silvicultural Management Intensity and Stand Structures in a Beech Forest in Southern Germany. Journal of Insect Conservation 12, 107-124.

Müller, J. \& Goßner, M. (2007) Single Host Trees in a Closed Forest Canopy Matrix: a Highly Fragmented Landscape? Journal of Applied Entomology 131, 613-620.

Nilsson, S.G. \& Baranowski, R. (1997) Habitat Predictability and the Occurrence of Wood Beetles in Old-Growth Beech Forests. Ecography 20, 491-498.

Novotny, V. \& Basset, Y. (2005) Review - Host Specificity of Insect Herbivores in Tropical Forests. Proceedings of the Royal Society B-Biological Sciences 272, 1083-1090.

Reeve, J.D. (1997) Predation and Bark Beetle Dynamics. Oecologia 112, 48-54.

Southwood, T.R.E., Moran, V.C., \& Kennedy, C.E.J. (1982) The Richness, Abundance and Biomass of the Arthropod Communities on Trees. Journal of Animal Ecology 51, 635-649. 
Stork, N.E. (1988) Insect diversity: facts, fiction and speculation. Biological Journal of the Linnean Society 35, 321-337.

Summerville, K.S, Boulware, M.J., Veech, J.A., \& Crist, T.O. (2003a) Spatial Variation in Species Diversity and Composition of Forest Lepidoptera in Eastern Deciduos Forests of North America. Conservation Biology 17, 1045-1057.

Summerville, K.S., Crist, T.O., Kahn, J.K., \& Gering, J.C. (2003b) Community Structure of Arboreal Caterpillars Within and Among Four Tree Species of the Eastern Deciduous Forest. Ecological Entomology 28, 747-757.

Tews, J., Brose, U., Grimm, V., Tielborger, K., Wichmann, M.C., Schwager, M., \& Jeltsch, F. (2004) Animal Species Diversity Driven by Habitat Heterogeneity/Diversity: the Importance of Keystone Structures. Journal of Biogeography 31, 79-92.

Tylianakis, J.M., Klein, A.M., \& Tscharntke, T. (2005) Spatiotemporal Variation in the Diversity of Hymenoptera Across a Tropical Habitat Gradient. Ecology 86, 3296-3302.

Ulyshen, M.D. \& Hanula, J.L. (2007) A Comparison of the Beetle (Coleoptera) Fauna Captured at Two Heights Above the Ground in a North American Temperate Deciduous Forest. American Midland Naturalist 158, 260-278.

Veech, J.A., Summerville, K. S., Crist, T.O., \& Gering, J.C. (2002) The Additive Partitioning of Species Diversity: Recent Revival of an Old Idea. Oikos 99, 3-9.

Vehviläinen, H., Koricheva, J., \& Ruohomäki, K. (2008) Effects of Stand Tree Species Composition and Diversity on Abundance of Predatory Arthropods. Oikos 117, 935-943.

Wagner, T. (2000) Influence of Forest Type and Tree Species on Canopy-Dwelling Beetles in Budongo Forest, Uganda. Biotropica 32, 502-514. 


\section{Appendix 2.1}

Tree diversity and sampling success of beetle communites in 12 deciduous forest stands in the Hainich National Park. Stands were ranked based on increasing Shannon Indices (tree diversity), rank 3 was assigned twice due to concording Shannon indices.

\begin{tabular}{lllll}
\hline Shannon Index & Stand Ranking & \% Beech & \% Lime & \% Sampling Succes \\
\hline 0 & 1 & 100 & 0 & 72.1 \\
0.31 & 2 & 93.5 & 2.8 & 70.4 \\
0.51 & $3 \mathrm{a}$ & 83.3 & 0 & 71.3 \\
0.51 & $3 \mathrm{~b}$ & 87.5 & 3.6 & 70.6 \\
0.92 & 4 & 73.7 & 10.8 & 69 \\
0.99 & 5 & 59.4 & 2.3 & 71.2 \\
1.11 & 6 & 60.6 & 12.1 & 74.7 \\
1.4 & 7 & 47.7 & 33 & 69.7 \\
1.41 & 8 & 41.9 & 34.2 & 67.8 \\
1.63 & 9 & 2.4 & 63.9 & 70.1 \\
1.69 & 10 & 3.1 & 67.4 & 72 \\
1.9 & 11 & 13.2 & 37.9 & 70 \\
\hline
\end{tabular}




\section{Appendix 2.2}

List of beetle species collected in the canopy of forest stands across a tree diversity gradient in the Hainich National Park. Annotations to habitat, feeding guild, food preference and rarity after Böhme (2004). $n^{v}=$ number of beetles caught on various tree species, $\mathrm{n}^{\mathrm{b}}=$ number of beetles captured on beech alone.

\begin{tabular}{|c|c|c|c|c|c|c|}
\hline Family & Species & Habitat & Feeding Guild & Rarity & $\mathrm{n}^{\mathrm{v}}$ & $\mathrm{n}^{\mathrm{b}}$ \\
\hline Aderidae & Euglenes oculatus & forest & other & rare & 3 & 0 \\
\hline \multirow[t]{4}{*}{ Alleculidae } & Allecula morio & forest & other & common & 11 & 2 \\
\hline & Mycetochara flavipes & forest & other & rare & 2 & 1 \\
\hline & Mycetochara linearis & forest & other & common & 24 & 2 \\
\hline & Prionychus ater & forest & other & common & 1 & 0 \\
\hline \multirow[t]{9}{*}{ Anobiidae } & Dorcatoma chrysomelina & forest & fungivore & rare & 2 & 0 \\
\hline & Dryophilus pusillus & other & herbivore & common & 2 & 1 \\
\hline & Ernobius mollis & forest & herbivore & common & 1 & 0 \\
\hline & Hemicoelus costatus & no preference & herbivore & common & 79 & 31 \\
\hline & Hemicoelus fulvicornis & no preference & herbivore & common & 2 & 0 \\
\hline & Hemicoelus nitidum & no preference & herbivore & common & 8 & 0 \\
\hline & Ptilinus pectinicornis & forest & herbivore & common & 1 & 0 \\
\hline & Ptinomorphus imperalis & other & herbivore & common & 116 & 37 \\
\hline & Xestobium plumbeum & forest & herbivore & common & 39 & 8 \\
\hline \multirow[t]{4}{*}{ Anthribidae } & Brachytarsus nebulosus & no preference & predator & common & 4 & 1 \\
\hline & Choragus sheppardi & forest & fungivore & rare & 1 & 1 \\
\hline & Enedreutes sepicola & unknown & fungivore & rare & 1 & 0 \\
\hline & Tropideres albirostris & forest & fungivore & common & 1 & 0 \\
\hline \multirow[t]{3}{*}{ Apionidae } & Holotrichapion pisi & other & herbivore & common & 1 & 0 \\
\hline & Ischnopterapion virens & no preference & herbivore & common & 2 & 1 \\
\hline & Protapion fulvipes & no preference & herbivore & common & 1 & 0 \\
\hline \multirow[t]{2}{*}{ Bruchidae } & Bruchidius varius & other & herbivore & rare & 1 & 0 \\
\hline & Bruchus rufimanus & no preference & herbivore & rare & 5 & 1 \\
\hline Byturidae & Byturus tomentosus & no preference & herbivore & common & 5 & 1 \\
\hline \multirow[t]{19}{*}{ Cantharidae } & Absindia rufotestacea & no preference & predator & common & 3 & 0 \\
\hline & Cantharis decipiens & no preference & predator & common & 59 & 5 \\
\hline & Cantharis nigricans & no preference & predator & common & 11 & 2 \\
\hline & Cantharis pellucida & no preference & predator & common & 8 & 3 \\
\hline & Cantharis rufa & no preference & predator & common & 1 & 0 \\
\hline & Lagria hirta & no preference & herbivore & common & 2 & 0 \\
\hline & Malthinus sp. & unknown & predator & unknown & 1 & 0 \\
\hline & Malthodes guttifer & no preference & predator & common & 1 & 0 \\
\hline & Malthodes marginatus & no preference & predator & rare & 1 & 0 \\
\hline & Malthodes minimus & no preference & predator & common & 6 & 1 \\
\hline & Malthodes minutus & unknown & unknown & unknown & 2 & 1 \\
\hline & Malthodes pumilus & unknown & predator & rare & 2 & 0 \\
\hline & Malthodes sp. & unknown & predator & unknown & 7 & 0 \\
\hline & Malthodes spathifer & no preference & predator & common & 84 & 9 \\
\hline & Podabrus alpinus & no preference & predator & common & 1 & 0 \\
\hline & Rhagonycha fulva & no preference & predator & common & 8 & 2 \\
\hline & Rhagonycha lignosa & no preference & predator & common & 79 & 14 \\
\hline & Rhagonycha lutea & no preference & predator & common & 5 & 0 \\
\hline & Rhagonycha translucida & no preference & predator & rare & 9 & 3 \\
\hline
\end{tabular}




\begin{tabular}{|c|c|c|c|c|c|}
\hline Carabidae & Agonum muelleri & no preference & predator & common & 2 \\
\hline & Amara communis & other & other & common & 1 \\
\hline & Amara familiaris & no preference & other & common & 3 \\
\hline & Amara lunicollis & no preference & other & common & 1 \\
\hline & Amara montivaga & unknown & other & common & 2 \\
\hline & Amara plebeja & unknown & other & common & 1 \\
\hline & Amara similata & unknown & herbivore & common & 5 \\
\hline & Bembidion quadrimaculatum & unknown & predator & common & 1 \\
\hline & Bradycellus verbasci & unknown & other & common & 1 \\
\hline & Calodromius spilotus & forest & predator & common & 1 \\
\hline & Calosoma inquisitor & forest & predator & common & 1 \\
\hline & Dromius agilis & forest & predator & common & 8 \\
\hline & Dromius fenestratus & forest & predator & common & 1 \\
\hline & Dromius quadrimaculatus & forest & predator & common & 168 \\
\hline & Dromius sp. & unknown & predator & unknown & 1 \\
\hline & Harpalus affinis & no preference & other & common & 1 \\
\hline & Limodromus assimilis & no preference & predator & common & 1 \\
\hline & Loricera pilicornis & no preference & predator & common & 8 \\
\hline & Microlestes minutulus & unknown & predator & common & 1 \\
\hline & Notiophilus biguttatus & no preference & predator & common & 3 \\
\hline & Platynus dorsalis & unknown & unknown & unknown & 1 \\
\hline & Pterostichus oblongopunctatus & forest & predator & common & 1 \\
\hline & Trechus quadristriatus & no preference & predator & common & 53 \\
\hline Cerambycidae & Alosterna tabacicolor & forest & herbivore & common & 11 \\
\hline & Anaglyptus mysticus & forest & herbivore & rare & 0 \\
\hline & Grammoptera abdominalis & forest & herbivore & rare & 1 \\
\hline & Grammoptera ruficornis & forest & herbivore & common & 5 \\
\hline & Leiopus nebulosus & no preference & herbivore & common & 17 \\
\hline & Mesosa nebulosa & no preference & herbivore & common & 1 \\
\hline & Oberea linearis & no preference & herbivore & rare & 0 \\
\hline & Phymatodes testaceus & no preference & herbivore & common & 1 \\
\hline & Pogonocherus hispidus & no preference & herbivore & common & 2 \\
\hline & Rhagium mordax & forest & herbivore & common & 7 \\
\hline & Saperda scalaris & no preference & herbivore & common & 1 \\
\hline & Stenocorus meridianus & no preference & herbivore & common & 1 \\
\hline & Stenostola dubia & no preference & herbivore & common & 15 \\
\hline & Tetropium castaneum & forest & herbivore & common & 1 \\
\hline & Tetrops starkii & forest & herbivore & rare & 9 \\
\hline Cerylonidae & Cerylon ferrugineum & forest & other & common & 28 \\
\hline & Cerylon histeroides & no preference & other & common & 1 \\
\hline Cholevidae & Nargus velox & forest & other & common & 1 \\
\hline & Sciodrepoides watsoni & no preference & other & common & 4 \\
\hline Chrysomelidae & Aphthona euphorbiae & unknown & herbivore & rare & 54 \\
\hline & Aphthona venustula & no preference & herbivore & common & 1 \\
\hline & Chaetocnema concinna & no preference & herbivore & common & 0 \\
\hline & Cryptocephalus frontalis & unknown & herbivore & rare & 2 \\
\hline & Galeruca poтопае & no preference & herbivore & common & 1 \\
\hline & Galeruca tanaceti & no preference & herbivore & common & 2 \\
\hline & Longitarsus kutscherae & no preference & herbivore & rare & 2 \\
\hline & Longitarsus luridus & no preference & herbivore & common & 8 \\
\hline & Longitarsus parvulus & no preference & herbivore & common & 17 \\
\hline
\end{tabular}




\begin{tabular}{|c|c|c|c|c|c|}
\hline & Orsodacne cerasi & no preference & herbivore & common & 46 \\
\hline & Oulema gallaeciana & other & herbivore & common & 254 \\
\hline & Oulema melanopus & no preference & herbivore & common & 14 \\
\hline & Phyllotreta atra & no preference & herbivore & common & 6 \\
\hline & Phyllotreta nigripes & no preference & herbivore & common & 5 \\
\hline & Phyllotreta vittula & no preference & herbivore & common & 6 \\
\hline Cisidae & Cis bidentatus & forest & fungivore & rare & 1 \\
\hline & Cis boleti & no preference & fungivore & common & 1 \\
\hline & Ennearthron cornutum & no preference & fungivore & common & 3 \\
\hline & Orthocis alni & no preference & fungivore & common & 6 \\
\hline Clambidae & Clambus sp. & unknown & fungivore & unknown & 2 \\
\hline Cleridae & Opilo mollis & forest & predator & common & 25 \\
\hline & Tillus elongatus & no preference & predator & common & 1 \\
\hline Coccinellidae & Adalia decempunctata & no preference & predator & common & 17 \\
\hline & Calvia decemguttata & no preference & predator & rare & 1 \\
\hline & Chilocorus renipustulatus & no preference & predator & common & 5 \\
\hline & Coccinella septempunctata & no preference & predator & common & 5 \\
\hline & Exochomus quadripustulatus & no preference & predator & common & 17 \\
\hline & Halyzia sedecimguttata & forest & fungivore & common & 9 \\
\hline & Propylea quatuordecimpunctata & no preference & predator & common & 11 \\
\hline & Scymnus abietis & no preference & predator & common & 3 \\
\hline Colydiidae & Synchita humeralis & forest & fungivore & common & 2 \\
\hline Corylophidae & Orthoperus sp. & forest & predator & unknown & 3 \\
\hline & Sacium pusillum & no preference & predator & rare & 13 \\
\hline & Sericoderus lateralis & other & predator & common & 1 \\
\hline Cryptophagidae & Atomaria analis & no preference & other & common & 43 \\
\hline & Atomaria atricapilla & no preference & other & common & 267 \\
\hline & Atomaria fuscata & no preference & other & common & 6 \\
\hline & Atomaria lewisi & no preference & other & common & 2 \\
\hline & Atomaria linearis & no preference & other & common & 124 \\
\hline & Atomaria nigrirostris & no preference & other & common & 2 \\
\hline & Atomaria puncticollis & no preference & other & rare & 1 \\
\hline & Atomaria punctithorax & no preference & other & rare & 7 \\
\hline & Atomaria pusilla & no preference & other & common & 1 \\
\hline & Atomaria sp. & unknown & other & unknown & 9 \\
\hline & Atomaria testacea & no preference & other & common & 13 \\
\hline & Cryptophagus acutangulus & no preference & other & common & 1 \\
\hline & Cryptophagus dentatus & no preference & other & common & 37 \\
\hline & Cryptophagus intermedius & unknown & other & rare & 2 \\
\hline & Cryptophagus pilosus & no preference & other & common & 23 \\
\hline & Cryptophagus pubescens & no preference & other & common & 3 \\
\hline & Cryptophagus scanicus & no preference & other & common & 8 \\
\hline & Cryptophagus sp. & unknown & other & unknown & 1 \\
\hline & Micrambe abietis & forest & fungivore & common & 2 \\
\hline Cucujidae & Phloeostichus denticollis & unknown & unknown & rare & 8 \\
\hline Curculionidae & Ceutorhynchus floralis & no preference & herbivore & common & 1 \\
\hline & C.pallidactylus & no preference & herbivore & common & 8 \\
\hline & Curculio glandium & no preference & herbivore & common & 11 \\
\hline & Curculio pellitus & other & herbivore & rare & 1 \\
\hline & Curculio pyrrhoceras & other & herbivore & common & 2 \\
\hline & Curculio villosus & other & herbivore & common & 1 \\
\hline
\end{tabular}




\begin{tabular}{|c|c|c|c|c|c|c|}
\hline & Furcipus rectirostris & no preference & herbivore & common & 2 & 3 \\
\hline & Hypera venusta & other & herbivore & common & 2 & 0 \\
\hline & Larinus planus & unknown & unknown & unknown & 1 & 1 \\
\hline & Magdalis armigera & forest & herbivore & common & 1 & 0 \\
\hline & Magdalis flavicornis & no preference & herbivore & common & 1 & 0 \\
\hline & Phyllobius argentatus & no preference & herbivore & common & 31 & 14 \\
\hline & Phyllobius calcaratus & no preference & herbivore & common & 1 & 0 \\
\hline & Phyllobius maculicornis & no preference & herbivore & common & 1 & 0 \\
\hline & Polydrusus mollis & no preference & herbivore & common & 4 & 2 \\
\hline & Polydrusus pterygomalis & no preference & herbivore & common & 34 & 10 \\
\hline & Polydrusus sericeus & no preference & herbivore & common & 17 & 2 \\
\hline & Polydrusus undatus & no preference & herbivore & common & 28 & 9 \\
\hline & Rhynchaenus fagi & forest & herbivore & common & 198 & 72 \\
\hline & Sitona hispidulus & no preference & herbivore & common & 2 & 1 \\
\hline & Sitona humeralis & no preference & herbivore & common & 1 & 1 \\
\hline & Sitona lineatus & no preference & herbivore & common & 14 & 5 \\
\hline & Sitona macularius & no preference & herbivore & common & 1 & 0 \\
\hline & Sitona sp. & unknown & herbivore & unknown & 1 & 0 \\
\hline & Stereonychus fraxini & forest & herbivore & common & 5 & 1 \\
\hline & Strophosoma capitatum & no preference & herbivore & common & 1 & 0 \\
\hline & S.melanogrammum & no preference & herbivore & common & 1 & 0 \\
\hline & Tychius picirostris & no preference & herbivore & common & 0 & 1 \\
\hline Cybocephalidae & Cybocephalus politus & no preference & predator & rare & 1 & 0 \\
\hline Dasytidae & Aplocnemus nigricornis & forest & predator & common & 1 & 1 \\
\hline & Dasytes aeratus & no preference & predator & common & 9 & 3 \\
\hline & Dasytes cyaneus & forest & predator & common & 6 & 2 \\
\hline & Dasytes niger & no preference & predator & common & 1 & 0 \\
\hline & Dasytes plumbeus & no preference & predator & common & 69 & 17 \\
\hline & Trichoceble memnonia & forest & predator & rare & 1 & 0 \\
\hline Dermestidae & Attagenus pellio & no preference & omni & common & 1 & 0 \\
\hline & Ctesias serra & no preference & other & rare & 3 & 1 \\
\hline & Megatoma undata & no preference & other & rare & 15 & 4 \\
\hline Dytiscidae & Agabus sp. & unknown & predator & unknown & 1 & 0 \\
\hline & Graptodytes granularis & no preference & other & common & 1 & 0 \\
\hline & Rhantus bistriatus & no preference & predator & rare & 1 & 0 \\
\hline Elateridae & Agriotes acuminatus & no preference & herbivore & common & 30 & 2 \\
\hline & Agriotes gallicus & unknown & herbivore & rare & 1 & 1 \\
\hline & Agriotes pallidulus & no preference & herbivore & common & 7 & 0 \\
\hline & Agrypnus murina & no preference & predator & common & 2 & 0 \\
\hline & Ampedus pomorum & forest & herbivore & common & 10 & 1 \\
\hline & Athous bicolor & no preference & herbivore & rare & 2 & 1 \\
\hline & Athous haemorrhoidalis & no preference & herbivore & common & 40 & 8 \\
\hline & Athous subfuscus & no preference & herbivore & rare & 133 & 30 \\
\hline & Athous vittatus & no preference & herbivore & common & 539 & 118 \\
\hline & Calambus bipustulatus & no preference & other & rare & 8 & 1 \\
\hline & Dalopius marginatus & forest & other & common & 44 & 11 \\
\hline & Denticollis linearis & no preference & other & common & 10 & 3 \\
\hline & Denticollis rubens & forest & other & rare & 6 & 2 \\
\hline & Hemicrepidius hirtus & no preference & herbivore & common & 2 & 1 \\
\hline & Hemicrepidius niger & no preference & herbivore & common & 1 & 0 \\
\hline & Hypoganus inunctus & forest & herbivore & rare & 7 & 4 \\
\hline
\end{tabular}




\begin{tabular}{|c|c|c|c|c|c|c|}
\hline & Kibunea minutus & no preference & herbivore & common & 1 & 0 \\
\hline & Melanotus rufipes & no preference & other & common & 4 & 3 \\
\hline \multirow[t]{3}{*}{ Erotylidae } & Dacne bipustulata & no preference & fungivore & common & 55 & 15 \\
\hline & Triplax russica & no preference & fungivore & common & 2 & 0 \\
\hline & Tritoma bipustulata & unknown & fungivore & common & 3 & 1 \\
\hline \multirow[t]{4}{*}{ Eucnemidae } & Dirhagus lepidus & forest & other & rare & 2 & 0 \\
\hline & Eucnemis capucina & forest & other & rare & 4 & 0 \\
\hline & Hylis foveicollis & forest & other & rare & 2 & 0 \\
\hline & Melasis buprestoides & no preference & other & common & 1 & 0 \\
\hline \multirow[t]{3}{*}{ Helodidae } & Cyphon sp. & unknown & herbivore & unknown & 6 & 2 \\
\hline & Cyphon variabilis & unknown & herbivore & common & 4 & 1 \\
\hline & Prionocyphon serricornis & no preference & other & common & 10 & 2 \\
\hline \multirow[t]{3}{*}{ Histeridae } & Carcinops pumilio & no preference & other & common & 1 & 0 \\
\hline & Gnathoncus buyssoni & no preference & other & rare & 1 & 0 \\
\hline & Plegaderus caesus & forest & predator & common & 1 & 0 \\
\hline Hydrophilidae & Helophorus nubilus & no preference & herbivore & common & 1 & 0 \\
\hline \multirow[t]{18}{*}{ Latridiidae } & Cartodere constricta & no preference & fungivore & common & 2 & 0 \\
\hline & Cartodere nodifer & no preference & fungivore & common & 5 & 0 \\
\hline & Corticaria abietorum & forest & fungivore & rare & 10 & 3 \\
\hline & Corticaria elongata & no preference & fungivore & common & 4 & 0 \\
\hline & Corticaria polypori & forest & fungivore & rare & 4 & 0 \\
\hline & Corticaria umbilicata & no preference & fungivore & common & 2 & 2 \\
\hline & Corticarina fuscula & no preference & fungivore & common & 6 & 2 \\
\hline & Corticarina similata & no preference & fungivore & common & 99 & 37 \\
\hline & Cortinicara gibbosa & no preference & fungivore & common & 2030 & 611 \\
\hline & Enicmus atriceps & forest & fungivore & rare & 68 & 27 \\
\hline & Enicmus fungicola & forest & fungivore & common & 1 & 0 \\
\hline & Enicmus geminatus & unknown & fungivore & rare & 2 & 0 \\
\hline & Enicmus histrio & unknown & fungivore & common & 5 & 2 \\
\hline & Enicmus rugosus & forest & fungivore & common & 167 & 37 \\
\hline & Enicmus transversus & no preference & fungivore & common & 35 & 13 \\
\hline & Latridius hirtus & unknown & fungivore & rare & 63 & 23 \\
\hline & Latridius minutus & no preference & fungivore & common & 2 & 0 \\
\hline & Stephostethus alternans & forest & fungivore & rare & 4 & 1 \\
\hline \multirow[t]{3}{*}{ Leiodidae } & Agathidium nigripenne & forest & fungivore & common & 57 & 10 \\
\hline & Agathidium varians & no preference & fungivore & common & 59 & 6 \\
\hline & Anisotoma humeralis & forest & fungivore & common & 3 & 0 \\
\hline Lucanidae & Platycerus caraboides & forest & herbivore & common & 12 & 4 \\
\hline \multirow[t]{2}{*}{ Malachiidae } & Charopus flavipes & no preference & predator & common & 1 & 0 \\
\hline & Malachius bipustulatus & no preference & predator & common & 4 & 0 \\
\hline \multirow[t]{6}{*}{ Melandryidae } & Anisoxya fuscula & forest & other & rare & 10 & 7 \\
\hline & Orchesia fasciata & no preference & fungivore & rare & 1 & 0 \\
\hline & Orchesia micans & forest & fungivore & common & 1 & 1 \\
\hline & Orchesia minor & forest & fungivore & common & 19 & 4 \\
\hline & Orchesia undulata & forest & fungivore & common & 5 & 1 \\
\hline & Phloiotrya rufipes & forest & other & rare & 2 & 1 \\
\hline \multirow[t]{3}{*}{ Monotomidae } & Monotoma longicollis & no preference & unknown & common & 1 & 1 \\
\hline & Rhizophagus bipustulatus & no preference & predator & common & 85 & 23 \\
\hline & Rhizophagus parvulus & forest & predator & common & 1 & 1 \\
\hline \multirow[t]{2}{*}{ Mordellidae } & Mordellistena humeralis & unknown & other & common & 2 & 1 \\
\hline & Mordellistena neuwaldeggiana & no preference & other & common & 40 & 12 \\
\hline
\end{tabular}




\begin{tabular}{|c|c|c|c|c|c|c|}
\hline & Mordellistena variegata & unknown & other & common & 21 & 4 \\
\hline & Mordellochroa abdominalis & no preference & other & common & 28 & 2 \\
\hline \multirow[t]{4}{*}{ Mycetophagidae } & Litargus connexus & no preference & fungivore & common & 80 & 21 \\
\hline & Mycetophagus atomarius & no preference & fungivore & common & 1 & 0 \\
\hline & Mycetophagus populi & unknown & fungivore & common & 8 & 6 \\
\hline & Mycetophagus quadripustulatus & unknown & fungivore & common & 1 & 0 \\
\hline \multirow[t]{10}{*}{ Nitidulidae } & Cryptarcha strigata & forest & other & common & 13 & 1 \\
\hline & Cryptarcha undata & forest & other & common & 120 & 37 \\
\hline & Epuraea marseuli & forest & other & common & 4 & 0 \\
\hline & Epuraea melanocephala & no preference & other & common & 426 & 79 \\
\hline & Epuraea unicolor & no preference & other & common & 2 & 0 \\
\hline & Glischrochilus hortensis & no preference & other & common & 2 & 0 \\
\hline & Meligethes aeneus & no preference & herbivore & common & 9 & 6 \\
\hline & Meligethes nigrescens & no preference & herbivore & common & 1 & 0 \\
\hline & Nitidulidae sp.1 & unknown & unknown & unknown & 1 & 0 \\
\hline & Soronia grisea & no preference & other & common & 127 & 32 \\
\hline \multirow[t]{3}{*}{ Oedemeridae } & Ischnomera coerulea & forest & herbivore & rare & 3 & 2 \\
\hline & Ischnomera cyanea & forest & herbivore & common & 1 & 0 \\
\hline & Ischnomera sanguinicollis & forest & herbivore & rare & 1 & 0 \\
\hline Phalacridae & Stilbus testaceus & other & herbivore & common & 0 & 1 \\
\hline Phloiophilidae & Phloiophilus edwardsii & forest & predator & rare & 1 & 0 \\
\hline \multirow[t]{5}{*}{ Pselaphidae } & Bibloporus bicolor & forest & predator & rare & 26 & 6 \\
\hline & Bibloporus minutus & forest & predator & rare & 49 & 11 \\
\hline & Bibloporus sp. & forest & predator & rare & 2 & 0 \\
\hline & Trimium brevicorne & forest & predator & common & 8 & 2 \\
\hline & Tychus niger & unknown & predator & common & 1 & 0 \\
\hline Ptiliidae & Acrotrichis sp. & unknown & fungivore & unknown & 6 & 1 \\
\hline Ptinidae & Ptinus rufipes & forest & other & common & 1 & 1 \\
\hline \multirow[t]{2}{*}{ Pyrochroidae } & Pyrochroa coccinea & forest & other & common & 1 & 1 \\
\hline & Schizotus pectinicornis & forest & other & common & 9 & 2 \\
\hline \multirow[t]{5}{*}{ Rhynchitidae } & Byctisus betulae & no preference & herbivore & common & 0 & 1 \\
\hline & Caenorhinus pauxillus & no preference & herbivore & common & 1 & 0 \\
\hline & Deporaus betulae & no preference & herbivore & common & 3 & 0 \\
\hline & Deporaus tristis & forest & herbivore & rare & 2 & 0 \\
\hline & Lasiorhynchites olivaceus & no preference & herbivore & common & 2 & 0 \\
\hline \multirow[t]{4}{*}{ Salpingidae } & Lissodema cursor & forest & predator & rare & 12 & 2 \\
\hline & Rabocerus gabrieli & unknown & predator & rare & 14 & 5 \\
\hline & Salpingus planirostris & no preference & predator & common & 49 & 14 \\
\hline & Salpingus ruficollis & forest & predator & common & 43 & 16 \\
\hline \multirow[t]{4}{*}{ Scarabaeidae } & Aphodius fimetarius & no preference & other & common & 1 & 1 \\
\hline & Aphodius granarius & no preference & other & common & 2 & 0 \\
\hline & Aphodius prodromus & no preference & other & common & 2 & 0 \\
\hline & Gnorimus nobilis & no preference & herbivore & common & 3 & 1 \\
\hline \multirow[t]{8}{*}{ Scolytidae } & Cryphalus abietis & forest & herbivore & common & 1 & 0 \\
\hline & Cryphalus sp. & forest & herbivore & unknown & 1 & 0 \\
\hline & Crypturgus cinereus & forest & herbivore & common & 3 & 0 \\
\hline & Dryocoetes autographus & forest & herbivore & common & 5 & 1 \\
\hline & Ernoporicus cancasicus & forest & herbivore & rare & 2 & 1 \\
\hline & Ernoporicus fagi & forest & herbivore & common & 330 & 131 \\
\hline & Ernoporus tiliae & forest & herbivore & common & 2 & 1 \\
\hline & Hylastes cunicularius & forest & herbivore & common & 1 & 0 \\
\hline
\end{tabular}




\begin{tabular}{|c|c|c|c|c|c|c|}
\hline & Hylesinus crenatus & forest & herbivore & common & 4 & 1 \\
\hline & Hylesinus oleiperda & forest & herbivore & rare & 2 & 0 \\
\hline & Hylurgops palliatus & forest & herbivore & common & 1 & 0 \\
\hline & Ips cembrae & forest & herbivore & common & 1 & 0 \\
\hline & Ips typographus & forest & herbivore & common & 2 & 0 \\
\hline & Leperisinus fraxini & forest & herbivore & common & 30 & 2 \\
\hline & Pityogenes chalcographus & forest & herbivore & common & 57 & 12 \\
\hline & Polygraphus grandiclava & no preference & herbivore & common & 0 & 1 \\
\hline & Polygraphus poligraphus & forest & herbivore & common & 34 & 26 \\
\hline & Scolytus carpini & forest & herbivore & common & 30 & 7 \\
\hline & Scolytus intricatus & forest & herbivore & common & 7 & 8 \\
\hline & Taphrorychus bicolor & forest & herbivore & common & 201 & 66 \\
\hline & Xyleborus peregrinus & forest & fungivore & rare & 270 & 78 \\
\hline & Xyleborus saxeseni & no preference & fungivore & common & 25 & 6 \\
\hline & Xyleborus sp. & unknown & fungivore & unknown & 7 & 0 \\
\hline Scraptiidae & Anaspis flava & no preference & other & common & 14 & 3 \\
\hline & Anaspis frontalis & no preference & other & common & 16 & 1 \\
\hline & Anaspis melanostoma & no preference & other & rare & 10 & 4 \\
\hline & Anaspis rufilabris & no preference & other & common & 64 & 17 \\
\hline & Anaspis thoracica & no preference & other & common & 63 & 13 \\
\hline Scydmaenidae & Neuraphes elongatulus & no preference & predator & common & 3 & 1 \\
\hline & Stenichnus bicolor & forest & predator & rare & 1 & 0 \\
\hline & Stenichnus scutellaris & forest & predator & common & 1 & 1 \\
\hline Silvanidae & Silvanus unidentatus & forest & predator & common & 1 & 0 \\
\hline & Uleiota planata & no preference & predator & common & 3 & 1 \\
\hline Sphindidae & Arpidiphorus orbiculatus & no preference & fungivore & common & 1 & 0 \\
\hline Staphylinidae & Acrotona sp. & unknown & predator & unknown & 4 & 1 \\
\hline & Aleochara bipustulata & no preference & predator & common & 4 & 1 \\
\hline & Aleochara sparsa & no preference & predator & common & 28 & 5 \\
\hline & Aleochara laevigata & no preference & predator & rare & 1 & 0 \\
\hline & Aleochara sanguinea & no preference & predator & common & 1 & 0 \\
\hline & Aleochara sp. & unknown & predator & unknown & 3 & 1 \\
\hline & Aloconota gregaria & no preference & predator & common & 10 & 2 \\
\hline & Amarochara bonnairei & no preference & predator & rare & 3 & 0 \\
\hline & Amischa analis & no preference & predator & common & 3 & 0 \\
\hline & Amischa decipiens & no preference & predator & common & 3 & 0 \\
\hline & Amischa sp. & no preference & predator & unknown & 53 & 13 \\
\hline & Anomognathus cuspidatus & forest & predator & common & 3 & 1 \\
\hline & Anotylus inustus & no preference & other & common & 5 & 1 \\
\hline & Anotylus rugosus & no preference & other & common & 1 & 0 \\
\hline & Anotylus sculpturatus & no preference & other & common & 4 & 3 \\
\hline & Anotylus tetracarinatus & no preference & other & common & 19 & 6 \\
\hline & Anthobium atrocephalum & no preference & other & common & 3 & 0 \\
\hline & Anthophagus angusticollis & unknown & predator & common & 5 & 2 \\
\hline & Atheta amplicollis & unknown & predator & common & 2 & 2 \\
\hline & Atheta fungi & no preference & predator & common & 381 & 116 \\
\hline & Atheta inquinula & no preference & predator & common & 4 & 2 \\
\hline & Atheta nigra & no preference & predator & common & 0 & 1 \\
\hline & Atheta palustris & unknown & predator & common & 1 & 1 \\
\hline & Atheta sp. & unknown & predator & unknown & 74 & 28 \\
\hline & Atheta sp.1 & unknown & predator & unknown & 1 & 1 \\
\hline
\end{tabular}




\begin{tabular}{|c|c|c|c|c|}
\hline Atheta sp.2 & unknown & predator & unknown & 1 \\
\hline Atheta triangulum & no preference & predator & common & 1 \\
\hline Bolitobius castaneus & no preference & predator & rare & 1 \\
\hline Bolitobius sp. & no preference & predator & rare & 1 \\
\hline Bythinus burrelli & no preference & predator & common & 2 \\
\hline Callicerus obscurus & no preference & predator & common & 1 \\
\hline Carpelimus corticinus & unknown & other & common & 1 \\
\hline Cypha longicornis & no preference & predator & common & 3 \\
\hline Cyphea curtula & forest & predator & rare & 19 \\
\hline Euplectus karsteni & no preference & predator & common & 4 \\
\hline Euplectus punctatus & forest & predator & rare & 3 \\
\hline Euryusa optabilis & forest & predator & common & 2 \\
\hline Eusphalerum luteum & no preference & herbivore & common & 65 \\
\hline Eusphalerum minutum & unknown & herbivore & common & 6 \\
\hline E.pseudancupariae & unknown & herbivore & rare & 40 \\
\hline Eusphalerum sp. & unknown & herbivore & unknown & 1 \\
\hline Geostiba circellaris & no preference & predator & common & 3 \\
\hline Haploglossa picipennis & forest & predator & rare & 1 \\
\hline Haploglossa villosula & no preference & predator & common & 50 \\
\hline Heterothops niger & no preference & predator & common & 1 \\
\hline Holobus flavicornis & no preference & predator & common & 26 \\
\hline Hypnogyra glabra & no preference & predator & common & 1 \\
\hline Ischnoglossa sp. & unknown & predator & rare & 4 \\
\hline Ischnosoma splendidum & no preference & predator & common & 4 \\
\hline Lathrobium elongatum & no preference & predator & common & 3 \\
\hline Leptacinus $s p$. & unknown & predator & unknown & 1 \\
\hline Leptusa fumida & forest & predator & common & 10 \\
\hline Leptusa ruficollis & forest & predator & common & 7 \\
\hline Lesteva longoelytrata & unknown & predator & common & 4 \\
\hline Liogluta nitidula & no preference & predator & common & 1 \\
\hline Liogluta sp. & unknown & predator & unknown & 8 \\
\hline Meotica sp. & unknown & predator & rare & 1 \\
\hline Metopsia clypeata & no preference & fungivore & rare & 6 \\
\hline Mycetoporus solidicornis & no preference & predator & rare & 1 \\
\hline Ocalea $\mathrm{sp}$. & unknown & predator & unknown & 1 \\
\hline Oligota pusilima & no preference & predator & unknown & 0 \\
\hline Oligota sp. & unknown & predator & unknown & 10 \\
\hline Omalium caesum & no preference & other & common & 3 \\
\hline Omalium rivulare & no preference & other & common & 1 \\
\hline Oxypoda brevicornis & no preference & predator & common & 1 \\
\hline Oxypoda sp. & unknown & predator & unknown & 2 \\
\hline Philonthus carbonarius & no preference & predator & common & 16 \\
\hline Philonthus cognatus & no preference & predator & common & 23 \\
\hline Philonthus coracinus & unknown & unknown & unknown & 4 \\
\hline Philonthus decorus & forest & predator & common & 1 \\
\hline Philonthus rotundicollis & no preference & predator & common & 8 \\
\hline Philonthus sp. & unknown & predator & unknown & 1 \\
\hline Phloeopora corticalis & no preference & predator & common & 496 \\
\hline Phloeopora testacea & forest & predator & common & 8 \\
\hline Phyllodrepa floralis & no preference & predator & common & 1 \\
\hline Phyllodrepa ioptera & no preference & predator & common & 15 \\
\hline
\end{tabular}




\begin{tabular}{|c|c|c|c|c|c|c|}
\hline & Placusa atrata & forest & predator & common & 1 & 1 \\
\hline & Placusa depressa & forest & predator & common & 2 & 0 \\
\hline & Plataraea brunnea & no preference & predator & common & 7 & 2 \\
\hline & Platystethus nitens & no preference & other & common & 7 & 1 \\
\hline & Quedius brevicornis & no preference & predator & rare & 3 & 0 \\
\hline & Quedius fuliginosus & no preference & predator & common & 1 & 1 \\
\hline & Quedius invrae & no preference & predator & common & 1 & 0 \\
\hline & Quedius maurus & forest & predator & rare & 24 & 7 \\
\hline & Quedius ochripennis & no preference & predator & common & 2 & 0 \\
\hline & Rugilus erichsoni & no preference & predator & rare & 0 & 1 \\
\hline & Rugilus rufipes & no preference & predator & common & 1 & 0 \\
\hline & Scaphisoma agaricinum & other & fungivore & common & 2 & 0 \\
\hline & Silusa rubiginosa & forest & predator & common & 1 & 0 \\
\hline & Stenus clavicornis & no preference & predator & common & 1 & 0 \\
\hline & Stenus similis & no preference & predator & common & 1 & 1 \\
\hline & Stichoglossa semirufa & no preference & predator & rare & 61 & 12 \\
\hline & Tachinus sp. & unknown & predator & unknown & 2 & 0 \\
\hline & Tachyporus atriceps & no preference & predator & common & 2 & 1 \\
\hline & Tachyporus hypnorum & no preference & predator & common & 46 & 12 \\
\hline & Tachyporus nitidulus & no preference & predator & common & 21 & 6 \\
\hline & Tachyporus obtusus & unknown & predator & common & 3 & 0 \\
\hline & Tachyporus solutus & no preference & predator & common & 8 & 4 \\
\hline & Xantholinus linearis & no preference & predator & common & 19 & 7 \\
\hline Tenebrionidae & Diaperis boleti & forest & fungivore & common & 13 & 3 \\
\hline & Latheticus oryzae & unknown & fungivore & rare & 1 & 0 \\
\hline & Palorus depressus & no preference & other & rare & 1 & 0 \\
\hline & Platydema violaceum & unknown & other & rare & 1 & 0 \\
\hline Tetratomidae & Tetratoma ancora & unknown & fungivore & common & 28 & 8 \\
\hline Throscidae & Aulonothroscus brevicollis & forest & herbivore & common & 4 & 0 \\
\hline & Trixagus dermestoides & no preference & unknown & common & 1 & 0 \\
\hline & Trixagus sp. & unknown & unknown & unknown & 3 & 0 \\
\hline Trogossitidae & Nemosoma elongatum & no preference & predator & common & 10 & 3 \\
\hline
\end{tabular}




\section{Appendix 2.3}

Spearman rank correlations of untransformed response (beetle richness) and explanatory variables.

\begin{tabular}{|c|c|c|c|c|c|c|c|c|c|c|}
\hline & $\begin{array}{l}\text { Beetle } \\
\text { Richness }\end{array}$ & $\begin{array}{l}\text { Beetle } \\
\text { Abundance }\end{array}$ & $\begin{array}{l}\text { Tree Diversity } \\
\text { (Shannon) }\end{array}$ & $\begin{array}{l}\text { Stand } \\
\text { Age }\end{array}$ & Stem Density & $\begin{array}{l}\text { Tree } \\
\text { Height }\end{array}$ & $\begin{array}{l}\text { Crown } \\
\text { Area }\end{array}$ & $\begin{array}{l}\text { Plant } \\
\text { Index }\end{array}$ & Area & $\begin{array}{l}\text { Deadwood } \\
\mathrm{m}^{3} / \mathrm{m}^{2}\end{array}$ \\
\hline Beetle Richness & 1 & & & & & & & & & \\
\hline Beetle Abundance & 0.85 & 1 & & & & & & & & \\
\hline Tree Diversity (Shannon) & 0.94 & 0.7 & 1 & & & & & & & \\
\hline Stand Age & -0.7 & -0.35 & -0.81 & 1 & & & & & & \\
\hline Stem Density & 0.18 & -0.18 & 0.34 & -0.72 & 1 & & & & & \\
\hline Tree Height & -0.68 & -0.36 & -0.78 & 0.95 & -0.63 & 1 & & & & \\
\hline Crown Area & -0.32 & 0.01 & -0.49 & 0.84 & -0.92 & 0.69 & 1 & & & \\
\hline Plant Area Index & 0.57 & 0.3 & 0.65 & -0.75 & 0.46 & -0.69 & -0.6 & 1 & & \\
\hline Dead Wood Volume & -0.3 & -0.05 & -0.36 & 0.66 & -0.54 & 0.77 & 0.51 & -0.21 & & 1 \\
\hline
\end{tabular}




\section{Appendix 2.4}

Relative contributions of $\alpha$ - and $\beta$-diversity to beetle species richness of communities sampled on various tree species in 12 forest plots across a tree diversity gradient. Values for beech alone are set in parentheses. Stands were ranked based on increasing Shannon Indices (tree diversity), rank 3 was assigned twice due to concording Shannon indices.

\begin{tabular}{llll}
\hline Forest Stand $\% \alpha$ & $\% \beta_{\text {time }}$ & $\% \beta_{\text {space }}$ \\
\hline 1 & $9.4(17.7)$ & $29.2(55.6)$ & $61.4(26.7)$ \\
2 & $8.9(15.1)$ & $28.2(49.9)$ & $62.9(35)$ \\
$3 \mathrm{a}$ & $10(15.9)$ & $29.4(51.7)$ & $60.6(32.4)$ \\
$3 \mathrm{~b}$ & $9.7(17.1)$ & $28.9(49.5)$ & $61.4(33.3)$ \\
4 & $8.3(15.6)$ & $26.6(50.8)$ & $65.1(33.6)$ \\
5 & $9.6(14.1)$ & $26.3(52.4)$ & $64.1(33.6)$ \\
6 & $8.9(14.2)$ & $29.2(49.8)$ & $61.8(36)$ \\
7 & $7.8(14)$ & $26(52.6)$ & $66.2(33.3)$ \\
8 & $10(30.3)$ & $24.3(31.6)$ & $65.7(38.1)$ \\
9 & $8.5(15.3)$ & $27.2(49.5)$ & $64.4(35.2)$ \\
10 & $10.2(17)$ & $29.9(50.6)$ & $59.9(32.4)$ \\
11 & $8.6(13.9)$ & $27.8(51.3)$ & $63.6(34.8)$ \\
\hline
\end{tabular}




\section{Appendix 2.5}

Relative contributions of species abundance to functional guilds for canopy beetles captured on various tree species across a tree diversity gradient in the Hainich National Park. Values for beech alone are set in parentheses. Stands were ranked based on increasing Shannon Indices (tree diversity), rank 3 was assigned twice due to concording Shannon indices.

\begin{tabular}{|c|c|c|c|c|c|c|c|}
\hline Forest & Habitat & Forest & $\%$ Common & Rare & $\%$ & $\%$ & $\%$ \\
\hline Stand & Generalists & Species & Species & Species & Predators & Herbivores & Fungivores \\
\hline 1 & 61.6 & $4.1)$ & 00.0 & $3.8)$ & $37.4(39.7)$ & $5.3(24.1)$ & 5) \\
\hline 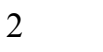 & $58.6(58.6)$ & $26.1(28.6)$ & $77.5(82.9)$ & $15.3(12.9)$ & $34.2(34.3)$ & $29.7(27.1)$ & $18(21.4)$ \\
\hline $3 \mathrm{a}$ & $52.5(55.9)$ & $33.1(29.4)$ & $76.3(77.9)$ & $18.6(16.2)$ & $33.1(30.9)$ & $22(22.1)$ & $17.8(22.1)$ \\
\hline $3 b$ & 5 & 3 & 7 & (- & $3.9(30.3)$ & 1 & 2) \\
\hline 4 & $58.4(65.6)$ & $30.4(20.3)$ & 78.4 (79.7) & $17.6(15.6)$ & $40(40.6)$ & $23.2(31.3)$ & $13.6(10.9)$ \\
\hline 5 & $56.6(59.7)$ & $30.3(25.4)$ & $83.4(85.1)$ & $13.1(13.4)$ & $35.9(38.8)$ & 31.7 (26.9) & $13.8(13.4)$ \\
\hline 6 & $59.4(56.1)$ & $26.1(28)$ & $75.4(76.8)$ & $19.6(18.3)$ & $34.8(28)$ & $26.8(34.1)$ & $13.8(14.6)$ \\
\hline 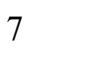 & $55.2(61.9)$ & $29.1(25.4)$ & $76.1(84.1)$ & $14.9(9.5)$ & $33.6(31.7)$ & $27.6(30.2)$ & $16.4(17.5)$ \\
\hline 8 & .7) & $28.5(2$ & $76.4(80.6)$ & $17(13.4)$ & $35.8(4$ & 29.7 & $13.9(17.9)$ \\
\hline 9 & $58.3(63)$ & $23.3(25.9)$ & $75(80.2)$ & $17.2(14.8)$ & $33.9(37)$ & $28.9(24.7)$ & $15.6(13.6)$ \\
\hline 10 & $59.6(58)$ & $28.7(31.8)$ & $78.4(77.3)$ & $16.4(17)$ & $36.3(36.4)$ & $26.9(26.1)$ & $15.8(15.9)$ \\
\hline 11 & $58.6(64.6)$ & $27.1(25.3)$ & $76.8(86.1)$ & $16.6(10.1)$ & $35.9(35.4)$ & $27.6(30.4)$ & $14.9(12.7)$ \\
\hline
\end{tabular}




\section{CHAPTER}

\section{3}

\section{Room for more is in the mix: tree diversity drives spatiotemporal $\beta$-diversity of true bugs (Heteroptera)}

S. Sobek, M. Goßner, C. Scherber, I. Steffan-Dewenter, T. Tscharntke (submitted) 


\begin{abstract}
The forest canopy is a major hot-spot of insect diversity, but almost nothing is known for functionally important and abundant taxa such as true bugs (Heteroptera). Spatiotemporal patterns of true bug diversity in forests of different tree diversity have not yet been disentangled, although plant diversity has been shown to strongly impact the diversity and distribution of many insect communities. Here we compare species richness of canopy true bugs across a tree diversity gradient ranging from simple beech to mixed forest stands. We analyse changes in community composition by additive partitioning of species diversity, for communities on various tree species as well as for communities dwelling on beech alone. Total species richness ( $\gamma$-diversity), $\alpha$-diversity, and abundance of true bugs increased across the tree diversity gradient, but changes were mediated by increased true bug abundance in the highly diverse forest stands. The same pattern was found for $\gamma$-diversity in most functional guilds (e. g. forest specialists, herbivores, predators). Temporal and even more, spatial turnover ( $\beta$-diversity) among trees was closely related to tree diversity and accounted for $\sim 90$ $\%$ of total $\gamma$-diversity. Patterns for beech were similar, but species turnover could not be related to the tree diversity gradient, and on beech alone, monthly turnover was generally higher compared to turnover among trees. Our findings support the hypothesis that with increasing tree diversity and thereby increasing habitat heterogeneity, enhanced resource availability supports a greater number of individuals and species of true bugs in the more diverse forest stands. We conclude that simple beech stands lack capacity for conserving insect species richness of deciduous forest habitats, and show that tree species identity and the dissimilarity of true bug communities from tree to tree matters when analysing community patterns. To understand diversity and distribution of insect communities in forest habitats, future research needs a better integration of previously neglected groups and of the extensive spatiotemporal community changes involved.
\end{abstract}

Key words: Beta diversity, biodiversity conservation, canopy arthropods, Fagus sylvatica L., functional groups, habitat heterogeneity 


\section{Introduction}

It has long been known that especially tree crowns in tropical forests house the key proportion of global insect diversity (Stork 1988). Only more recently, the insect fauna of the forest canopy in temperate and boreal forests has gained major attention, and species diversity and distribution in these habitats has been assessed in a variety of studies (e.g. Ozanne 1996, Thunes et al. 2003, Southwood et al. 2005, Ulyshen \& Hanula 2007). The majority of published studies focus on forest stands differing in management intensity, stand age, deadwood amount or other parameters. Furthermore, they include only a small selection of tree species, usually comparing deciduous with conifer trees. Up to now, information on exclusively deciduous forest stands that a priori differ in tree diversity is lacking, and standwise canopy insect richness has rarely been linked to overall canopy tree diversity of the sampled stands. Especially spatiotemporal patterns of insect diversity in temperate deciduous forest monocultures have not yet been elucidated, and it remains unexplored how they compare to patterns in mixed stands within the same forest neighbourhood.

Most examinations of arthropod species richness in forest ecosystems have primarily been limited to a single spatial or temporal scale, which can lead to misleading results if differences occur elsewhere but not at the observed scale. Choosing the right scale is of particular importance to pinpoint diversity patterns, and some authors have recently reported noteworthy changes across multiple spatiotemporal scales by using trap-nesting bees and wasps (Tylianakis et al. 2005), or butterflies and beetles (Hirao et al. 2007) as model systems. As listed in Tylianakis et al. (2006), diversity of arthropods is largely scale-dependent and driven by various factors which change if larger spatial scales (e.g. regions) are compared to smaller spatial scales (e. g. single trees). Hence, faint differences in species richness across spatiotemporal scales might have previously remained undiscovered, especially for taxonomic groups that have not yet gained major attention among forest researchers, like true bugs (Heteroptera).

Whilst well-described taxa such as beetles or butterflies have a long tradition in being used as study systems in forest ecosystems, true bugs have up to now widely been neglected. The latter is surprising, because as a result of their high degree in host plant specialisation and based on their feeding habits as sucking insects, true bugs might respond differently to environmental gradients compared to the groups mentioned above. Moreover, this taxonomical bias apparently is limited to forest ecosystems. In other habitats, such as aquatic (Polhemus \& Polhemus 2008) or agricultural ecosystems (Fauvel 1999), true bugs have 
intensively been studied not only due to their important functional roles as predators and herbivores, but also because of their use as indicator group for overall arthropod species richness within one habitat (Duelli \& Obrist 1998).

One approach to assess diversity across different scales in space and time is the additive partitioning of diversity (Lande 1996, Veech et al. 2002, Crist et al. 2003), which is a ubiquitously applied method to determine species turnover ( $\beta$-diversity) of insect communities in various habitat types. By additive partitioning, diversity is split up in several components: $\gamma$-diversity (total diversity across several sampled units), $\alpha$-diversity (average diversity within a single sampled unit), and $\beta$-diversity (difference between $\gamma$ and $\alpha$ ). Hence, higher $\beta$-diversity indicates increased differences in community compostion, or in other words, a higher turnover of species in space or time (Clough et al. 2007).

Here we apply additive partitioning to canopy true bug diversity at multiple spatiotemporal scales across a tree diversity gradient ranging from simple beech to mixed deciduous forest stands. To our knowledge this is the first attempt to determine at which spatial or temporal scale the highest diversity occurs for this taxon, specifically in a forest habitat. Our analyses include true bug communities on various tree species in the mix as well as communities on single beech trees (Fagus sylvatica L.) across the gradient. Recently acknowledged as being the most important and successful plant species in Europe (Leuschner et al. 2006), it is of specific concern what role beech plays in maintaining arthropod diversity in temperate forest ecosystems.

We expect communities of true bugs in simple beech stands to be more similar to each other than to communities in species rich stands, although diversity of communities on single beech trees within an otherwise mixed forest neighbourhood might also increase due to species spillover. To test our hypothesis, we apply an analysis of the distribution and species richness of functional groups and feeding guilds across the tree diversity gradient as well as additive partitioning of true bug diversity.

In particular, we test the following predictions with regard to effects of tree diversity on species richness and community composition of true bugs: (i) Habitat complexity has often been found to positively correlate with arthropod species richness (Humphrey et al. 1999, Hansen 2000, Hamer et al. 2003, Lassau \& Hochuli 2008), hence we expect species richness of true bugs to be higher in more diverse forest stands as a result of increased structural complexity. (ii) Many true bug species show a high degree of host plant affiliation, either as food or habitat resource (Wachmann et al. 2004, Goßner 2008). In line with increased abundance of tree and herb layer plant species in the most diverse forest stands, we presume 
higher true bug species turnover ( $\beta$-diversity) in these habitats, due to increased abundance of specialists and forest-dwelling species restricted to particular tree species as habitat or food resource. (iii) For the same reason, we assume that seasonal turnover $\left(\beta_{\text {time }}\right)$ contributes less to overall diversity compared to spatial turnover $\left(\beta_{\text {space }}\right)$ between trees.

\section{Materials and Methods}

\section{Study site description}

Sampling was carried out in the Hainich National Park, which is located in Central Germany (Thuringia) north of the city of Eisenach. With a total area of 16,000 ha (7,600 ha designated as national park), the area is depicted as Germany's largest remaining semi-natural deciduous forest (Nationalpark Hainich; http://www.nationalpark-hainich.de). Prior to the German Reunion, the area was used as a military training site and has in large parts remained umanaged for at least 60 years, resulting in a late-succesional state of deciduous temperate forest which has been declared national park in 1997. The region has a mild temperate climate, with an average temperature of $7.5^{\circ} \mathrm{C}$ and a mean precipitation of $590 \mathrm{~mm}$ (19732004, Deutscher Wetterdienst). The average annual temperature in 2005 was $9{ }^{\circ} \mathrm{C}$, annual precipitation in 2005 was $601 \mathrm{~mm}$ (Meteomedia 2005).

A major characteristic of the Hainich National Park is the lack of conifers in favour of a wide variety of deciduous tree species. Besides stands dominated by European beech (Fagus sylvatica L.), mixed forests with a high proportion of lime (Tilia cordata L., Tilia platyphyllos Scop.) and ash (Fraxinus excelsior L.) can also be found, and further deciduous tree species like hornbeam (Carpinus betulus L.), maple (Acer pseudoplatanus L. , Acer platanoides L., Acer campestre L.), oak (Quercus robur L.), elm (Ulmus glabra Huds.), cherry (Prunus avium L.) and service tree (Sorbus torminalis L.) add variety to the mix.

For the present study, 12 forest stands of different tree diversity were chosen for examination, thereby establishing a tree diversity gradient ranging from simple beech to mixed forest stands with up to 11 deciduous tree species. All stands exhibited equal site characteristics in terms of soil (stagnic luvisol on loess-limestone as parent material), flat elevation, absence of canopy gaps and timespan since last management event (40 years).

Tree diversity of each stand was determined by recording tree species and individual abundance in a $50 \times 50$ sampling plot. To account for species richness and eveness (Magurran 2004), tree diversity in all subsequent analyses will be expressed as Shannon index based on stem counts of individuals with a diameter breast height $(\mathrm{dbh})>7 \mathrm{~cm}$ (Appendix 3.1). 


\section{Sampling design}

True bugs were sampled with flight interception traps mounted in the forest canopy. Flight interception traps are commonly used to capture flying insects in forest habitats (e.g. Goßner et al. 2007, Müller et al. 2008). The traps consisted of two translucent polycarbonate panes $(60 \times 40 \mathrm{~cm})$, which were arranged cross-shaped to maximise the probability that insects flying in from any direction hit one of the panes in flight. The insects then either drop down into a funnel leading to lower collecting jar, or fly up the window pane into a funnel attached to an upper collecting jar, both filled with ethylene-glycol (1:1 diluted with water) as a killing and preserving liquid.

Across all forest stands, 72 traps (six traps per stand) were suspended in the centre of individual tree crowns using a crossbow and following a sampling design based on the proportion of beech. In beech dominated stands (83-100\% beech) with a maximum of 3 other tree species, only beech (Fagus sylvatica) was sampled, in stands with at least $48 \%$ beech and up to six other tree species, the three most dominant species were sampled (beech, lime (Tilia sp.), ash (Fraxinus excelsior), and in stands with the relative abundance of beech declining below $42 \%$ and up to ten other tree species, six tree species were sampled (beech, lime, ash, sycamore maple (Acer pseudoplatanus), hornbeam (Carpinus betulus) and either oak (Quercus robur) or service tree (Sorbus torminalis)). Sampled trees were randomly chosen on the $50 \times 50 \mathrm{~m}$ plots or in the directly adjacent neighbourhood. To include a comparison of single beech trees across the gradient, four supplementary traps were mounted in beech trees of the four most diverse forest stands. Analyses for beech alone were then derived from two randomly drawn/sampled trees per stand. All traps were cleared in intervals of four weeks over a period of six months (May to October 2005).

\section{Sample processing}

All true bugs were separated from bycatches, debris and plant material and preserved in $70 \%$ ethyl alcohol. All specimens were identified to species level, except for some damaged and juvenile individuals that were assigned to higher taxonomic levels. Alcohol preserved voucher specimens were stored in the in-house collection (Agroecology, Georg-August-University Göttingen). Every species was supplied with additional information on habitat specialisation, stratum preference, adult feeding guild (predators: zoophagous species and zoophytophagous species with predominantly zoophagous diet during adult stage, herbivores: strictly phytophagous species and zoophytophagous species with predominantly phytophagous diet during adult stage), host plant specialisation (food or habitat resource) and prey specialisation 
as outlined in Rabitsch et al. (in press) (Appendix 3.2). Feeding guilds were classified based on adult diet because flight-interception traps predominantly sample imagines.

\section{Statistical analyses}

Data analyses were conducted separately for six sampled trees per stand (beech or a mix of species), or for two sampled beech trees per stand. Observed species richness ( $\gamma$-diversity) of true bugs was expressed as accumulated number of species per stand (pooled over space and time). We applied a non-parametric species richness estimator (first order Jackknife) to calculate sampling success, which was similar across the gradient (64-83\% of estimated species, Appendix 3.1).

Additive partitioning of $\gamma$-diversity was conducted for each forest stand following Lande 1996), wherein $\gamma$ (overall regional diversity) $=\alpha$ (mean species richness within sample) $+\beta$ (species turnover). Here, we partitioned $\gamma_{\text {Stand }}$ in $\alpha+\beta_{\text {time }}+\beta_{\text {space, }}$ with $\alpha$ defined as mean species richness per tree per month, seasonal turnover $\beta_{\text {time }}$ as mean $\beta_{\text {timeTree }}(=$ observed number of species per tree minus $\alpha$ ), and spatial turnover $\beta_{\text {space }}$ specified as observed species richness per plot minus mean number of species per tree (pooled over the sampling season).

Relationships of the response variables ( $\gamma$-diversity, rarefied $\gamma$-diversity, $\alpha, \beta_{\text {time }}, \beta_{\text {space, }}$, species richness in functional guilds) with tree diversity were tested with multipe regression models. To reach assumtion of normality and homogeneity of variance, count data were $\log _{10}$ transformed and proportional data arcsine-squareroot transformed prior to calculation of the model. Models were calculated using type I sum of squares with (1) true bug abundance and (2) tree diversity (Shannon index) as explanatory variables. The study was a priori designed to test effects of tree diversity. Tree diversity controlled several other biotic and abiotic plot characteristics (Appendix 3.3) which hence were not included in the statistical models for reasons of multicollinearity. True bug abundance data was included for correction of sample size. In the analyses of various tree species per forest stand, true bug abundance was strongly positively affected by tree diversity (Pearson's $\rho=0.91, \mathrm{p}<0.001$ ), hence we tested different sequences of variables entered to the model. The same accounted for beech alone (Pearson's $\rho$ $=0.84, \mathrm{p}<0.001)$. For each response variable, models were fitted separately, and analysis of covariance (ANCOVA) was used to test for differences in regression slopes of $\alpha, \beta_{\text {time }}$ and

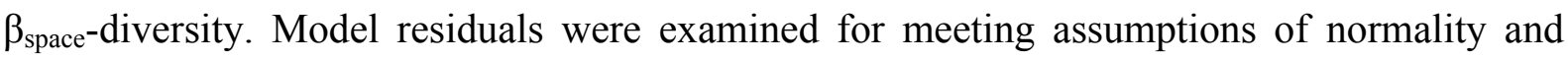
homogeneity of variance. Computation of first order Jackknife estimates was compiled with EstimateS 8.0 (Colwell 2006). The remaining statistical analyses were carried out with $\mathrm{R}$, Version 2.6.2 (R Development Core Team 2007; http://www.R-project.org). 


\section{Results}

\section{True bug community}

In total, 2,431 individuals and 76 species were trapped within a period of six months in 2005 (Appendix 3.2). The most common species was Deraeocoris lutescens Schilling (708 individuals, $29.1 \%$ ), followed by Psallus varians Herrich-Schaeffer (433 individuals, $17.8 \%$ ) and Harpocera thoracica Fallén (203 individuals, $8.4 \%$ ). The remaining 73 species (96.1\%) each contributed less than $5 \%$ to the total of sampled individuals, 21 species $(27.6 \%)$ were singletons. On beech trees, 522 individuals and 43 species and were captured, 18 (41.9\%) species thereof singletons (Appendix 3.2). Again, the predator Deraeocoris lutescencs was most abundant (171 individuals, $32.8 \%$ ), but only two other species, Psallus varians (123 individuals, $23.6 \%$ ) and Pentatoma rufipes L., reached abundance levels above $5 \%$ (28 individuals, $5.4 \%$ ). The vast majority of species on beech remained below $1 \%$ in abundance (31 species, $72.1 \%$ ).

\section{Species richness and additive partitioning of diversity}

Observed species richness ( $\gamma$-diversity) was not affected by tree diversity after controlling for increased individual abundance across the gradient (Abundance: $F_{1,9}=51.88, \mathrm{p}<0.001$, Shannon index: $F_{1,9}=0.51, \mathrm{p}=0.495$, Table 3.1). Nevertheless, the number of observed species tripled with increased tree diversity (Fig. 3.1), and the effect was significant when tree diversity was introduced to the multiple regression model first (Shannon index: $F_{1,9}=47.11, \mathrm{p}$ $<0.001$, Abundance: $F_{1,9}=5.28, \mathrm{p}=0.047$, Table 3.1). Considering beech only, the number of observed true bug species also tripled across the gradient (Fig. 3.1), but the response to tree diversity was again primarily controlled by increased individual abundance (Shannon index, $F_{1,9}=8.36, \mathrm{p}=0.018$, Abundance: $F_{1,9}=47.11, \mathrm{p}<0.001$, Table 3.1).

The same effect explained increased $\alpha$-diversity across the sampled forest stands (Shannon index: $F_{1,9}=280.23, \mathrm{p}<0.001$, Abundance: $F_{1,9}=36.57, \mathrm{p}<0.001$, Table 3.1). Both $\beta_{\text {time }}$ (Shannon index: $F_{1,9}=45.79, \mathrm{p}<0.001$, Table 3.1) and $\beta_{\text {space-diversity (Shannon index: } F_{1,9}=}$ 18.05, $\mathrm{p}=0.001$, Table 3.1) also increased across the tree diversity gradient, but spatial

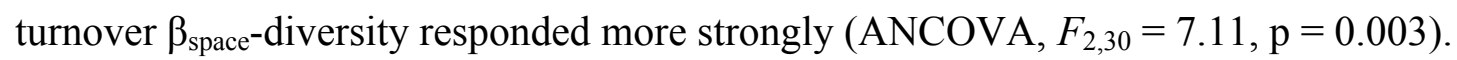


Table 3.1. Multiple regression analyses of species richness parameters for canopy true bugs across a tree diversity gradient in the Hainich National Park. Values for beech alone are set in parentheses. ${ }^{\dagger}$ Temporal and spatial species turnover was not linked to turnover in true bug abundance, hence this variable was omitted from the model

\begin{tabular}{|c|c|c|c|c|c|c|c|}
\hline \multirow[b]{2}{*}{ Response Variable } & \multirow[b]{2}{*}{ Effect } & \multicolumn{3}{|c|}{$\begin{array}{l}\text { Model A: } \\
\text { Abundance }+ \text { Shannon Index }\end{array}$} & \multicolumn{3}{|c|}{$\begin{array}{l}\text { Model B: } \\
\text { Shannon Index }+ \text { Abundance }\end{array}$} \\
\hline & & Multiple r2 & $F$ & $\mathrm{p}$ & Multiple r2 & $F$ & $\mathrm{p}$ \\
\hline \multirow[t]{2}{*}{$\gamma$-Diversity } & Abundance & & $51.88(14.06)$ & $<0.001(0.005)$ & & $5.28(5.72)$ & $0.047(0.04)$ \\
\hline & Shannon Index & $0.85(0.61)$ & $0.51(0.01)$ & $0.495(0.906)$ & $0.85(0.61)$ & $47.11(8.36)$ & $<0.001(0.018)$ \\
\hline \multirow[t]{2}{*}{$\alpha$-Diversity } & Abundance & & 313.78 (49.79) & $<0.001(<0.001)$ & & $36.57(23.01)$ & $<0.001(<0.001)$ \\
\hline & Shannon Index & $0.97(0.85)$ & $3.02(0.02)$ & $0.116(0.901)$ & $0.97(0.85)$ & $280.23(26.79)$ & $<0.001(<0.001)$ \\
\hline \multirow[t]{2}{*}{$\beta_{\text {time}}$-Diversity } & Abundance $^{\dagger}$ & & & & & & \\
\hline & Shannon Index & $0.82(0.31)$ & $45.79(4.5)$ & $<\mathbf{0 . 0 0 1}(0.06)$ & & & \\
\hline \multirow[t]{2}{*}{$\beta_{\text {space }}$-Diversity } & Abundance $^{\dagger}$ & & & & & & \\
\hline & Shannon Index & $0.64(0.19)$ & $18.05(2.4)$ & $\mathbf{0 . 0 0 1}(0.152)$ & & & \\
\hline
\end{tabular}




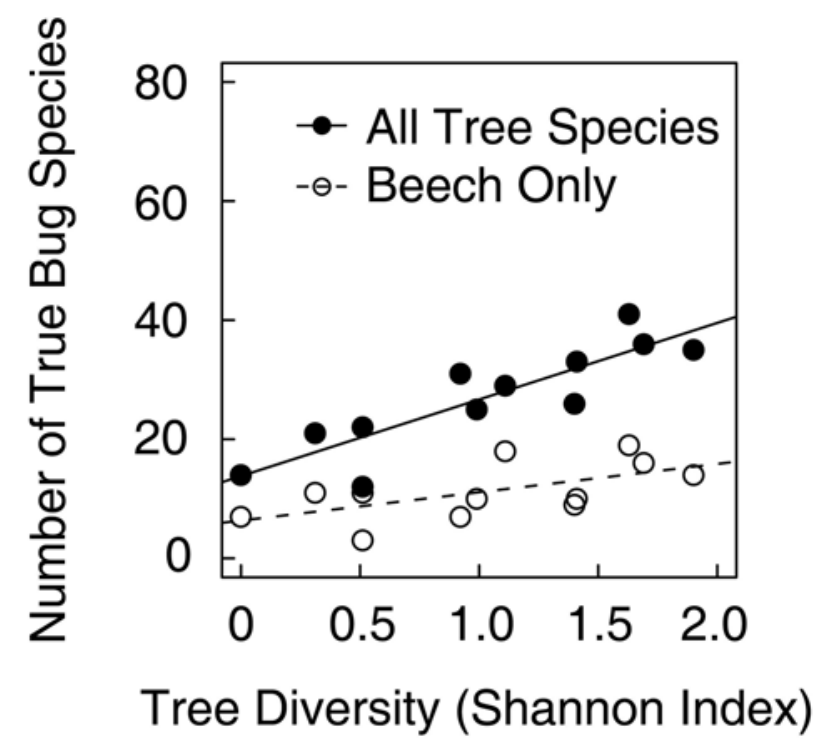

Fig. 3.1. Observed species richness ( $\gamma$-diversity) of true bugs across a tree diversity gradient in a Central European deciduous forest.

Including all tree species in the analysis, spatial turnover contributed more to $\gamma$-diversity than temporal turnover (Fig. 3.2a), but the pattern reversed if beech alone was considered (Fig $3.2 b)$. For beech trees, temporal and spatial turnover of true bug species did not respond to tree diversity, even if tree diversity was introduced to the model first ( $\beta_{\text {time}}$ : Shannon index: $F_{1,9}=4.5, \mathrm{p}=0.06 ; \beta_{\text {space}}:$ Shannon index: $F_{1,9}=2.4, \mathrm{p}=0.152$, Table 3.1). No proportional change in the contribution of $\alpha-, \beta_{\text {time- }}$ or $\beta_{\text {space- }}$ to total $\gamma$-diversity within each forest stand was observed, neither for all tree species nor for beech alone (Appendix 3.4).

Community composition \& species richness of functional groups

Analysing all examined tree species, approximately one quarter of the sampled true bug species were associated with deciduous forest as habitat type (21 species, $27.6 \%$, Appendix 3.2). The rest of the species were more generalistic and did not prefer any particular habitat, (12 species, $15.8 \%$ ) or could be linked to other habitat types, most of them forest habitats but not strictly or primarily deciduous (43 species, 56.6\%). Species bound to deciduous forest also contributed the majority of individuals (914 individuals, $37.6 \%$ ), whereas only 162 individuals $(6.7 \%)$ were found to be habitat generalists. This pattern remained constant if beech alone was taken into account (10 (23.3\%) forest species, 208 (39.8\%) individuals; 7 (15.2 \%) habitat generalists, $32 \quad(6.1 \%)$ individuals, Appendix 3.2). 
Table 3.2. Multiple regression analyses of functional guild species richness for canopy true bugs captured on various tree species across a tree diversity gradient in the Hainich National Park. Values for beech alone are set in parentheses.

\begin{tabular}{|c|c|c|c|c|c|c|c|}
\hline \multirow[b]{2}{*}{$\begin{array}{l}\text { Response Variable } \\
\text { Species Richness of }\end{array}$} & \multirow[b]{2}{*}{ Effect } & \multicolumn{3}{|c|}{ Model A: } & \multicolumn{3}{|l|}{ Model B: } \\
\hline & & Multiple r2 & $F$ & $\mathrm{p}$ & Multiple r2 & $F$ & $\mathrm{p}$ \\
\hline \multirow[t]{2}{*}{ Habitat Generalists } & Abundance & & 10.64 (36.98) & $0.01(<0.001)$ & & $7.16(30.06)$ & $0.025(<0.001)$ \\
\hline & Shannon Index & $0.55(0.81)$ & $0.57(0.54)$ & $0.47(0.481)$ & $0.55(0.81)$ & $4.04(7.46)$ & $0.075(\mathbf{0 . 0 2 3})$ \\
\hline \multirow[t]{2}{*}{ Forest Species } & Abundance & & $86.42(5.7)$ & $<0.001(0.04)$ & & $7.1(2.36)$ & $\mathbf{0 . 0 2 6}(0.159)$ \\
\hline & Shannon Index & $0.91(0.39)$ & $3.03(<0.01)$ & $0.116(0.973)$ & $0.91(0.39)$ & 82.35 (3.34) & $<0.001(0.1)$ \\
\hline \multirow[t]{2}{*}{ Tree Layer Species } & Abundance & & $66.32(8.24)$ & $<0.001(0.018)$ & & $4.74(1.76)$ & $0.058(0.218)$ \\
\hline & Shannon Index & $0.88(0.48)$ & $2.3(0.19)$ & $0.164(0.674)$ & $0.88(0.48)$ & $63.88(6.68)$ & $<0.001(0.03)$ \\
\hline \multirow[t]{2}{*}{ Herb Layer Species } & Abundance & & $7.76(25.67)$ & $0.021(<0.001)$ & & $4.57(7.28)$ & $0.061(\mathbf{0 . 0 2 4})$ \\
\hline & Shannon Index & $0.46(0.75)$ & $<0.01(1.97)$ & $0.996(0.194)$ & $0.46(0.75)$ & $3.18(20.36)$ & 0.108 (0.001) \\
\hline \multirow[t]{2}{*}{ Predators } & Abundance & & $65.73(15.74)$ & $<0.001(0.003)$ & & $8.83(3.44)$ & $\mathbf{0 . 0 1 6}(0.097)$ \\
\hline & Shannon Index & $0.88(0.64)$ & $0.28(0.44)$ & $0.613(0.523)$ & $0.88(0.64)$ & $57.17(12.74)$ & $<0.001(0.006)$ \\
\hline \multirow[t]{2}{*}{ Herbivores } & Abundance & & $75.77(16.72)$ & $<0.001(0.003)$ & & $7.33(6.13)$ & $0.024(0.008)$ \\
\hline & Shannon Index & $0.91(0.66)$ & $14.81(0.67)$ & $\mathbf{0 . 0 0 4}(0.433)$ & $0.91(0.66)$ & $83.25(11.26)$ & $<0.001(0.035)$ \\
\hline
\end{tabular}


The number of forest species and habitat generalists found on various tree species increased across the tree diversity gradient (Fig. 3.3a), but both groups were again controlled by increased individual abundance in the most species rich forest stands compared to the simple beech stands. Forest species responded significantly to the tested variables (Shannon index: $F_{1,9}=82.35, \mathrm{p}<0.001$, Abundance: $F_{1,9}=7.1, \mathrm{p}=0.026$, Table 3.2), while a relationship of habitat generalists to tree diversity remained only marginally significant, even if tree diversity was introduced to the multiple regression model first (Shannon index: $F_{1,9}=4.04, \mathrm{p}=0.075$, Abundance: $F_{1,9}=7.16, \mathrm{p}=0.025$, Table 3.2). The opposite was found if beech alone was analysed (Fig. 3.3b). Here, forest species showed only a significant response to individual abundance if it was introduced to the model first, and no relationship with tree diversity at all (Shannon index: $F_{1,9}=3.34, \mathrm{p}=0.1$, Abundance: $F_{1,9}=2.36, \mathrm{p}=0.159$, Table 3.2), whereas the number of habitat generalists increased across the gradient (Shannon index: $F_{1,9}=7.46, \mathrm{p}$ $=0.023$, Abundance: $F_{1,9}=30.06, \mathrm{p}<0.001$, Table 3.2).

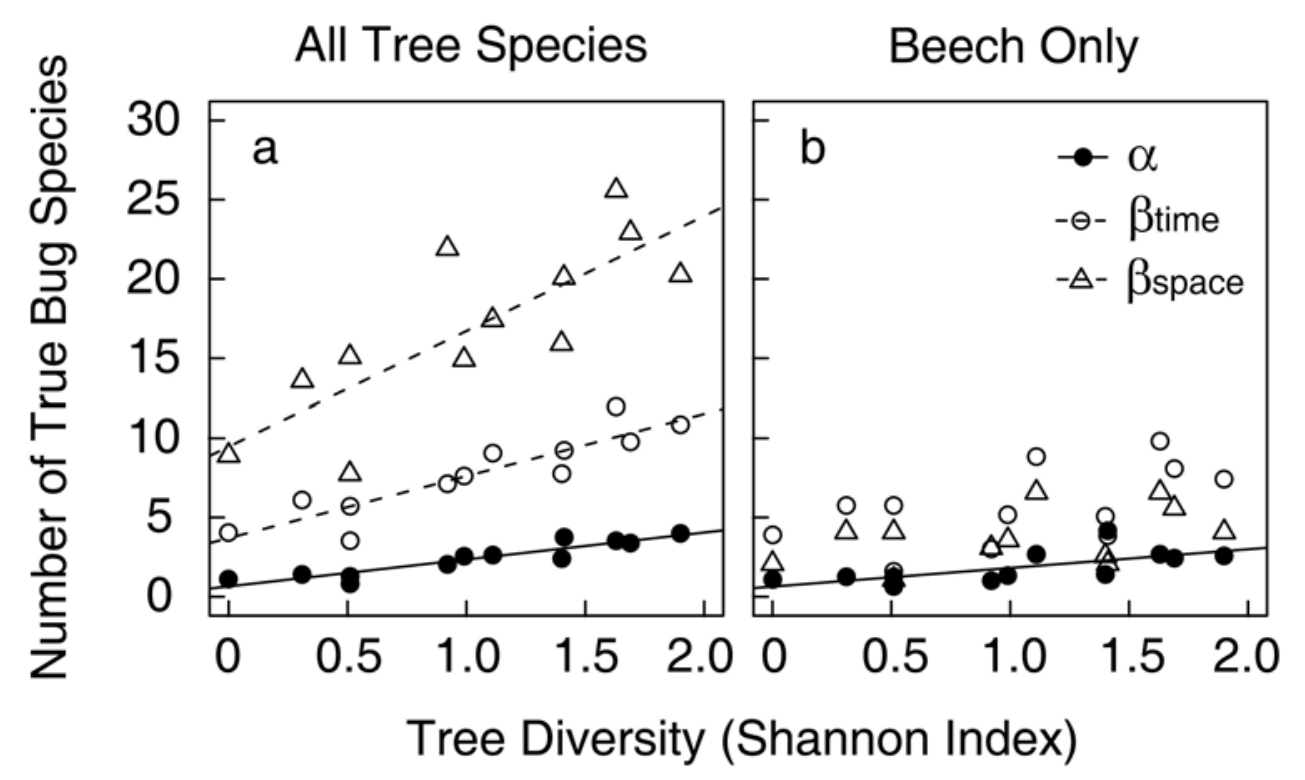

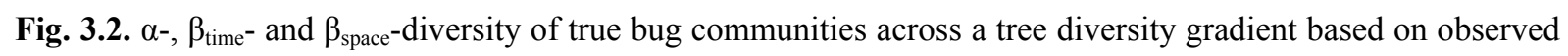
species richness per plot. a) All tree species sampled, regression slopes differ significantly (ANCOVA, $F_{2,30}=$ $7.11, \mathrm{p}=0.003), \mathrm{b})$ beech only.

Concerning stratification, the majority of species and individuals found on various tree species were found to be associated with the tree layer (42 species (55.3\%), 2139 individuals (88\%), Appendix 3.2), while only a small fraction was contributed to tourist species typically dwelling in the forest herb layer (13 species (17.1\%), 50 individuals (3.9\%), Appendix 3.2). Yet again, the gross proportions remained consistent if beech alone was considered (tree 
layer: 24 species $(55.8 \%), 448$ individuals (85.8\%), herb layer: 8 species (18.6\%), 20 individuals $(3.8 \%))$. Tree dwelling species showed a positive linear relationship to increased tree diversity (Fig. 3.3c), but only if tree diversity was introduced to the multiple regression model before controlling for individual abundance (Shannon index: $F_{1,9}=63.88, \mathrm{p}<0.001$, Abundance: $F_{1,9}=4.74, \mathrm{p}=0.058$, Table 3.2). Neither individual abundance nor tree diversity influenced species richness of herb layer species (Shannon index: $F_{1,9}=3.18, \mathrm{p}=0.108$, Abundance: $F_{1,9}=4.57, \mathrm{p}=0.061$, Table 3.2). If beech alone was considered, species richness of both tree layer dwelling species (Shannon index: $F_{1,9}=6.68, \mathrm{p}=0.03$, Abundance: $F_{1,9}=$ $1.76, \mathrm{p}=0.218$, Table 3.2) and herb layer dwelling species (Shannon index: $F_{1,9}=20.36, \mathrm{p}<$ 0.001, Abundance: $F_{1,9}=7.28, \mathrm{p}=0.024$, Table 3.2) increased across the gradient (Fig. 3.3d), but effects were only apparent when tree diversity entered the model first. Assorted to feeding guilds, the majority of species found on all trees was described as predatory according to the feeding ecology of adults (41 species, $53.9 \%$, Appendix 3.2). In contrast, only 13 (17.1\%) species were annotated as strictly herbivorous. By means of individual abundance, predators were also most common (2185 individuals, $89.9 \%$ ), whereas only 96 (3.9\%) of all individuals belonged to the herbivore guild. Communities on beech exhibited the same distribution of herbivores and predators (herbivores: 8 (18.6\%) species, 21 (4\%) individuals; predators: $21(48.8 \%)$ species, 449 (86\%) individuals, Appendix 3.2). The number of predators as well as of herbivores increased across the tree diversity gradient (Fig. 3.3e), but only species richness of herbivores showed a significant response to tree diversity after previously controlling for increased individual abundance (Abundance: $F_{1,9}=75.77, \mathrm{p}<$ 0.001, Shannon index: $F_{1,9}=14.81, \mathrm{p}=0.004$, Table 3.2). A similar pattern was found for species richness on beech alone (Fig. 3.3f), although here the herbivore guild did not respond to tree diversity if individual abundance was entered to the model first (Abundance: $F_{1,9}=$ 16.72, $\mathrm{p}=0.003$, Shannon index: $F_{1,9}=0.67, \mathrm{p}=0.433$, Table 3.2 ).

Most true bug species and individuals captured on various tree species exhibited an intermediate degree of host plant specialisation (22 (28.9 \%) species, 575 (23.7\%) individuals, Appendix 3.2). 12 (15.8\%) species were highly specialised on a single plant species or genus (441 (18.1\%) individuals), and 18 (23.7\%) species were not specialised at all (334 (13.7\%) individuals), thus being capable of dwelling on a variety of plant genera. Similar proportions of species and individuals were found on beech alone (5 (11.6\%) strict species, 37 (7.1\%) individuals; 9 (20.9\%) intermediate species, 145 (27.8\%) individuals; 15 (34.9\%) non-specialised species, 87 (16.7\%) individuals, Appendix 3.2). 


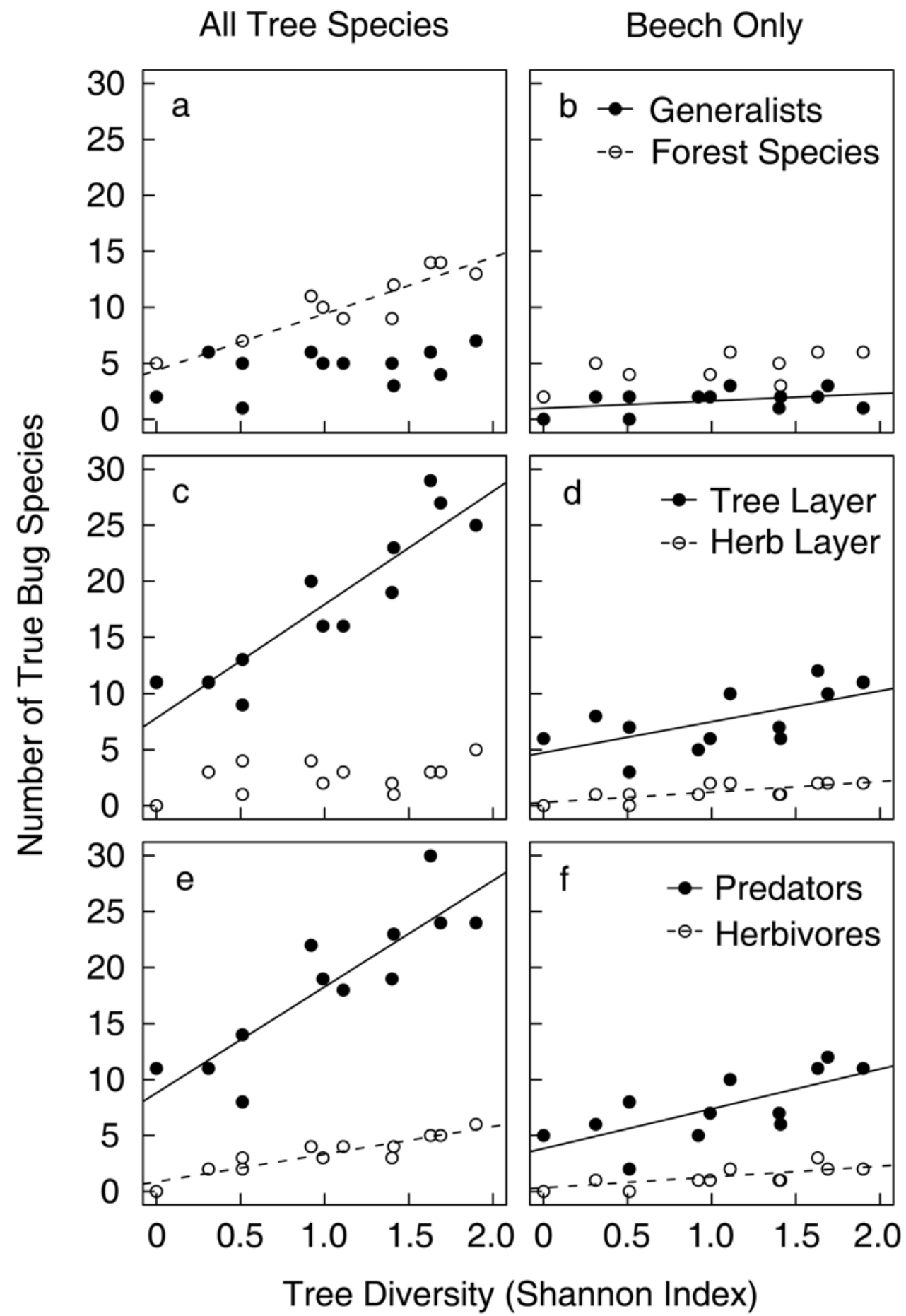

Fig. 3.3. Species richness of functional groups of true bugs across a tree diversity gradient on various tree species. a \& b) habitat specialisation, c \& d) stratification specialisation, e \& f) feeding guilds (a, c, e all tree species sampled, b, d, f beech only). 
Table 3.3. Multiple regression analyses of host plant and prey specialisation for canopy true bugs captured on various tree species across a tree diversity gradient in the Hainich National Park. Values for beech alone are set in parentheses. ${ }^{\mathrm{H}}$ host plant specialisation (food or habitat resource), ${ }^{\mathrm{P}}$ prey specialisation.

\begin{tabular}{|c|c|c|c|c|c|c|c|}
\hline & \multirow[b]{2}{*}{ Effect } & \multicolumn{3}{|l|}{ Model A: } & Shannon Index + Abundance & \multicolumn{2}{|c|}{ Model B: } \\
\hline $\begin{array}{l}\text { Response Variable } \\
\text { Species Richness of }\end{array}$ & & Multiple r2 & $F$ & $\mathrm{p}$ & Multiple r2 & $F$ & $\mathrm{p}$ \\
\hline \multirow[t]{2}{*}{ Strict $^{\mathrm{H}}$ Species } & Abundance & & $97.09(42.24)$ & $<0.001(<0.001)$ & & $9.39(19.47)$ & $0.013(0.002)$ \\
\hline & Shannon Index & $0.92(0.83)$ & $5.15(0.85)$ & $\mathbf{0 . 0 5}(0.379)$ & $0.92(0.83)$ & $92.85(23.63)$ & $<0.001(<0.001)$ \\
\hline \multirow[t]{2}{*}{ Intermediate $^{\mathrm{H}}$ Species } & Abundance & & $20.42(7.23)$ & $0.001(0.025)$ & & $0.93(8.46)$ & 0.359 (0.017) \\
\hline & Shannon Index & $0.72(0.5)$ & $2.56(1.87)$ & $0.143(0.205)$ & $0.72(0.5)$ & $22.05(0.64)$ & $\mathbf{0 . 0 0 1}(0.445)$ \\
\hline \multirow[t]{2}{*}{ Not Specialised ${ }^{\mathrm{H}}$ Species } & Abundance & & $36.05(7.52)$ & $<0.001(0.023)$ & & $3.24(3.94)$ & $0.105(0.078)$ \\
\hline & Shannon Index & $0.8(0.46)$ & $0.55(0.04)$ & $0.477(0.849)$ & $0.8(0.46)$ & $33.36(3.62)$ & $<\mathbf{0 . 0 0 1}(0.09)$ \\
\hline \multirow[t]{2}{*}{ Oligophagous ${ }^{\mathrm{P}}$ Species } & Abundance & & $43.8(19.37)$ & $<0.001(0.002)$ & & $2.66(9.42)$ & $0.138(\mathbf{0 . 0 1 3 )}$ \\
\hline & Shannon Index & $0.84(0.68)$ & $3.78(0.16)$ & $0.084(0.702)$ & $0.84(0.68)$ & $44.93(10.11)$ & $<0.001(0.011)$ \\
\hline \multirow[t]{2}{*}{ Polyphagous ${ }^{\mathrm{P}}$ Species } & Abundance & & $6.2(3.01)$ & $\mathbf{0 . 0 3 4}(0.117)$ & & $0.06(1.5)$ & $0.809(0.252)$ \\
\hline & Shannon Index & $0.48(0.29)$ & $2.01(0.72)$ & $0.19(0.418)$ & $0.48(0.29)$ & $8.15(2.24)$ & $\mathbf{0 . 0 1 9}(0.169)$ \\
\hline
\end{tabular}


In the analyses of various tree species combined, species richness in all groups increased across the tree diversity gradient (Fig. 3.4a), but only strictly specialised true bugs responded to tree diversity after previous control for increased individual abundance (Abundance: $F_{1,9}=$ 97.09, $\mathrm{p}<0.001$, Shannon index: $F_{1,9}=5.15, \mathrm{p}=0.05$, Table 3.3). Effects were similar but less significant if beech alone was considered (Fig. 3.4b). Even if tree diversity was introduced to the model first, intermediately specialised (Shannon index: $F_{1,9}=0.64, \mathrm{p}=$ 0.445, Abundance: $F_{1,9}=8.46, \mathrm{p}=0.017$, Table 3.3) and non-specialised species (Shannon index: $F_{1,9}=3.64, \mathrm{p}=0.09$, Abundance: $F_{1,9}=3.94, \mathrm{p}=0.078$, Table 3.3) did not respond at all, and only strictly specialised species were significantly affected by the tree diversity gradient (Shannon index: $F_{1,9}=23.63, \mathrm{p}<0.001$, Abundance: $F_{1,9}=19.47, \mathrm{p}=0.002$, Table $3.3)$.

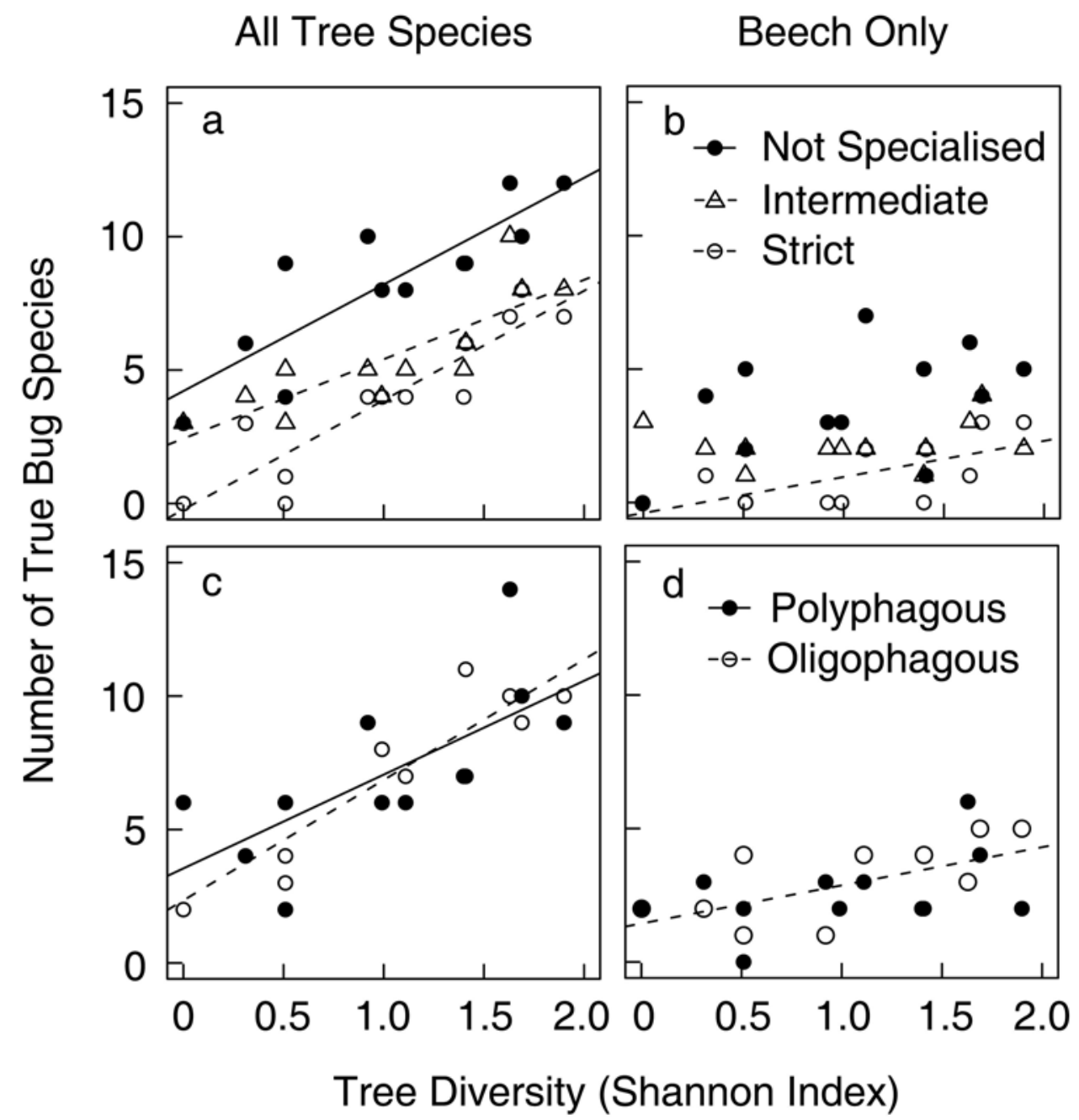

Fig. 3.4. Species richness of functional groups of true bugs across a tree diversity gradient on various tree species. a \& c) host plant specialisation, b \& d) prey specialisation, (a, c all tree species sampled, b, d beech only). 
No predatory true bug species were found to be strictly dependent on a single prey species. In contrast, most individuals found on various tree species were oligophagous with a specialisation on several prey taxa (14 species (34.1\%), 998 (45.7\%) individuals), or polyphagous and capable of feeding on high variety of prey (20 (48.8\%) species, 941 (43.1 $\%$ ) individuals, Appendix 3.2). Results were similar if beech alone was taken into account (8 polyphagous species (38.1\%), 204 individuals (45\%), 8 oligophagous species (38.1\%), 184 individuals (41\%), Appendix 3.2). For various tree species (Fig. 3.4c), both oligophagous true bug species richness (Shannon index: $F_{1,9}=44.93, \mathrm{p}<0.001$, Abundance: $F_{1,9}=2.66, \mathrm{p}=$ 0.138 , Table 3.3) and polyphagous true bug species richness were found to increase with the tree diversity gradient, but only if Shannon index was introduced to the model first (Shannon index: $F_{1,9}=8.15, \mathrm{p}=0.019$, Abundance: $F_{1,9}=0.06, \mathrm{p}=0.809$, Table 3.3). Considering beech alone (Fig. 3.4d), a similar response in oligophagous species was only found if tree diversity was introduced to the model first (Shannon index: $F_{1,9}=10.11, \mathrm{p}=0.011$, Abundance: $F_{1,9}=9.42, \mathrm{p}=0.013$, Table 3.3), but even then species richness of polygophageous species was not affected (Shannon index: $F_{1,9}=2.24, \mathrm{p} 0.169$, Abundance: $F_{1,9}=1.5, \mathrm{p}=0.252$, Table 3.3).

No differences or specific patterns in proportional species richness could be determined for any of the groupings or guilds within each forest stand across the gradient (Appendix $3.5 \&$ 3.6), neither if all tree species were analysed, nor beech alone.

\section{Discussion}

Overall $\gamma$-diversity of true bugs increased across the tree diversity gradient as hypothesised. However, rather than tree diversity as such, increased abundance of true bugs in the most species-rich forest stands turned out to primarily account for increased species richness of this taxon. The same pattern was exhibited by species richness in most functional guilds. When additive partitioning of true bug diversity was performed, $\alpha$-diversity also was primarily influenced by differences in true bug abundance rather than by tree diversity, and the mean number of species per tree and month was only slightly higher in the more diverse forest stands. Regression slopes were much steeper for spatiotemporal species turnover, and both $\beta_{\text {time }}$ and $\beta_{\text {space }}$ showed a strong response to tree diversity. $\beta$-diversity contributed roughly 90 $\%$ to total $\gamma$-diversity, which stresses the importance of species turnover between individual trees, tree species and months for diversity estimates. For a reliable determination of arthropod diversity across habitat gradients, sufficient sampling effort, both in space and time, 
is crucial (Tylianakis et al. 2005). As our results illustrate, focusing on a single tree species abundant in all stands (here beech) would have been vastly misleading, indicating that $\beta_{\text {time }}$ contributes more to true bug diversity than $\beta_{\text {space, }}$ with neither of them responding to tree diversity. This pattern was reversed if simply more replicates (here trees) or more tree species were included in the analyses. In particular, spatial turnover between tree individuals of the same species was surprisingly high, which only became apparent when six instead of two beech trees were compared in the most simple forest stands. The latter result is unexpected, because forest stands primarily consisting of beech on first sight appear to be a much more continuous habitat compared to forest stands consisting of various tree species. Hence we presumed that spatial turnover would play a minor role in the more simple stands. Although plant identity and diversity strongly promotes species richness of associated arthropods (Siemann 1998, Siemann et al. 1998, Brändle et al. 2001, Haddad et al. 2001), spatial turnover seems to be controlled by individual differences among trees of the same species as well. Lindo \& Winchester (2008) found high species turnover among conspecific trees for oribatid mites, and one explanation for this was limitated dispersal due to low mobility of these organisms. In contrast to mites, true bugs are a lot more mobile, and in simple beech

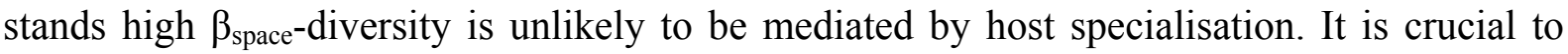
investigate how microhabitat conditions of individual trees differ and whether this leads to a patchy distribution of true bug species, which in turn increases $\beta_{\text {space-diversity even among }}$ conspecific individuals, thereby mediating overall $\gamma$-diversity.

With tree diversity a priori set as controlling variable, how can observed differences in true bug richness across the gradient be explained? In our study, canopy tree diversity determined various biotic and abiotic plot characteristics and structural parameters (e.g. plant area index, stem density), which were highly correlated with the Shannon index of the sampled forest stands. Not only the number of true bug species was increased with increasing tree diversity, but also the number of individuals captured in the most diverse forest stand was enhanced and highly correlated with the Shannon index. Highly structured vegetation, such as a high variety of tree species with different traits, provides a high variety of resources and sites for reproduction, colonisation, and overwintering (Lawton 1983). Compared with simple beech stands, diverse forest stands most likely offer a greater array of niches, which in turn can enhance true bug abundance and with it diversity.

The increase in structural complexity mediated by tree diversity resulted in greater habitat heterogeneity, which has been suggested as an overarching factor determining animal species richness regardless of taxon or habitat type (Tews et al. 2004). Additionally, different tree 
species themselves differ in architecture, bark texture, branching complexity, leave abundance and other features. Hence, micro-climate, shelter-availability and accessibility differs for various tree species, and the resulting niches can support different sets of arthropod abundance and diversity (Lawton 1983, Southwood et al. 1982, Halaj et al. 1998, Halaj et al. 2000, Goßner \& Ammer 2006). Although most species captured were not dependend on a particular host plant as habitat or food resource, the number of species with intermediate or strict host affiliation strongly increased across the gradient, indicating the importance of availability of certain tree or plant species and the associated structures. If the latter are lacking, a high proportion of species will vanish.

Some authors could not find a relationship of insect richness and habitat complexity when flight interception traps in forests were used (Lassau et al. 2005), but this might be due spatial (low number of traps, restriction to understory) and temporal sampling bias. For beetles, Lassau et al. (2005) suggest easier accessibility of the traps in more simple habitats as reason for the lack of differences in species richness compared to complex habitats. Sampling bias due to more barrier-free flight-paths might explain some of the species richness we observed by sampling oak and maple, because the crowns of these trees appeared to be more open in contrast to other tree species. Nevertheless, we can diminish this objection by comparing beech alone, which had similarly structured crowns on all sampled plots, but still reflected differences of true bug richness across the tree diversity gradient. Moreover, taxa might respond differently to gradients of plant diversity and habitat structure in general, which highlights the necessity to include previously neglected taxa like true bugs in biodiversity survey.

Concerning functional guilds, forest species, and in particular, tree-dwelling species, were most abundant in the traps and distinctly responded to the tree diversity gradient, whereas habitat generalists were less species-rich and showed a weaker response. This illustrates that true bugs in deciduous forest show a distinct stratification in species distribution. Further research is needed to investigate community patterns in other strata and how they compare to patterns observed in the canopy. Abundance and species richness of predators and herbivores would be of particular interest with regard to ecosystem functioning (herbivory and biocontrol) in the forest understory.

In the canopy, predators were generally more species-rich compared to herbivores, and species richness of predators also increased with the tree diversity gradient. Predator richness of true bugs has been linked to vegetation structure (Zurbrügg \& Frank 2006), and intra-guild predation might decrease in highly structured vegetation due to a greater availability of 
refuges (Morris 2000, Finke \& Denno 2002, Corkum \& Cronin 2004). This mechanism only holds true for generalist predators, and is legitimate to be applied here since none of the species observed was higly specialised or restricted to a single prey species. For the same reason, increased habitat complexity positively affects individual abundance of predators (Langellotto \& Denno 2004), but this does not necessarily mean that as a cascading effect, herbivores become less abundant and species-rich. As illustrated by the increase in herbivore species richness across the tree diversity gradient, the lower trophic levels might also profit from resource availability and shelter, which might limit top-down control (Sanders et al. 2008).

We conclude that the additive partitioning of diversity across space and time is a necessary tool to evaluate diversity of insect communites, in particular if it is desired to determine what contributes most to the total observed diversity within one habitat. Here, species turnover $\beta_{\text {space }}$ between tree species and tree individuals has been shown to be of great importance for increasing diversity of true bug communities, which has direct implications for forest management. Simple beech stands alone only insufficiently conserve regional species richness of true bugs, which can only be maintained by a mix of different tree species and individuals. This ensures habitat heterogeneity and increased microhabitat abundance supporting a higher load of true bug individuals, and with it a greater number of species. Tree diversity, by means of species identity and individual identity, hence indirectly controls several factors that in turn influence species richness of true bug communities. We show that species rich deciduous forests are of particular importance for forest dependent true bug species, which rely on certain tree species as food or reproductive resource. The current intensification of forestry leads to increased habitat loss, habitat fragmentation and reduced habitat complexity (Kouki et al. 2001, Larsson \& Danell 2001, Hirao et al. 2007, Müller \& Goßner 2007), eventually resulting in forests unsuitable to maintain a true bug community dependend on a diverse mix of deciduous trees and niches.

\section{Acknowledgements}

This study obtained financial support by the DFG [German Research Foundation] within the Research Training Group „Graduiertenkolleg 1086: The role of biodiversity for biogeochemical cycles and biotic interactions in temperate deciduous forests“. We highly acknowledge Christoph Leuschner, Frank Thomas, Hermann Jungkunst and Stefan Fleck for coordination of the group and also thank Mascha Brauns, Karl M. Daenner, Mareen Gollnow, 
Inga Krämer and Inga Schmidt for providing data on stand characteristics. Our special thanks go to Martin Erren, Tobias Gebauer, Susanne Schiele and Ulrich Simon for assistance with field work.

\section{References}

Clough, Y., Holzschuh, A., Gabriel, D., Purtauf, T., Kleijn, D., Kruess, A., Steffan-Dewenter, I., \& Tscharntke, T. (2007) Alpha and Beta Diversity of Arthropods and Plants in Organically and Conventionally Managed Wheat Fields. Journal of Applied Ecology 44, 804-812.

Colwell, R.K. (2006) EstimateS: Statistical Estimation of Species Richness and Shared Species from Samples. Version 7.5. User's guide and application published at http://purl.oclc.org/estimates.

Corkum, L.D. \& Cronin, D.J. (2004) Habitat Complexity Reduces Aggression and Enhances Consumption in Crayfish. Journal of Ethology 22, 23-27.

Crist, T.O., Veech, J.A., Gering, J.C., \& Summerville, K.S. (2003) Partitioning Species Diversity Across Landscapes and Regions: a Hierarchical Analysis of Alpha, Beta, and Gamma Diversity. American Naturalist 162, 734-743.

Duelli, P. \& Obrist, M.K. (1998) In Search of the Best Correlates for Local Organismal Biodiversity in Cultivated Areas. Biodiversity and Conservation 7, 297-309.

Fauvel, G. (1999) Diversity of Heteroptera in Agroecosystems: Role of Sustainability and Bioindication. Agriculture Ecosystems \& Environment 74, 275-303.

Finke, D.L. \& Denno, R.F. (2002) Intraguild Predation Diminished in Complex-Structured Vegetation: Implications for Prey Suppression. Ecology 83, 643-652.

Goßner, M. \& Ammer, U. (2006) The Effects of Douglas-Fir on Tree-Specific Arthropod Communities in Mixed Species Stands With European Beech and Norway Spruce. European Journal of Forest Research 125, 221-235.

Goßner, M., Liston, A., \& Spaeth, J. (2007) Sawflies in the Crowns of Native and Exotic Trees, Sampled With Flight-Interception Traps in Southern Germany (Hymenoptera : Symphyta). Entomologia Generalis 30, 273-282.

Goßner, M. (2008) Heteroptera (Insecta: Hemiptera) Communities in Tree Crowns of Beech, 
Oak, and Spruce in Managed Forests- Diversity, Seasonality, Guild Structure, and Tree Specificity. Structure, Diversity and Functional Aspects of Arthropod Fauna in Central European Canopies (ed. by Floren A. and J. Schmidl), bioform, Heroldsberg.

Haddad, N.M., Tilman, D., Haarstad, J., Ritchie, M., \& Knops, J.M.H. (2001) Contrasting Effects of Plant Richness and Composition on Insect Communities: a Field Experiment. The American Naturalist 158, 17-35.

Halaj, J., Ross, D.W., \& Moldenke, A.R. (1998) Habitat Structure and Prey Availability as Predictors of the Abundance and Community Organisation of Spiders in Western Oregon Forest Canopies. Journal of Arachnology 26, 203-220.

Halaj, J., Ross, D.W., \& Moldenke, A.R. (2000) Importance of Habitat Structure to the Arthropod Food-Web in Douglas-Fir Canopies. Oikos 90, 139-152.

Hamer, K.C., Hill, J.K., Benedick, S., Mustaffa, N., Sherratt, T.N., Maryati, M., \& Chey, V.K. (2003) Ecology of Butterflies in Natural and Selectively Logged Forests of Northern Borneo: the Importance of Habitat Heterogeneity. Journal of Applied Ecology 40, 150-162.

Hansen, R.A. (2000) Effects of Habitat Complexity and Composition on a Diverse Litter Microarthropod Assemblage. Ecology 81, 1120-1132.

Hirao, T., Murakami, M., Kashizaki, A., \& Tanabe, S.I. (2007) Additive Apportioning of Lepidopteran and Coleopteran Species Diversity Across Spatial and Temporal Scales in a Cool-Temperate Deciduous Forest in Japan. Ecological Entomology 32, 627-636.

Humphrey, J.W., Hawes, C., Peace, A.J., Ferris-Kaan, R., \& Jukes, M.R. (1999) Relationships Between Insect Diversity and Habitat Characteristics in Plantation Forests. Forest Ecology and Management 113, 11-21.

Kouki, J., Lofman, S., Martikainen, P., Rouvinen, S., \& Uotila, A. (2001) Forest Fragmentation in Fennoscandia: Linking Habitat Requirements of Wood-Associated Threatened Species to Landscape and Habitat Changes. Scandinavian Journal of Forest Research 27-37.

Lande, R. (1996) Statistics and Partitioning of Species Diversity, and Similarity Among Multiple Communities. Oikos 76, 5-13.

Langellotto, G.A. \& Denno, R.F. (2004) Responses of Invertebrate Natural Enemies to 
Complex-Structured Habitats: a Meta-Analytical Synthesis. Oecologia 139, 1-10.

Larsson, S. \& Danell, K. (2001) Science and the Management of Boreal Forest Biodiversity. Scandinavian Journal of Forest Research 5-9.

Lassau, S.A. \& Hochuli, D.F. (2008) Testing Predictions of Beetle Community Patterns Derived Empirically Using Remote Sensing. Diversity and Distributions 14, 138-147.

Lassau, S.A., Hochuli, D.F., Cassis, G., \& Reid, C.A.M. (2005) Effects of Habitat Complexity on Forest Beetle Diversity: Do Functional Groups Respond Consistently? Diversity and Distributions 11, 73-82.

Lawton, J.H. (1983) Plant Architecture and the Diversity of Phytophagous Insects. Annual Review of Entomology 28, 23-29.

Leuschner, C., Meier, I.C., \& Hertel, D. (2006) On the Niche Breadth of Fagus sylvatica: Soil Nutrient Status in 50 Central European Beech Stands on a Broad Range of Bedrock Types. Annals of Forest Science 63, 355-368.

Lindo, Z. \& Winchester, N.N. (2008) Scale Dependent Diversity Patterns in Arboreal and Terrestrial Oribatid Mite (Acari : Oribatida) Communities. Ecography 31, 53-60.

Morris, M.G. (2000) The Effects of Structure and Its Dynamics on the Ecology and Conservation of Arthropods in British Grasslands. Biological Conservation 95, 129-142.

Müller, J., Bussler, H., \& Kneib, T. (2008) Saproxylic Beetle Assemblages Related to Silvicultural Management Intensity and Stand Structures in a Beech Forest in Southern Germany. Journal of Insect Conservation 12, 107-124.

Müller, J. \& Goßner, M. (2007) Single Host Trees in a Closed Forest Canopy Matrix: a Highly Fragmented Landscape? Journal of Applied Entomology 131, 613-620.

Ozanne, M.P. (1996) The Arthropod Communities of Coniferous Forest Trees. Selbyana 17, 43-49.

Polhemus, J.T. \& Polhemus, D.A. (2008) Global Diversity of True Bugs (Heteroptera : Insecta) in Freshwater. Hydrobiologia 595, 379-391.

Rabitsch, W., Goßner, M., Dorow, W.H.O., \& Melber, A. (in press) Ökologie der Wanzen 
Deutschlands, Österreichs und der Schweiz. - Beiträge zur bayerischen Entomofaunistik Supplementband 1 .

Sanders, D., Nickel, H., Grutzner, T., \& Platner, C. (2008) Habitat Structure Mediates TopDown Effects of Spiders and Ants on Herbivores. Basic and Applied Ecology 9, 152-160.

Siemann, E. (1998) Experimental Tests of Effects of Plant Productivity and Diversity on Grassland Arthropod Diversity. Ecology 79, 2057-2070.

Siemann, E., Tilman, D., Haarstad, J., \& Ritchie, M. (1998) Experimental Tests of the Dependence of Arthropod Diversity on Plant Diversity. The American Naturalist 152, 738750.

Southwood, T.R.E., Moran, V.C., \& Kennedy, C.E.J. (1982) The Richness, Abundance and Biomass of the Arthropod Communities on Trees. Journal of Animal Ecology 51, 635-649.

Southwood, T.R.E., Wint, G.R.W., Kennedy, C.E.J., \& Greenwood, S.R. (2005) The Composition of the Arthropod Fauna of the Canopies of Some Species of Oak (Quercus). Eur. J. Entomol. 102, 65-72.

Stork, N.E. (1988) Insect Diversity: Facts, Fiction and Speculation. Biological Journal of the Linnean Society 35, 321-337.

Tews, J., Brose, U., Grimm, V., Tielborger, K., Wichmann, M.C., Schwager, M., \& Jeltsch, F. (2004) Animal Species Diversity Driven by Habitat Heterogeneity/Diversity: the Importance of Keystone Structures. Journal of Biogeography 31, 79-92.

Thunes, K.H., Skarveit, J., \& Gjerde, I. (2003) The Canopy Arthropods of Old and Mature Pine Pinus sylvestris in Norway. Ecography 26, 490-502.

Tylianakis, J.M., Klein, A.M., Lozada, T., \& Tscharntke, T. (2006) Spatial Scale of Observation Affects Alpha, Beta and Gamma Diversity of Cavity-Nesting Bees and Wasps Across a Tropical Land-Use Gradient. Journal of Biogeography 33, 1295-1304.

Tylianakis, J.M., Klein, A.M., \& Tscharntke, T. (2005) Spatiotemporal Variation in the Diversity of Hymenoptera Across a Tropical Habitat Gradient. Ecology 86, 3296-3302.

Ulyshen, M.D. \& Hanula, J.L. (2007) A Comparison of the Beetle (Coleoptera) Fauna Captured at Two Heights Above the Ground in a North American Temperate Deciduous 
Forest. American Midland Naturalist 158, 260-278.

Veech, J.A., Summerville, K.S., Crist, T.O., \& Gering, J.C. (2002) The Additive Partitioning of Species Diversity: Recent Revival of an Old Idea. Oikos 99, 3-9.

Wachmann, E., Melber, A., \& Deckert, J. (2004) Wanzen Band 2 Goecke \& Evers, Keltern.

Zurbrügg, C. \& Frank, T. (2006) Factors Influencing Bug Diversity (Insecta : Heteroptera) in Semi-Natural Habitats. Biodiversity and Conservation 15, 275-294. 


\section{Appendix 3.1}

Tree diversity of 12 deciduous forest stands in the Hainich National Park and sampling success of local true bug communities. Stands ranking was based on increasing Shannon indices (tree diversity), rank 3 was assigned twice due to equal indices.

\begin{tabular}{lllll}
\hline $\begin{array}{l}\text { Shannon } \\
\text { Index }\end{array}$ & $\begin{array}{l}\text { Stand } \\
\text { Ranking }\end{array}$ & \% Beech & \% Lime & $\begin{array}{l}\text { Sampling } \\
\text { Success }\end{array}$ \\
\hline 0 & 1 & 100 & 0 & 70.6 \\
0.31 & 2 & 93.5 & 2.8 & 73.7 \\
0.51 & $3 \mathrm{a}$ & 83.3 & 0 & 82.8 \\
0.51 & $3 \mathrm{~b}$ & 87.5 & 3.6 & 63.8 \\
0.92 & 4 & 73.7 & 10.8 & 65 \\
0.99 & 5 & 59.4 & 2.3 & 73.2 \\
1.11 & 6 & 60.6 & 12.1 & 79.5 \\
1.4 & 7 & 47.7 & 33 & 70.6 \\
1.41 & 8 & 41.9 & 34.2 & 70 \\
1.63 & 9 & 2.4 & 63.9 & 75.5 \\
1.69 & 10 & 3.1 & 67.4 & 73 \\
1.9 & 11 & 13.2 & 37.9 & 76.4 \\
\hline
\end{tabular}




\section{Appendix 3.2}

Species list of true bugs collected on various tree species in the canopy of forest stands across a tree diversity gradient in the Hainich National Park. Annotations to ecology based on Rabitsch et al. (2008). ${ }^{\mathrm{D}}$ deciduous, ${ }^{\mathrm{H}}$ host plant specialisation (food or habitat resource), ${ }^{\mathrm{P}}$ prey specialisation, ${ }^{\mathrm{V}}$ various tree species, ${ }^{\mathrm{B}}$ beech only, ${ }^{*}$ because mainly adults were captured with flight interception traps, feeding guild classification is based on adult diet.

\begin{tabular}{|c|c|c|c|c|c|c|c|}
\hline Species & Habitat & Stratum & $\begin{array}{l}\text { Feeding } \\
\text { Guild* }\end{array}$ & Specialisation $^{\mathrm{H}}$ & Specialisation $^{\mathrm{P}}$ & $\mathrm{n}^{\mathrm{V}}$ & $\mathrm{n}^{\mathrm{B}}$ \\
\hline Acanthosoma haemorrhoidale & other & tree layer & other & not specialised & unknown & 4 & 1 \\
\hline Acanthosomatidae sp. & unknown & unknown & unknown & unknown & unknown & 1 & 1 \\
\hline Anthocoridae sp. & unknown & unknown & unknown & unknown & unknown & 5 & 2 \\
\hline Anthocoris amplicollis & forest $^{\mathrm{D}}$ & other & carnivor & other & oligophagous & 2 & 0 \\
\hline Anthocoris confusus & forest $^{\mathrm{D}}$ & tree layer & predator & other & oligophagous & 90 & 21 \\
\hline Anthocoris nemoralis & forest $^{\mathrm{D}}$ & tree layer & predator & other & polyphagous & 5 & 1 \\
\hline Berytinus minor & other & other & other & strict & unknown & 1 & 0 \\
\hline Blepharidopterus angulatus & other & tree layer & other & intermediate & polyphagous & 2 & 1 \\
\hline Campyloneura virgula & forest $^{\mathrm{D}}$ & tree layer & predator & not specialised & polyphagous & 41 & 10 \\
\hline Carpocoris purpureipennis & other & herb layer & other & not specialised & unknown & 1 & 1 \\
\hline Closterotomus biclavatus & other & other & predator & not specialised & other & 8 & 2 \\
\hline Cremnocephalus alpestris & other & tree layer & predator & intermediate & oligophagous & 1 & 0 \\
\hline Cyllecoris histrionius & forest $^{\mathrm{D}}$ & tree layer & predator & strict & polyphagous & 1 & 0 \\
\hline Deraeocoris lutescens & other & tree layer & predator & other & polyphagous & 708 & 171 \\
\hline Deraeocoris trifasciatus & forest $^{\mathrm{D}}$ & tree layer & predator & not specialised & polyphagous & 3 & 0 \\
\hline Dicranocephalus agilis & other & herb layer & herbivore & intermediate & unknown & 1 & 1 \\
\hline Dolycoris baccarum & no preference & herb layer & herbivore & not specialised & unknown & 12 & 3 \\
\hline Dryophilocoris flavoquadrimaculatus & other & tree layer & predator & strict & polyphagous & 3 & 0 \\
\hline Elasmostethus interstinctus & other & tree layer & other & intermediate & unknown & 1 & 1 \\
\hline Elasmostethus minor & other & tree layer & other & strict & unknown & 1 & 1 \\
\hline Eurydema oleracea & no preference & herb layer & herbivore & intermediate & unknown & 1 & 0 \\
\hline Harpocera thoracica & other & tree layer & predator & strict & oligophagous & 203 & 9 \\
\hline Himacerus apterus & no preference & other & predator & unknown & polyphagous & 7 & 0 \\
\hline Isometopus intrusus & other & tree layer & predator & not specialised & polyphagous & 5 & 0 \\
\hline Kleidocerys resedae & other & tree layer & other & intermediate & unknown & 52 & 14 \\
\hline Loricula elegantula & other & tree layer & predator & unknown & polyphagous & 6 & 5 \\
\hline Lygocoris contaminatus & other & tree layer & herbivore & intermediate & unknown & 4 & 0 \\
\hline Lygocoris viridis & other & tree layer & predator & intermediate & unknown & 7 & 0 \\
\hline Lygus pratensis & no preference & herb layer & herbivore & not specialised & unknown & 19 & 10 \\
\hline Mermitelocerus schmidtii & other & tree layer & predator & intermediate & polyphagous & 20 & 1 \\
\hline Mirinae sp. & unknown & unknown & unknown & unknown & unknown & 1 & 1 \\
\hline Miris striatus & other & tree layer & predator & not specialised & polyphagous & 12 & 1 \\
\hline Nabis pseudoferus & other & other & predator & unknown & unknown & 70 & 23 \\
\hline Notostira erratica & other & herb layer & other & intermediate & unknown & 1 & 1 \\
\hline Orius horvathi & no preference & other & predator & unknown & oligophagous & 27 & 2 \\
\hline Orius laticollis & other & other & predator & other & oligophagous & 4 & 0 \\
\hline Orius majusculus & other & other & predator & unknown & polyphagous & 1 & 0 \\
\hline Orius minutus/vicinus & no preference & other & predator & unknown & polyphagous & 77 & 13 \\
\hline Orthops basalis & no preference & herb layer & herbivore & intermediate & unknown & 1 & 0 \\
\hline Orthops kalmii & no preference & herb layer & herbivore & intermediate & unknown & 1 & 1 \\
\hline Orthotylinae sp. & unknown & unknown & unknown & unknown & unknown & 5 & 0 \\
\hline
\end{tabular}




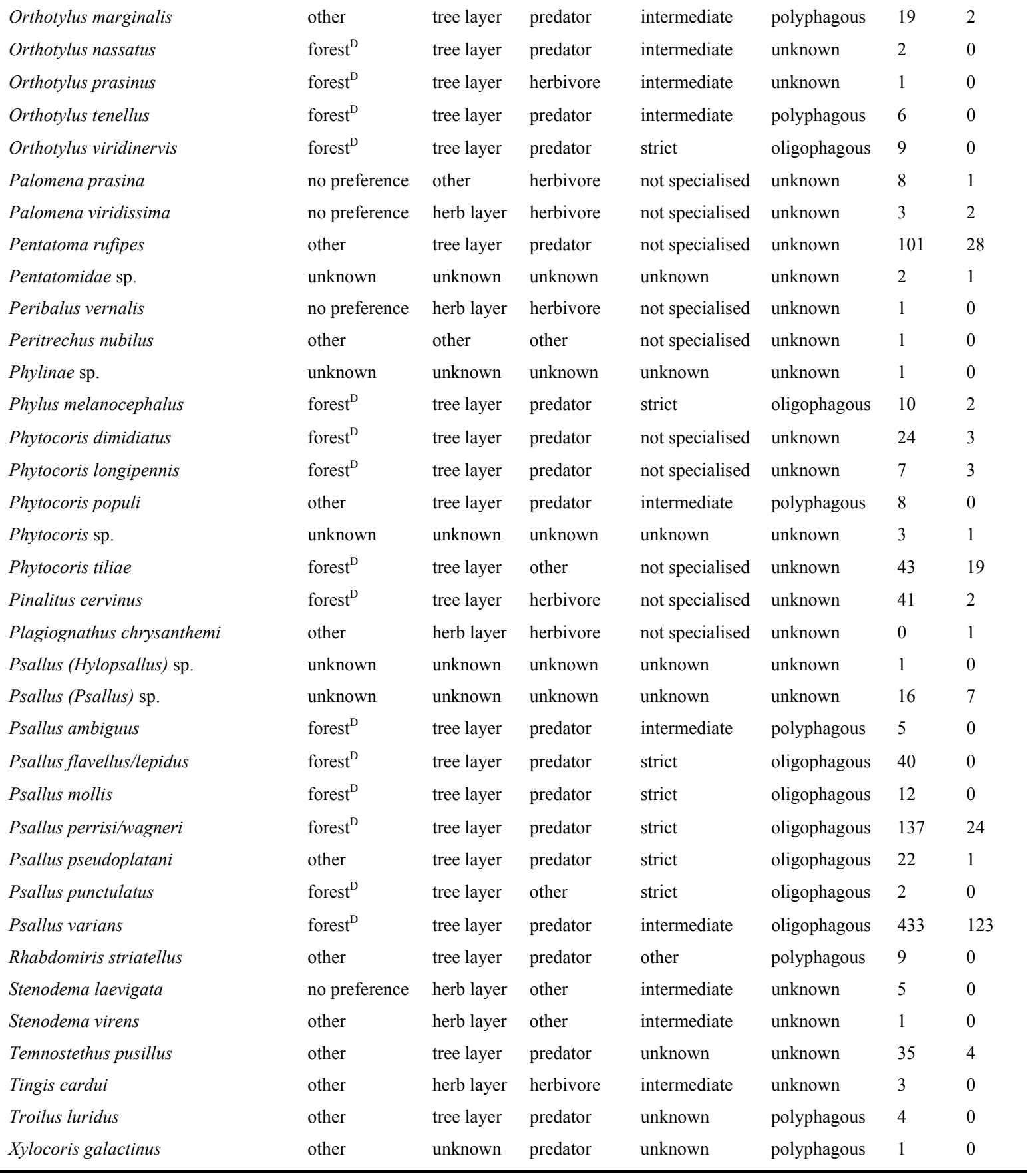




\section{Appendix 3.3}

Spearman rank correlations of untransformed response (true bug species richness) and explanatory variables.

\begin{tabular}{|c|c|c|c|c|c|c|c|c|c|c|}
\hline & $\begin{array}{l}\text { True Bug } \\
\text { Richness }\end{array}$ & $\begin{array}{l}\text { True Bug } \\
\text { Abundance }\end{array}$ & $\begin{array}{l}\text { Shannon } \\
\text { Index Trees }\end{array}$ & Stand Age & $\begin{array}{l}\text { Stem } \\
\text { Density }\end{array}$ & Tree Height & Crown Area & $\begin{array}{l}\text { Plant } \\
\text { Index }\end{array}$ & Area & $\begin{array}{l}\text { Deadwood } \\
\mathrm{m}^{3} / \mathrm{m}^{2}\end{array}$ \\
\hline True Bug Richness & 1 & & & & & & & & & \\
\hline True Bug Abundance & 0.89 & 1 & & & & & & & & \\
\hline Shannon Index Trees & 0.88 & 0.91 & 1 & & & & & & & \\
\hline Stand Age & -0.81 & -0.77 & -0.81 & 1 & & & & & & \\
\hline Stem Density & 0.45 & 0.23 & 0.34 & -0.72 & 1 & & & & & \\
\hline Tree Height & -0.8 & -0.79 & -0.78 & 0.95 & -0.63 & 1 & & & & \\
\hline Crown Area & -0.54 & -0.36 & -0.49 & 0.84 & -0.92 & 0.69 & 1 & & & \\
\hline Plant Area Index & 0.51 & 0.62 & 0.65 & -0.75 & 0.46 & -0.69 & -0.6 & 1 & & \\
\hline Deadwood $\mathrm{m}^{3} / \mathrm{m}^{2}$ & -0.57 & -0.45 & -0.36 & 0.66 & -0.54 & 0.77 & 0.51 & -0.21 & & 1 \\
\hline
\end{tabular}




\section{Appendix 3.4}

Relative contributions of $\alpha$ - and $\beta$-diversity to true bug species richness of communities sampled on various tree species in 12 forest plots across a tree diversity gradient. Values for beech alone are set in parentheses.

\begin{tabular}{llll}
\hline Forest Stand & $\% \alpha$ & $\% \beta_{\text {time }}$ & $\% \beta_{\text {space }}$ \\
\hline 1 & $8(15.5)$ & $28.9(56)$ & $63.1(28.6)$ \\
2 & $6.6(11.4)$ & $29.1(52.3)$ & $64.3(36.4)$ \\
$3 \mathrm{a}$ & $6.7(13.9)$ & $29.4(52.8)$ & $63.9(33.3)$ \\
$3 \mathrm{~b}$ & $5.8(11.4)$ & $26(52.3)$ & $68.2(36.4)$ \\
4 & $6.5(14.3)$ & $23(42.9)$ & $70.4(42.9)$ \\
5 & $10.2(13.3)$ & $30.4(51.7)$ & $59.3(35)$ \\
6 & $9(14.8)$ & $31.2(49.1)$ & $59.8(36.1)$ \\
7 & $9.2(15.7)$ & $29.9(56.5)$ & $60.9(27.8)$ \\
8 & $11.4(41.7)$ & $28(38.3)$ & $60.6(20)$ \\
9 & $8.6(14)$ & $29.2(51.8)$ & $62.2(34.2)$ \\
10 & $9.4(15.1)$ & $27.2(50.5)$ & $63.4(34.4)$ \\
11 & $11.4(18.5)$ & $31(53)$ & $57.6(28.6)$ \\
\hline
\end{tabular}

\section{Appendix 3.5}

Relative contributions of species abundance to functional guilds for canopy true bugs captured on various tree species across a tree diversity gradient in the Hainich National Park. Values for beech are set in parentheses.

\begin{tabular}{|c|c|c|c|c|c|c|}
\hline $\begin{array}{l}\text { Forest } \\
\text { Stand }\end{array}$ & $\begin{array}{l}\text { \% Habitat } \\
\text { Generalists }\end{array}$ & $\begin{array}{l}\% \quad \text { Forest } \\
\text { Species }\end{array}$ & $\begin{array}{l}\text { Tree Layer } \\
\text { Species }\end{array}$ & $\begin{array}{l}\text { Herb Layer } \\
\text { Species }\end{array}$ & Predators & Herbivores \\
\hline 1 & $14.3(0)$ & $35.7(28.6)$ & $78.6(85.7)$ & $0(0)$ & $78.6(71.4)$ & $0(0)$ \\
\hline 2 & $28.6(18.2)$ & $28.6(45.5)$ & $52.4(72.7)$ & $14.3(9.1)$ & $52.4(54.4)$ & $9.5(9.1)$ \\
\hline $3 a$ & $8.3(0)$ & $41.7(66.7)$ & $75(100)$ & $8.3(0)$ & $66.7(66.7)$ & $16.7(0)$ \\
\hline $3 b$ & $22.7(18.2)$ & $31.8(36.4)$ & $59.1(63.6)$ & $18.2(9.1)$ & $63.6(72.7)$ & $13.6(18.2)$ \\
\hline 4 & $19.4(28.6)$ & $35.5(28.6)$ & $64.5(71.4)$ & $12.9(14.3)$ & $71(71.4)$ & $12.9(14.3)$ \\
\hline 5 & $20(20)$ & $40(40)$ & $64(60)$ & $8(20)$ & $76(70)$ & $12(10)$ \\
\hline 6 & $17.2(16.7)$ & $31(33.3)$ & $55.2(55.6)$ & $10.3(11.1)$ & $62.1(55.6)$ & $13.8(11.1)$ \\
\hline 7 & $19.2(11.1)$ & $34.6(55.6)$ & $73.1(77.8)$ & $7.7(11.1)$ & $73.1(77.8)$ & $11.5(11.1)$ \\
\hline 8 & $9.1(20)$ & $36.4(30)$ & $69.7(60)$ & $3(10)$ & $69.7(60)$ & $12.1(10)$ \\
\hline 9 & $14.6(10.5)$ & $34.1(31.6)$ & $70.7(63.2)$ & $7.3(10.5)$ & $73.2(57.9)$ & $12.2(15.8)$ \\
\hline 10 & $11.1(18.8)$ & $38.9(18.8)$ & $75(62.5)$ & $8.3(12.5)$ & $66.7(75)$ & $13.9(12.5)$ \\
\hline 11 & $20(7.1)$ & $37.1(42.9)$ & $71.4(78.6)$ & $14.3(14.3)$ & $68.6(78.6)$ & $17.1(14.3)$ \\
\hline
\end{tabular}




\section{Appendix 3.6}

Relative contributions of species abundance to host plant and prey specialisation for canopy true bugs captured on various tree species across a tree diversity gradient in the Hainich National Park. Values for beech are set in parentheses. ${ }^{\mathrm{H}}$ host plant specialisation (food or habitat resource), ${ }^{\mathrm{P}}$ prey specialisation.

\begin{tabular}{|c|c|c|c|c|c|}
\hline $\begin{array}{l}\text { Forest } \\
\text { Sstand }\end{array}$ & Strict $^{\mathrm{H}}$ & Intermediate $^{\mathrm{H}}$ & Not Specialised $^{\mathrm{H}}$ & Oligophagous $^{\mathrm{P}}$ & Polyphagous $^{\mathrm{P}}$ \\
\hline 1 & $0(0)$ & $21.4(42.9)$ & $21.4(0)$ & $14.3(28.6)$ & $42.9(28.6)$ \\
\hline 2 & $14.3(9.1)$ & $19(18.2)$ & $28.6(36.4)$ & $19(18.2)$ & $19(27.3)$ \\
\hline $3 a$ & $8.3(0)$ & $25(33.3)$ & $33.3(66.7)$ & $25(33.3)$ & $16.7(0)$ \\
\hline $3 b$ & $0(0)$ & $22.7(18.2)$ & $40.9(45.5)$ & $18.2(36.4)$ & $27.3(18.2)$ \\
\hline 4 & $12.9(0)$ & $16.1(28.6)$ & $32.3(42.9)$ & $29(14.3)$ & $29(42.9)$ \\
\hline 5 & $16(0)$ & $16(20)$ & $32(30)$ & $32(20)$ & $24(20)$ \\
\hline 6 & $13.8(11.1)$ & $17.2(11.1)$ & $27.6(38.9)$ & $24.1(22.2)$ & $20.7(16.7)$ \\
\hline 7 & $15.4(0)$ & $19.2(11.1)$ & $34.6(55.6)$ & $26.9(22.2)$ & $26.9(22.2)$ \\
\hline 8 & $18.2(20)$ & $18.2(20)$ & $27.3(10)$ & $33.3(40)$ & $21.2(20)$ \\
\hline 9 & $17.1(5.3)$ & $24.4(15.8)$ & $29.3(31.6)$ & $24.4(15.8)$ & $34.1(31.6)$ \\
\hline 10 & $22.2(18.8)$ & $22.2(25)$ & $27.8(25)$ & $25(31.3)$ & $27.8(25)$ \\
\hline 11 & $20(21.4)$ & $22.9(14.3)$ & $34.3(35.7)$ & $28.6(35.7)$ & $25.7(14.3)$ \\
\hline
\end{tabular}




\section{CHAPTER}

\section{4}

\section{Canopy vs. understory: How does tree diversity affect bee and wasp communities and their natural enemies across forest strata?}

S. Sobek, T. Tscharntke, C. Scherber, S. Schiele, I. Steffan-Dewenter

(submitted) 


\begin{abstract}
Bees and wasps provide important ecosystem services such as pollination and biocontrol in crop-dominated landscapes, but surprisingly few information is available on hymenopteran communities in temperate forest ecosystems. Species richness and abundance of bees and wasps can by hypothesised to increase with plant diversity, structural complexity, and availability of food and nesting resources. By experimentally exposing standardised nestingsites, we examined abundance and species richness of cavity-nesting bees (pollinators), wasps (predators) and their associated parasitoids across a tree diversity gradient in a temperate deciduous forest habitat. In addition, spatial distribution of individuals and species across forest strata (canopy vs. understory) was tested. Abundance and species richness was high for predatory wasps, but generally low for pollinators. Species-rich forest stands supported increased abundance, but not species richness, of pollinators and predatory wasps, and also increased abundance and species richness of natural enemies. In addition, the forests showed a distinct spatial stratification in that abundance of bees, wasps and parasitoids as well as parasitism rates were higher in the canopy than understory. We conclude that particularly the canopy in temperate forest stands can serve as an important habitat for predatory wasp species and natural enemies, but not bee pollinators. Enhanced tree diversity was related to increased hymenopteran abundance, which is likely to be linked to an increase in nesting and food resources in the mixed forest stands.
\end{abstract}

Key words: beech, ecosystem functioning, Eumeninae, habitat heterogeneity, Hymenoptera, insects, solitary bees and wasps, Sphecidae, trap nests, parasitism, parasitoids, bottom-up control 


\section{Introduction}

In tropical and temperate ecosystems alike, the forest canopy is known as a major source of insect diversity (Erwin 1982, Erwin 1988, Stork 1988, Novotny \& Basset 2005, Dial et al. 2006), and various authors have revealed differences in insect diversity and community composition across forest strata, regardless of the studied taxon (e.g. Le Corff \& Marquis 1999, Schulze et al. 2001, Fermon et al. 2005, Roisin et al. 2006). In a few cases no distinct stratification was observed (Stork \& Grimbacher 2006), and the relative importance of canopy and understory communities to overall diversity patterns and processes may be linked to tree diversity.

Cavity-nesting bees and wasps have been shown to be a particularly useful model system for studying abundance and diversity using hymenopteran pollinators and predators (Tscharntke et al. 1998). Cavity-nesting communities comprise guilds such as pollinators (solitary bees), predators (solitary wasps) and associated parasitoids. They can be easily assessed experimentally by introducing above-ground nesting sites to quantify biodiversity patterns and trophic interactions. Linking biodiversity of a functional group such as parasitoids with ecosystem functioning (i.e. parasitism) is a topical issue in ecology and has recently gained major attention (e.g. Kremen 2005, Hooper et al. 2005, Bianchi et al. 2006, Priess et al. 2007, Klein et al. 2008, Winfree et al. 2008).

Several studies have dealt with the abundance and diversity of cavity-nesting bees and wasps in temperate agricultural (e.g., Gathmann et al. 1994, Kruess \& Tscharntke 2002, Sheffield et al. 2008, Steffan-Dewenter \& Schiele 2008) or tropical agroforesty habitats (e.g. Klein et al. 2006, Tylianakis et al. 2006). In contrast, data on cavity-nesting hymenopteran communities in temperate forest habitats are lacking (but see Taki et al. 2008a). This is remarkable, especially since the proximity to forest habitats has recently been shown to increase local abundance and diversity of cavity-nesting wasps (Holzschuh et al., in press) and also bee pollinators (Taki et al. 2007) in agricultural habitats. Forests are hypothesised to provide resources such as deadwood nesting sites, which are otherwise not present in primarily cleared or simple landscapes. Hence, forest habitats are believed to house source populations of hymenopteran predators, which might spill-over into adjacent agricultural habitats, eventually enhancing biocontrol (Tscharntke et al. 2005, Holzschuh et al., in press).

Taki et al.(2008a, 2008b) provide valuable information on community structure in the forest understory, but it remains ambiguous how cavity nesting bees, wasps and their natural enemies are spatially distributed across forest types and strata. Up to now, virtually nothing is 
known about how the temperate forest canopy contributes to hymenopteran diversity. Here, we present the first data on cavity-nesting hymenopterans and their natural enemies in the canopy and understory of a temperate forest habitat. The study was conducted in the Hainich National Park, Germany's largest coherent deciduous forest. We utilised a semi-natural, mature forest neighbourhood to a priori establish a tree diversity gradient ranging from simple beech to mixed forest stands comprising various deciduous tree species. This allowed us to analyze differences in stratification, and to directly relate our results to tree diversity of the sampled forest stands. Increased plant diversity has been linked to increases in species richness of various arthropod orders (Siemann et al. 1998) including cavity nesting bees and wasps (Tscharntke et al. 1998), and might also influence trophic interactions such as parasitism. We hypothesise that abundance, species richness and parasitism rates of cavitynesting bee and wasp communities increase with increased tree diversity due to enhanced resource availability (e.g. nesting resources, accessory food for parasitoids), and differ between forest strata (canopy vs. understory).

Specifically, we address the following questions: (1) How abundant and species-rich are bee and wasp communities (hereafter referred to as hosts) and their associated parasitoids, and how are they distributed across the tree diversity gradient? (2) How are hosts and parasitoids spatially distributed across forest strata? (3) Do parasitism rates relate to the tree diversity gradient? (4) Do parasitism rates differ between forest strata?

\section{Materials and Methods}

\section{Study area and field sites}

Sampling was conducted in the Hainich National Park, Thuringia, which is Germany's largest coherent semi-natural broadleaved forest. Established in 1997, 7,600 ha of forest (of a total area of 16,000 ha) are currently protected by federal law (Nationalpark Hainich; http://www.nationalpark-hainich.de). To gain a late successional stage of deciduous forest, conifer removal is the only management practice conducted in the park. Sampled forest stands are placed in the north-eastern part of the protected zone south of the village Weberstedt (approximately $51^{\circ} 1^{\prime} \mathrm{N}, 10^{\circ} 5^{\prime} \mathrm{E}$ ).

The mild climate of the research area favours a high variety of deciduous tree species. The examined forest stands were dominated by beech (Fagus sylvatica L.), lime (Tilia platyphyllos Scop., Tilia cordata L.) and ash (Fraxinus excelsior L.). The study site has an elevation of $350 \mathrm{~m}$ a. s. 1. and a temperate climate, with an average temperature of $+7.5{ }^{\circ} \mathrm{C}$ (1973-2004, Deutscher Wetterdienst). Average annual temperature in 2006 was $9.4{ }^{\circ} \mathrm{C}$ 
(Meteomedia 2006). Mean annual precipitation is $590 \mathrm{~mm}$ (1973-2004, Deutscher Wetterdienst), $518 \mathrm{~mm}$ in 2006 (Meteomedia 2006). The predominant soil type is stagnic luvisol on loess-limestone as parent material.

Trap nests were installed in 12 forest stands of different tree diversity, which were selected $a$ priori to test for effects of a naturally occurring tree diversity gradient on arthropod communities and other biotic and abiotic variables (see Leuschner et al., in press). All forest stands had remained undisturbed for more than 40 years since the last logging event, and shared major characteristics like soil type, flat elevation, and absence of canopy gaps. In each stand a $50 \times 50 \mathrm{~m}$ plot was fenced for determination of tree diversity. To account for the relative abundance of trees as well as for species richness, tree diversity of the plots was described as Shannon index based on stem counts of trees with a dbh (diameter breast height) $>7 \mathrm{~cm}$ (Magurran 2004). The selected forest stands represented a gradient ranging from simple beech to mixed forests with up to 11 deciduous tree species. (i.e. Acer platanoides L., Acer pseudoplatanus L., Carpinus betulus L., Fagus sylvatica L., Fraxinus excelsior L., Prunus avium (L.), Quercus robur L., Sorbus torminalis L., Tilia cordata L., Tilia platyphyllos L., Ulmus glabra Huds.).

\section{Trap nest sampling and sample processing}

Sampling of bees and wasps was accomplished using trap nests. Trap nests have been utilised in several studies in agricultural and tropical forest ecosystems to assess diversity of aboveground cavity nesting bees and wasps and their natural enemies (e. g. Tscharntke et al. 1998; Klein et al. 2002). The trap nests consisted of two paired PVC tubes (each ø $10.5 \mathrm{~cm}$ ) filled with a random mix of reed internodes (Phragmites australis (Cav). Trin. ex Steud.) ranging from $0.2 \mathrm{~cm}$ to approximately $1 \mathrm{~cm}$ in diameter. In contrast to random trapping with flight interception or Malaise traps, capture of tourist species can be eliminated by experimentally offering nesting resources which are only colonised by species capable to reproduce in a certain area (Tscharntke et al. 1998). Furthermore, cavity nesting species tend to breed in close proximity to the nesting site they originated from. Hence, experimental exposure of these standardised nesting resources are particularly useful to describe communities of bees, wasps and their parasitoids within a defined habitat.

Trap nests were mounted in the canopy and understory of all forest stands. In total, 144 trap nests (12 trap nests per stand; 6 canopy trap nests, 6 understory trap nests) were installed in the centre of individual tree crowns using a crossbow, or mounted at breast height on wooden posts in the understory next to the sampled tree. The sampling scheme was based on the 
relative abundance of beech. In highly beech dominated stands with up to four tree species (83-100\% beech), trap nests were mounted in beech (Fagus sylvatica), in stands with up to seven tree species and at least $48 \%$ beech, trap nests were installed in the three most dominant species (beech, lime (Tilia sp.), ash (Fraxinus excelsior), and in stands with up to 11 tree species and the proportion of beech decreasing below $42 \%$, trap nests were installed in six tree species (beech, lime, ash, sycamore maple (Acer pseudoplatanus), hornbeam (Carpinus betulus) and either oak (Quercus robur) or service tree (Sorbus torminalis)). Sampled trees were randomly selected within the $50 \times 50 \mathrm{~m}$ plots or in the directly adjacent forest.To quantify a possible nesting resource for hymenopteran hosts, the amount of deadwood $\left[\mathrm{m}^{3} / \mathrm{m}^{2}\right]$ was measured within four circular subplots with a radius of $5 \mathrm{~m}$.

The trap nests were exposed over a period of six months starting in early May 2006, and harvested in late September of the same year. After retrieval, the traps were stored at $4^{\circ} \mathrm{C}$ to initiate diapause. After a period of five months, the traps were inspected visually for internodes occupied with nests. Internodes with nests were dissected and the number of host brood cells, the number of brood cells infested with parasitoids and number of parasitoid eggs, as well as host and parasitoid morphospecies was recorded. The nests were then placed in individual glass vials and stored at room temperature for rearing. Upon emergence, all individuals were pinned and mounted for identification. Voucher specimens were deposited in an in-house collection (Agroecology, Georg-August-University Göttingen). When the condition of the material did not allow for species-level identification, specimens were assigned to other taxonomic levels (at least to family). Ichneumonid wasps were grouped into morphospecies.

\section{Data analyses}

Total species richness was calculated separately for each trap nesting guild (hosts and parasitoids) as accumulated number of species per plot and stratum (pooled across samples). Because of the low abundance and species richness of host bees (only three species present), we pooled bees and wasps in all further analyses.

Using the lmer function in $\mathrm{R}$ (package lme4), we fitted generalised linear mixed effects models (GLMMs) to account for poisson error distribution (response variables: abundance and species richness of hosts/parasitoids) and binomial error distribution (response variable: parasitism). A maximal model was fitted with the following sequence: fixed effects = deadwood $\mathrm{m}^{3} / \mathrm{m}^{2}$, Shannon index (tree diversity), stratum (canopy/understory), Shannon index $\mathrm{x}$ stratum. Random effects were included as differences between intercepts (i.e., different 
intercepts either for plots or for stratum nested within plots). The amount of deadwood $\left[\mathrm{m}^{3} / \mathrm{m}^{2}\right]$ was included for hosts only to quantify availability of a possible nesting resource. Model residuals were examined for meeting assumptions of normality and homoscedasticity. Similarity of cavity-nesting host communities in different strata of different forest stands was determined using the Bray-Curtis similarity index. The data were transformed into a matrix of dissimilarity (1 minus Bray-Curtis; Zuur et al. 2007) and used as input data for a metric multidimensional scaling of the different communities.

Bray-Curtis similarity indices were computed with EstimateS 8.0 (Colwell 2006). Other statistical analyses were carried out using R, Version 2.6.2 (R Development Core Team 2007; http://www.R-project.org).

\section{Results}

\section{General community structure}

In total, the trap nests were occupied with 3,948 host brood cells. The majority of brood cells was built by five species of eumenid wasps (Hymenoptera: Eumeninae, 1776 cells (45.1\%), Appendix 4.1), followed by seven species of sphecid wasps (Hymenoptera: Sphecidae, 1,272 cells $(32.2 \%)$ ) and one spider wasp species (Hymenoptera: Pompilidae, 548 cells (13.9\%)). Solitary cavity-nesting bees were less species-rich (three species) than wasps and showed the lowest abundance of only 347 (8.8\%) brood cells. A total of 566 brood cells was occupied by 14 species of natural enemies, the majority thereof hymenopteran parasitoids (12 species in seven families, Appendix 4.2).

\section{Stratification of cavity-nesting communities and parasitism}

Hosts were significantly more abundant in the canopy $\left(216.2 \pm 24.5, \chi^{2}=396.13, d f=4, \mathrm{p}<\right.$ 0.001 , Table 4.1) compared to the forest understory $(112.8 \pm 8.9)$, and abundance in the canopy increased significantly across the tree diversity gradient $\left(\chi^{2}=7.13, d f=3, \mathrm{p}=0.008\right.$, Fig. 4.1a). Canopy as well as understory communities did not respond significantly to deadwood availability, neither by means of abundance nor by species richness, and species richness was also not related to tree diversity $\left(\chi^{2}=3.01, d f=3, p=0.083\right.$, Fig. 4.1c). 


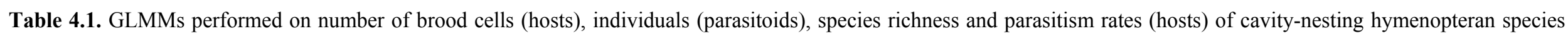

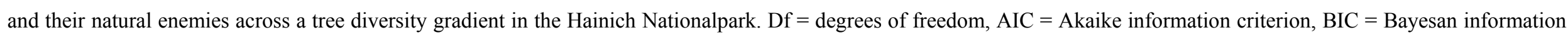
criterion, $\log \mathrm{Lik}=\log$ likelihood.

\begin{tabular}{|c|c|c|c|c|c|c|c|}
\hline \multicolumn{8}{|l|}{ Response Variable: Host Brood Cells } \\
\hline Models: & Df & AIC & BIC & logLik & $\chi^{2}$ & $\mathbf{p}$ & Explanatory Variable \\
\hline lme1d: Brood Cells $\sim(1 \mid$ Plot $)$ & 2 & 668.74 & 671.1 & 332.37 & & & \\
\hline lme1c: Brood Cells $\sim$ Shannon $+(1 \mid$ Plot $)$ & 3 & 663.61 & 667.15 & 328.81 & 7.13 & 0.008 & Shannon (Tree Diversity) \\
\hline lme1b: Brood Cells $\sim$ Shannon + Stratum $+(1 \mid$ Plot $)$ & 4 & 269.48 & 274.19 & 130.74 & 396.13 & $<\mathbf{0 . 0 0 1}$ & Stratum \\
\hline lme1a: Brood Cells $\sim$ Shannon $*$ Stratum $+(1 \mid$ Plot $)$ & 5 & 222.69 & 228.58 & 106.35 & 48.79 & $<\mathbf{0 . 0 0 1}$ & Stratum x Shannon \\
\hline lme1: Brood Cells $\sim$ DeadWood + Shannon $*$ Stratum $+(1 \mid$ Plot $)$ & 6 & 223.33 & 230.4 & 105.66 & 1.36 & 0.243 & Deadwood \\
\hline
\end{tabular}

Response Variable: Host Species

Models:

lme2d: Host Species $\sim(1 \mid$ Plot $)$

lme2c: Host Species $\sim$ Shannon $+(1 \mid$ Plot $)$

lme2b: Host Species $\sim$ Shannon + Stratum $+(1 \mid$ Plot $)$

lme2a: Host Species $\sim$ Shannon * Stratum $+(1 \mid$ Plot $)$

lme2: Host Species $\sim$ DeadWood + Shannon $*$ Stratum $+(1 \mid$ Plot $)$

$\begin{array}{lllllll}2 & 27.63 & 29.99 & -11.82 & & & \\ 3 & 26.62 & 30.15 & -10.31 & 3.01 & 0.083 & \text { Shannon (Tree Diversity) } \\ 4 & 22.41 & 27.12 & -7.21 & 6.21 & \mathbf{0 . 0 1 3} & \text { Stratum } \\ 5 & 23 & 28.89 & -6.5 & 1.41 & 0.235 & \text { Stratum x Shannon } \\ 6 & 24.66 & 31.72 & -6.33 & 0.34 & 0.559 & \text { Deadwood }\end{array}$

Response Variable: Parasitoid Individuals

Models:

lme3c: Parasitoid Individuals $\sim(1 \mid$ Plot $)$

$2 \quad 174.61 \quad 176.96 \quad-85.3$

lme3b: Parasitoid Individuals $\sim$ Shannon $+(1 \mid$ Plot $)$

$\begin{array}{lllll}3 & 170.2 & 173.73 & -82.1 & 6.4\end{array}$

lme3a: Parasitoid Individuals $\sim$ Shannon + Stratum $+(1 \mid$ Plot $)$

$\begin{array}{lllll}4 & 73.24 & 77.95 & -32.61 & 98.96\end{array}$

0.011 Shannon (Tree Diversity)

$<\mathbf{0 . 0 0 1}$ Stratum 
Response Variable: Parasitoid Species

\section{Models}

lme4c: Parasitoid Species $\sim(1 \mid$ Plot $)$

lme4b: Parasitoid Species $\sim$ Shannon $+(1 \mid$ Plot $)$

lme4a: Parasitoid Species $\sim$ Shannon + Stratum $+(1 \mid$ Plot $)$

lme4: Parasitoid Species $\sim$ Shannon $*$ Stratum $+(1 \mid$ Plot $)$

$\begin{array}{lllllll}2 & 23.53 & 25.89 & -9.77 & & & \\ 3 & 20.17 & 23.7 & -7.08 & 5.37 & \mathbf{0 . 0 2 1} & \text { Shannon (Tree Diversity) } \\ 4 & 16.29 & 21 & -4.1 & 5.88 & \mathbf{0 . 0 1 5} & \text { Stratum } \\ 5 & 18.09 & 23.98 & -4.05 & 0.2 & 0.656 & \text { Stratum x Shannon }\end{array}$

\section{Response Variable: Parasitism}

Models

lme5c: Parasitism $\sim(1 \mid$ Plot $)$

lme5b: Parasitism $\sim$ Shannon $+(1 \mid$ Plot $)$

lme5a: Parasitism $\sim$ Shannon + Stratum $+(1 \mid$ Plot $)$

$2 \quad 57.78 \quad 60.14 \quad-26.89$

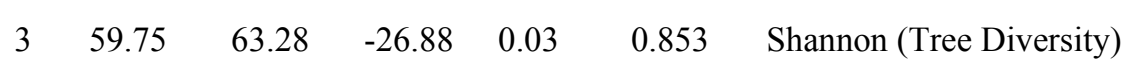

lme5: Parasitism $\sim$ Shannon $*$ Stratum $+(1 \mid$ Plot $)$

$\begin{array}{lllllll}4 & 54.93 & 59.64 & -23.47 & 6.82 & \mathbf{0 . 0 0 9} & \text { Stratum }\end{array}$

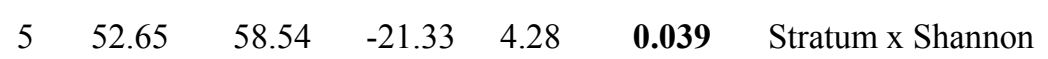




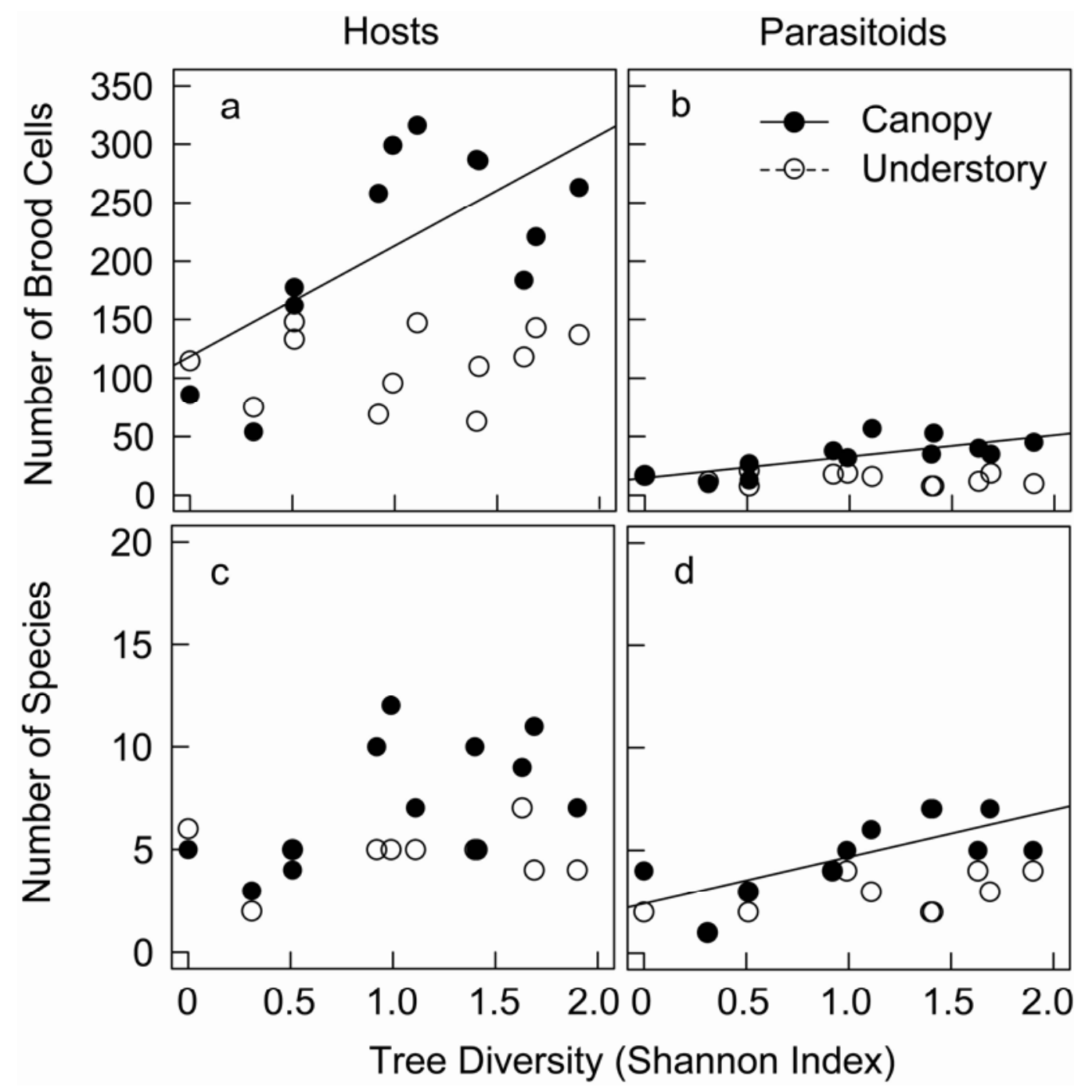

Fig. 4.1. Stratification of cavity-nesting bees and wasps and their natural enemies across a tree diversity gradient. a and b) Number of brood cells or parasitoid individuals in the canopy and understory, b and c) species richness in the canopy and understory. (a, c hosts, b, d parasitoids).

Parasitoids were more abundant in the canopy $(33.5 \pm 4.3)$ compared to the understory $(14 \pm$ $1.4 ; \chi^{2}=98.96, d f=4, \mathrm{p}<0.001$, Fig. 4.1b), and abundance as well as species richness in the canopy increased significantly with increased tree species richness (parasitoid abundance: $\chi^{2}=$ 6.4, $d f=3, \mathrm{p}=0.011$, Fig. 4.1b, parasitoid species richness: $\chi^{2}=5.37, d f=3, \mathrm{p}=0.021$, Fig. 4.1d). More species of parasitoids were observed in the canopy $(4.8 \pm 0.5)$ compared to the understory $\left(2.8 \pm 0.3, \chi^{2}=5.8, d f=4, \mathrm{p}=0.015\right.$, Fig. 4.1d).

Multidimensional scaling of Bray-Curtis similarity illustrated that species composition of hosts differed between the canopy and understory (Fig. 4.2). Parasitism rates also differed between strata (canopy $15.8 \pm 0.9 \%$, understory $13.2 \pm 1.7 \% ; \chi^{2}=6.82, d f=4, \mathrm{p}=0.009$, Table 4.1), but did not respond to the tree diversity gradient (Fig. 4.3). 


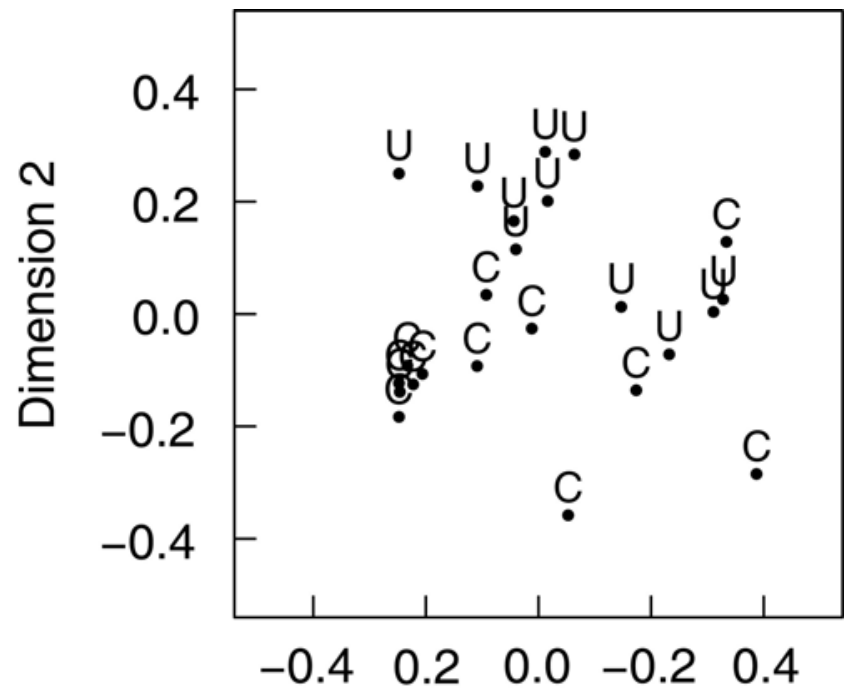

Dimension 1

Fig. 4.2. Metric multidimensional scaling (based on Bray-Curtis-similarity) of cavity-nesting bee and wasp host communities across a tree diversity gradient. $\mathrm{C}=$ canopy, $\mathrm{U}=$ understory.

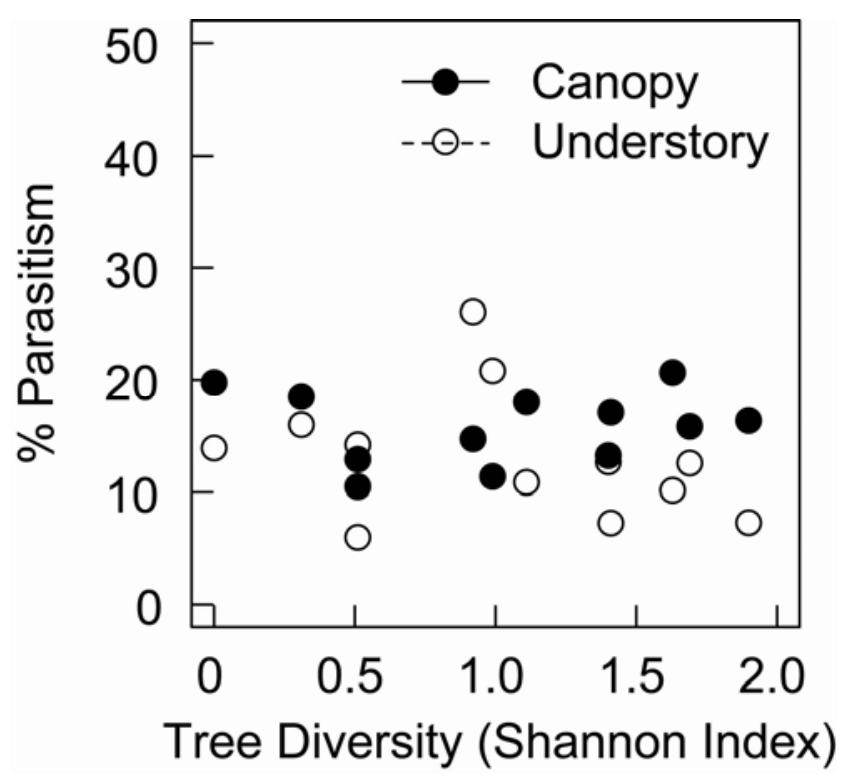

Fig. 4.3. Proportion of hymenopteran brood cells parasitised by natural enemies in the canopy and understory across a tree diversity gradient in a Central European deciduous forest. 


\section{Discussion}

Our results show that increased tree diversity of deciduous forest stands enhanced abundance, but not species richness, of cavity-nesting bees and wasps. In addition, the forests showed a distinct spatial stratification in that abundance of bees, wasps and parasitoids as well as parasitism rates were higher in the canopy than understory. Tree diversity determined various biotic and abiotic characteristics of the sampled study sites, such as species richness and density of understory vegetation which increased across the a priori set tree diversity gradient (Mölder et al. 2006). Highly structured, heterogeneous vegetation, as found in the most diverse forest stands in contrast to the simple beech stands, provides various resources like food and sites for reproduction, colonisation, and overwintering of arthropods (Lawton 1983), which can be expected to support a more abundant, even though not more diverse, cavitynesting community.

The relationships between abundance or species richness of cavity-nesting communities and plant diversity is little known (but see Gathmann et al., 1994, Tscharntke et al. 1998, Sheffield et al. 2008). Although tree species richness, and with it increased habitat heterogeneity and resource availability, has been shown to increase species richness of arthropods, for example in ants and parasitic wasps (e.g. Ribas et al. 2003, Sperber et al. 2004), cavity-nesting taxa may respond differently. The increased abundance of hosts in diverse forest stands as observed in our study might be a result of a greater availability of nesting sites and food resources. Surprisingly, the deadwood amount within stands had no effect on abundance or species richness of cavity-nesting host species. This indicates that rather than deadwood as such, a higher diversity of prey items or the quality of actual deadwood nesting sites might have been of importance. Cavity nesting species utilize abandoned galleries of saproxylic beetles for nesting, and especially deadwood suspended in the canopy might serve as an important nesting-site resource as opposed to deadwood on the forest floor. Beetle abundance in the canopy increased across the tree diversity gradient (Sobek et al., submitted), and prey such as caterpillars and spiders may follow a similar pattern.

Interestingly, the number of host bee species observed in this study was considerably low compared to studies conducted in agricultural or grassland habitats (excluding parasitic bees, three species vs. at least 13 species (e. g. Steffan-Dewenter 2003, Steffan-Dewenter \& Leschke 2003, Albrecht et al. 2007), and even lower than in North American temperate forests (no bees, Taki et al. 2008b). Rather than on high floral diversity as such, bees often 
depend more on the density of floral resources (Höhn et al. submitted a). Even though understory plant cover and species richness increased across the tree diversity gradient (Mölder et al. 2006), the mere number of available flowering resources might have simply not been enough to sustain a diverse and abundant community. In the most diverse forest stands of our study, understory vegetation was generally dense and dominated by non-flowering plants like tree saplings, which overgrew available flowers resulting in reduced accessibility for pollinators. Hence, limited availability and accessibility of pollen and nectar resources appeared to make forest habitats rather unsuitable for cavity-nesting bees, even though the availability of nesting-sites might be enhanced compared to other habitat types.

Parasitoid abundance and species richness was related to tree diversity and showed a distinct stratification between canopy and understory. Even though higher trophic levels do not directly depend on the same resources as their hosts (nesting sites, floral resources), they are indirectly connected to these forest stand resources by availability of suitable hosts, in terms of numbers and species. In our study, the majority of parasitoids was linked to only one or two host species (Appendix 2). Parasitism rates differed between forest strata and were slightly higher in the canopy compared to the understory. A stratification of parasitism rates could not be shown for a solitary cavity-nesting wasp species (Eumeninae) in a tropical forest habitat (Höhn et al., submitted b), and might in our case be related to a slightly more diverse parasitoid community in the canopy.

In conclusion, species-rich forest stands showed increased abundance of cavity-nesting hymenopterans, which might enhance provision of ecosystem services like pollination and biocontrol of herbivores (e.g. aphids, lepidopteran caterpillars), even though species richness was not increased. Furthermore, temperate forest stands show a distinct stratification of hymenopteran abundance and species composition, most likely related to a greater availability of nesting-sites and food resources in the forest canopy. Hence we conclude that tree diversity in temperate forests is a predictor of insect distribution patterns and associated processes such as parasitism. 


\section{Acknowledgements}

This study gained financial support by the DFG [German Research Foundation] within the Research Training Group "Graduiertenkolleg 1086: The role of biodiversity for biogeochemical cycles and biotic interactions in temperate deciduous forests“. We highly acknowledge Christoph Leuschner, Frank Thomas, Hermann Jungkunst and Stefan Fleck for group coordination and also thank Mascha Jacob, Karl M. Daenner and Mareen Gollnow for providing data on stand characteristics. Our special thanks go to Andreas Dittrich, Martin Erren, Tobias Gebauer, Martin Goßner, Friedrich Rathing and Ulrich Simon for assistance with field work.

\section{References}

Albrecht, M., Duelli, P., Schmid, B., \& Müller, C.B. (2007) Interaction Diversity Within Quantified Insect Food Webs in Restored and Adjacent Intensively Managed Meadows. Journal of Animal Ecology 76, 1015-1025.

Bianchi, F., Booij, C.J.H., \& Tscharntke, T. (2006) Sustainable Pest Regulation in Agricultural Landscapes: a Review on Landscape Composition, Biodiversity and Natural Pest Control. Proceedings of the Royal Society B-Biological Sciences 273, 1715-1727.

Colwell, R.K. (2006) EstimateS: Statistical Estimation of Species Richness and Shared Species from Samples. Version 7.5. User's guide and application published at http://purl.oclc.org/estimates.

Dial, R.J., Ellwood, M.D.F., Turner, E.C., \& Foster, W.A. (2006) Arthropod Abundance, Canopy Structure, and Microclimate in a Bornean Lowland Tropical Rain Forest. Biotropica 38, 643-652.

Erwin, T.L. (1982) Tropical Forests: Their Richness in Coleoptera and Other Arthropod Species. The Coleopterist's Bulletin 36, 74-75.

Erwin, T.L. (1988) The Tropical Forest Canopy- The Heart of Biotic Diversity. In: Wilson, E.O. (Ed.)., Biodiversity. National Academy Press.

Fermon, H., Waltert, M., Vane-Wright, R.I., \& Mühlenberg, M. (2005) Forest Use and 
Vertical Stratification in Fruit-Feeding Butterflies of Sulawesi, Indonesia: Impacts for Conservation. Biodiversity and Conservation 14, 333-350.

Gathmann, A., Greiler, H.J., \& Tscharntke, T. (1994) Trap-Nesting Bees and Wasps Colonising Set-Aside Fields - Succession and Body-Size, Management by Cutting and Sowing. Oecologia 98, 8-14.

Höhn, P., Steffan-Dewenter, I., Lewis, O.T., \& Tscharntke, T. (submitted a) Relative Contribution of Cacao Agroforestry, Rainforest and Openland to Local and Regional Bee Diversity.

Höhn, P., Steffan-Dewenter, I., \& Tscharntke, T., (submitted b) Rainforest Conversion and Agroforestry Intensification Reverse Vertical Distribution of Hymenoptera Diversity.

Holzschuh, A., Steffan-Dewenter, I., \& Tscharntke, T. (in press) Grass strip corridors in agricultural landscapes enhance nest site colonisation by solitary wasps. Ecological Applications.

Hooper, D.U., Chapin, F.S., Ewel, J.J., Hector, A., Inchausti, P., Lavorel, S., Lawton, J.H., Lodge, D.M., Loreau, M., Naeem, S., Schmid, B., Setala, H., Symstad, A.J., Vandermeer, J., \& Wardle, D.A. (2005) Effects of Biodiversity on Ecosystem Functioning: a Consensus of Current Knowledge. Ecological Monographs 75, 3-35.

Klein, A.M., Cunningham, S.A., Bos, M., \& Steffan-Dewenter, I. (2008) Advances in Pollination Ecology From Tropical Plantation Crops. Ecology 89, 935-943.

Klein, A.M., Steffan-Dewenter, I., Buchori, D., \& Tscharntke, T. (2002) Effects of Land-Use Intensity in Tropical Agroforestry Systems on Coffee Flower-Visiting and Trap-Nesting Bees and Wasps. Conservation Biology 16, 1003-1014.

Klein, A.M., Steffan-Dewenter, I., \& Tscharntke, T. (2006) Rain Forest Promotes Trophic Interactions and Diversity of Trap-Nesting Hymenoptera in Adjacent Agroforestry. Journal of Animal Ecology 75, 315-323.

Kremen, C., 2005. Managing Ecosystem Services: What Do We Need to Know About Their Ecology? Ecology Letters 8, 468-479.

Kruess, A. \& Tscharntke, T. (2002) Grazing Intensity and the Diversity of Grasshoppers, Butterflies, and Trap-Nesting Bees and Wasps. Conservation Biology 16, 1570-1580. 
Lawton, J.H. (1983) Plant Architecture and the Diversity of Phytophagous Insects. Annual Review of Entomology 28, 23-29.

Le Corff, J. \& Marquis, R.J. (1999) Differences Between Understorey and Canopy in Herbivore Community Composition and Leaf Quality for Two Oak Species in Missouri. Ecological Entomology 24, 46-58.

Leuschner, C., Jungkunst, H.F. \& Fleck, S. (in press) Studying the Functional Role of Tree Diversity in Forests: the Pros and Cons of Synthetic Stands and Across-Site Comparisons in Established Forests. Basic and Applied Ecology.

Magurran, A.E. (2004) Measuring Biological Diversity. Blackwell, Oxford.

Mölder, A., Bernhardt-Römermann, M., \& Schmidt, W. (2006) Forest Ecosystem Research in Hainich National Park (Thuringia): First Results on Flora and Vegetation in Stands with Contrasting Tree Species Diversity. Waldökologie online 3, 83-99.

Novotny, V. \& Basset, Y. (2005) Review - Host Specificity of Insect Herbivores in Tropical Forests. Proceedings of the Royal Society B-Biological Sciences 272, 1083-1090.

Pinheiro, J.C. \& Bates, D.M. (2000) Mixed-Effects Models in S and S-PLUS. Springer, New York, London.

Priess, J.A., Mimler, M., Klein, A.M., Schwarze, S., Tscharntke, T., \& Steffan-Dewenter, I. (2007) Linking Deforestation Scenarios to Pollination Services and Economic Returns in Coffee Agroforestry Systems. Ecological Applications 17, 407-417.

Ribas, C.R., Schoereder, J.H., Pic, M., \& Soares, S.M. (2003) Tree Heterogeneity, Resource Availability, and Larger Scale Processes Regulating Arboreal Ant Species Richness. Austral Ecology 28, 305-314.

Roisin, Y., Dejean, A., Corbara, B., Orivel, J., Samaniego, M., \& Leponce, M. (2006) Vertical Stratification of the Termite Assemblage in a Neotropical Rainforest. Oecologia 149, 301311.

Schulze, C.H., Linsenmair, K.E., \& Fiedler, K. (2001) Understorey Versus Canopy: Patterns of Vertical Stratification and Diversity Among Lepidoptera in a Bornean Rain Forest. Plant Ecology 153, 133-152. 
Sheffield, C.S., Kevan, P.G., Westby, S.M., \& Smith, R.F. (2008) Diversity of Cavity-Nesting Bees (Hymenoptera : Apoidea) Within Apple Orchards and Wild Habitats in the Annapolis Valley, Nova Scotia, Canada. Canadian Entomologist 140, 235-249.

Siemann, E., Tilman, D., Haarstad, J., \& Ritchie, M. (1998) Experimental Tests of the Dependence of Arthropod Diversity on Plant Diversity. The American Naturalist 152, 738750 .

Sobek, S., Scherber, C., Steffan-Dewenter, I., \& Tscharntke, T. (submitted) Spatiotemporal Changes of Beetle Communities Across a Tree Diversity Gradient.

Sperber, C.F., Nakayama, K., Valverde, M.J., \& Neves, F.D. (2004) Tree Species Richness and Density Affect Parasitoid Diversity in Cacao Agroforestry. Basic and Applied Ecology 5, 241-251.

Steffan-Dewenter, I. (2003) Importance of Habitat Area and Landscape Context for Species Richness of Bees and Wasps in Fragmented Orchard Meadows. Conservation Biology 17, 1036-1044.

Steffan-Dewenter, I. \& Leschke, K. (2003) Effects of Habitat Management on Vegetation and Above-Ground Nesting Bees and Wasps of Orchard Meadows in Central Europe. Biodiversity and Conservation 12, 1953-1968.

Steffan-Dewenter, I. \& Schiele, S. (2008) Do Resources or Natural Enemies Drive Bee Population Dynamics in Fragmented Habitats? Ecology 89, 1375-1387.

Stork, N.E. (1988) Insect Diversity: Facts, Fiction and Speculation. Biological Journal of the Linnean Society 35, 321-337.

Stork, N.E. \& Grimbacher, P.S. (2006) Beetle Assemblages From an Australian Tropical Rainforest Show That the Canopy and the Ground Strata Contribute Equally to Biodiversity. Proceedings of the Royal Society B-Biological Sciences 273, 1969-1975.

Taki, H., Kevan, P.G., \& Ascher, J.S. (2007) Landscape Effects of Forest Loss in a Pollination System. Landscape Ecology 22, 1575-1587.

Taki, H., Kevan, P.G., Viana, B.F., Silva, F.O., \& Buck, M. (2008a) Artificial Covering on Trap Nests Improves the Colonisation of Trap-Nesting Wasps. Journal of Applied Entomology 132, 225-229. 
Taki, H., Viana, B.F., Kevan, P.G., Silva, F.O., \& Buck, M. (2008b) Does Forest Loss Affect the Communities of Trap-Nesting Wasps (Hymenoptera : Aculeata) in Forests? Landscape Vs. Local Habitat Conditions. Journal of Insect Conservation 12, 15-21.

Tscharntke, T., Gathmann, A., \& Steffan-Dewenter, I. (1998) Bioindication Using TrapNesting Bees and Wasps and Their Natural Enemies: Community Structure and Interactions. Journal of Applied Ecology 35, 708-719.

Tscharntke, T., Rand, T.A., \& Bianchi F. (2005) The Landscape Context of Trophic Interactions: Insect Spillover Across the Crop-Noncrop Interface. Annales Zoologici Fennici 42, 421-432.

Tylianakis, J.M., Klein, A.M., Lozada, T., \& Tscharntke, T. (2006) Spatial Scale of Observation Affects Alpha, Beta and Gamma Diversity of Cavity-Nesting Bees and Wasps Across a Tropical Land-Use Gradient. Journal of Biogeography 33, 1295-1304.

Winfree, R., Williams, N.M., Gaines, H., Ascher, J.S., \& Kremen, C. (2008) Wild Bee Pollinators Provide the Majority of Crop Visitation Across Land-Use Gradients in New Jersey and Pennsylvania, USA. Journal of Applied Ecology 45, 793-802.

Zuur, A.F., Ieno, E.N., \& Smith G. M. (2007) Analysing Ecological Data. Springer, Heidelberg. 


\section{Appendix 4.1}

List of cavity-nesting bee and wasp species collected in the canopy and understory of forest stands across a tree diversity gradient in the Hainich National Park. $\mathrm{n}^{\mathrm{C}}=$ number of individuals captured in the canopy, $\mathrm{n}^{\mathrm{U}}=$ number of individuals captured in the understory, $\mathrm{n}^{\mathrm{P}}=$ number of observed parasitoid species.

\begin{tabular}{lllll}
\hline Family/Subfamily & Species & $\mathrm{n}^{\mathrm{C}}$ & $\mathrm{n}^{\mathrm{U}}$ & $\mathrm{n}^{\mathrm{P}}$ \\
\hline Colletidae & Hylaeus communis & 295 & 32 & 2 \\
Colletidae & Hylaeus confusus & 0 & 9 & 0 \\
Megachilidae & Megachile ligniseca & 11 & 0 & 1 \\
Eumeninae & Ancistrocerus antilope & 9 & 0 & 1 \\
Eumeninae & Ancistrocerus trifasciatus & 985 & 599 & 8 \\
Eumeninae & Discoelius zonalis & 162 & 0 & 2 \\
Eumeninae & Symmorphus debilitatus & 6 & 0 & 0 \\
Eumeninae & Symmorphus gracilis & 10 & 10 & 0 \\
Pompilidae & Dipogon subintermedius & 271 & 277 & 4 \\
Sphecidae & Crossocerus binotatus & 11 & 5 & 2 \\
Sphecidae & Nitela spinolae & 38 & 7 & 1 \\
Sphecidae & Passaloecus corniger & 54 & 5 & 3 \\
Sphecidae & Passaloecus insignis & 64 & 4 & 2 \\
Sphecidae & Passaloecus sp. & 1 & 0 & 0 \\
Sphecidae & Psenulus pallipes & 73 & 10 & 3 \\
Sphecidae & Rhopalum clavipes & 42 & 72 & 1 \\
Sphecidae & Trypoxylon clavicerum & 562 & 324 & 8 \\
\hline
\end{tabular}




\section{Appendix 4.2}

List of natural enemies of cavity-nesting hymenopterans collected in the canopy and understory of forest stands across a tree diversity gradient in the Hainich National Park. $\mathrm{n}^{\mathrm{C}}=$ number of individuals captured in the canopy, $\mathrm{n}^{\mathrm{U}}=$ number of individuals captured in the understory, $\mathrm{n}^{\mathrm{H}}=$ number of observed host species.

\begin{tabular}{llll}
\hline Morpho)Species & $\mathrm{n}^{\mathrm{C}}$ & $\mathrm{n}^{\mathrm{U}}$ & $\mathrm{n}^{\mathrm{H}}$ \\
\hline Braconidae 1 & 1 & 0 & 1 \\
Chrysididae & 10 & 5 & 2 \\
Chrysis ignita agg. & 68 & 7 & 1 \\
Coelioxys alata & 3 & 0 & 1 \\
Diptera & 0 & 7 & 1 \\
Gasteruption assectator & 18 & 0 & 1 \\
Ichneumonidae & 24 & 12 & 2 \\
Ichneumonidae 1 & 30 & 2 & 4 \\
Ichneumonidae 2 & 39 & 7 & 4 \\
Ichneumonidae 3 & 2 & 0 & 1 \\
Ichneumonidae 4 & 111 & 86 & 3 \\
Ichneumonidae 5 & 1 & 0 & 1 \\
Ichneumonidae 6 & 0 & 1 & 1 \\
Ichneumonidae 7 & 0 & 1 & 1 \\
Megatoma undata & 52 & 3 & 8 \\
Melittobia acasta & 41 & 36 & 4 \\
Not identified & 2 & 1 & 2 \\
\hline
\end{tabular}




\section{CHAPTER}

\section{5}

Sapling herbivory, herbivores and predators across a semi-natural tree diversity gradient in Germany's largest connected deciduous forest

S. Sobek, C. Scherber, I. Steffan-Dewenter, T. Tscharntke

(submitted) 


\begin{abstract}
Tree species-rich forests are hypothesised to be less susceptible to insect herbivores, but so far herbivory-diversity relationships have rarely been tested for tree saplings, and no such study has been published for deciduous forests in Central Europe. We expected that diverse tree communities reduce the probability of detection of host plants and increase abundance of predators, thereby reducing herbivory. We examined levels of herbivory suffered by beech (Fagus sylvatica L.) and maple saplings (Acer pseudoplatanus L. and Acer platanoides L.) across a tree species diversity gradient within Germany's largest remaining deciduous forest area, and investigated whether simple beech or mixed stands were less prone to damage caused by herbivorous insects. Leaf area loss and the frequency of galls and mines were recorded for 1,040 saplings (>13,000 leaves) in June and August 2006. In addition, relative abundance of predators was assessed to test for potential top-down control. Leaf area loss was generally higher in the two species of maple compared to beech saplings, while only beech showed a decline in damage caused by leaf-chewing herbivores across the tree diversity gradient. No significant patterns were found for galls and mines. Relative abundance of predators on beech showed a seasonal response and increased on species-rich plots in June, suggesting higher biological control. We conclude that in temperate deciduous forests herbivory-tree diversity relationships are significant, but tree species-dependent with bottomup and top-down control as possible mechanisms. In contrast to maple, beech profits from growing in a neighbourhood of higher tree richness, which implies that species identity effects may be of greater importance than tree diversity effects per se. Hence, herbivory on beech appeared to be mediated bottom-up by resource concentration in the sampled forest stands, as well as regulated top-down through biocontrol by natural enemies.
\end{abstract}

Keywords: diversity-functioning relationships, leaf damage, mines, multitrophic interactions, plant-animal interactions. 


\section{Introduction}

The relationship between plant biodiversity and ecosystem functioning is a central question in ecology (Hooper et al. 2005, Balvanera et al. 2006, Cardinale et al. 2006), but so far the majority of studies have focused on plant productivity in experimental grasslands. Research on the effects of plant diversity on other trophic levels, for example insect herbivores as primary consumers, has a long tradition in agricultural habitats (reviewed in Andow 1991), while natural habitat types have only recently begun to be considered. In most of these studies, reduced insect herbivory was observed with increased plant diversity, both in agricultural (Risch et al. 1983) and grassland (Unsicker et al. 2006) or forest habitats (Jactel et al. 2006, Jactel \& Brockerhoff 2007, Kaitaniemi et al. 2007). However, some authors found the opposite (Vehviläinen et al. 2006) or no effect at all (Scherber et al. 2006), and the outcome often appears to be species dependent (Jactel \& Brockerhoff 2007, Vehviläinen et al. 2007). Identity of the observed plant species and of species in the surrounding community, as well as host specificity of herbivores, have been shown to affect the herbivory-plant diversity relationship (Koricheva et al. 2006, Unsicker et al. 2006, Jactel \& Brockerhoff 2007). According to data from other invertebrate herbivores (e.g. molluscs), diversity-herbivory relationships are not controlled by plant diversity in the local neighboorhood, but by plant diversity observed on community level (Hanley 2004).

Lower susceptibility of species-rich plant communities to insect herbivores, also described as associational resistance (Tahvanainen \& Root 1972, Karban 2007, Sholes 2008), can be explained with two well-established concepts: the resource concentration hypothesis (Tahvanainen \& Root 1972, Root 1973) and the enemies hypothesis (Root 1973, Russell 1989). The resource concentration hypothesis is based on the assumption that specialist herbivores accumulate in dense patches of their host plants and reside there if the conditions are favourable (Root 1973). In diverse plant communities, individual plant species are often less prone to herbivore infestation, because host-finding is hindered due to lower host plant densities. Plants growing in small patches of low abundance appear to be structurally or chemically masked by their surrounding neighbours (Mauchline et al. 2005, Karban 2007). According to the enemies hypothesis, a diverse matrix of flowering plants in species-rich assemblages offers alternative prey, accessory food (e.g. pollen, nectar) and various shelter options for predators and parasitoids (Root 1973, Russell 1989, Jactel et al. 2005). This increased structural diversity enhances natural enemy abundance and functional diversity, finally resulting in effective biological control of specialist herbivores. 
More recently, a mechanism called associational susceptibility (White \& Whitham 2000) has been suggested to explain why in some studies no reduction, or even an increase in herbivory with increased plant diversity was found. According to this idea, generalist herbivores are thought to spill over from preferred plant species to less favoured hosts in the adjacent neighbourhood (Jactel et al. 2005, Carnus et al. 2006).

In diversity gradients across forest ecosystems, most studies carried out so far primarily focused either on specific forest pests (e.g. Su et al. 1996, Jactel et al. 2002) or generalist insect herbivores (Vehviläinen et al. 2006). Predator abundance was not included in these investigations, although it is sometimes referred to as a possible explanation for observed differences in herbivore damage (Su et al. 1996), and has only recently gained more interest in studies of forest herbivory (Jactel et al. 2006, Vehviläinen et al. 2008).

The impact of herbivore damage on plant survival is strongest in early developmental stages (Maron 1997, Hanley \& Fegan 2007), and during ontogeny defensive plant traits are subject to change (Boege \& Marquis 2005). In forest ecosystems, most studies have focused on herbivore damage in the canopy tree layer, but naturally grown saplings have rarely been used as target organisms for observation. Although early-stage tree damage caused by large herbivores (i.e. deer browsing) has been intensively investigated (Hester et al. 2000), data are scarce for insect herbivory. Studies usually only include low hanging branches of trees and larger saplings (e.g. Le Corff \& Marquis 1999, Forkner et al. 2006), rather than surveys of whole saplings in an early stage of regeneration. For juvenile trees at this stage, only data for experiments with planted trees exist (Ladd \& Facelli 2005, Löf et al. 2005, Massey et al. 2006, Norghauer et al. 2008), while in situ observations of individuals already established in the natural forest environment are so far missing. Although experiments with

planted trees can be of great value for manipulating diversity per se, they are insufficient in imitating the age structure and spatial heterogeneity of the natural forest canopy and understory (Leuschner et al., in press).

If trees are damaged by herbivores, growth and productivity of infested individuals is either reduced, eventually leading to a disadvantage in competition, or reinforced by overcompensation (Ayres et al. 2004, Zeide \& Thompson 2005, Huttunen et al. 2007). Damage may also enhance vulnerability to fungal or bacterial pathogens (Kluth et al. 2001). Insect herbivory on saplings might, hence, affect forest regeneration and play an important role in the establishment of future forest communities. No studies have been published so far on sapling herbivory and tree diversity in exclusively deciduous forests of the temperate climate zone. In particular, it is remarkable that no studies have investigated this relationship 
using Fagus sylvatica L., which has been declared to be "the most successful Central European plant species" in manner of expansion across the continent (Leuschner et al. 2006). In this study, we addressed this research gap by examining tree diversity effects on herbivory of young instead of mature trees and also included a survey of invertebrate herbivores predators. We used a gradient ranging from simple beech to diverse forest stands within a temperate, deciduous, semi-natural forest in Central Europe, and studied leaf damage in beech and maple saplings across this gradient. We hypothesised that herbivory rates decrease with increased tree diversity due to host-finding limitations, and that relative abundance of predators increases, thereby enhancing top-down control of herbivorous insects. We also tested for host plant specific differences in herbivore and predator responses. Specifically, we addressed the following questions: (1) Which sapling species is affected most by leaf-chewing insects, and how severe is the extent of leaf area loss? (2) Are diverse forest stands less susceptible to insect herbivores than simple stands? (3) How is the frequency and distribution of galls and mines across the gradient? (4) How is the relative abundance of predators and parasitoids related to tree diversity and the herbivore damage patterns?

\section{Materials and Methods}

\section{Study area}

The study was carried out in Germany's largest remaining connected semi-natural deciduous forest, the Hainich National Park, Thuringia. The Hainich forest covers a total area of 16,000 ha. Approximately half of it was designated national park in 1997 (Nationalpark Hainich; http://www.nationalpark-hainich.de).

All research plots were located in the north-eastern part of the protected area close to Weberstedt (approximately $51^{\circ} 1^{\prime} \mathrm{N}, 10^{\circ} 5^{\prime} \mathrm{E}$ ) and the average distance between plots was 4.9 $\mathrm{km}$. The study site had an elevation of $350 \mathrm{~m}$ a. s. 1 . and a temperate climate, with an average temperature of $+7.5^{\circ} \mathrm{C}(1973-2004$, Deutscher Wetterdienst). Average annual temperature in 2006 was $9.4{ }^{\circ} \mathrm{C}$ (Meteomedia 2006). Mean annual precipitation is $590 \mathrm{~mm}$ (1973-2004, Deutscher Wetterdienst), and was $518 \mathrm{~mm}$ in 2006 (Meteomedia 2006). The predominant soil type was stagnic luvisol on loess-limestone as parent material.

Plots were established across an existing gradient of canopy tree diversity ranging from simple beech to mixed forest stands with a varying number of deciduous tree species (Appendix S1). The mild climate in the area favours a wide variety of deciduous trees. The dominant tree species in the Hainich forest are Fagus sylvatica L. (Fagaceae), Tilia 
platyphyllos Scop., Tilia cordata Mill. (both Tiliaceae) and Fraxinus excelsior L. (Oleaceae). The species Carpinus betulus L. (Betulaceae), Acer campestre L., Acer platanoides L., Acer pseudoplatanus L. (all Aceraceae), Prunus avium (L.), Sorbus torminalis L. (both Rosaceae), Quercus robur L. (Fagaceae), and Ulmus glabra Huds. (Ulmaceae) occur less frequently. Coniferous trees such as Abies alba Mill., Picea abies (L.) H. Karst., Pinus sylvestris L. and Larix decidua Mill. (all Pinaceae) are scarce. All conifers are logged to regain a late successional stage of deciduous forest throughout the protected area.

\section{Site selection, assessment of herbivore damage and insect communities}

Nine observation sites were established in spring 2005. To ensure comparability, all plots were selected to share equal major stand characteristics, such as stagnic luvisol on loess soils, flat elevation, absence of canopy gaps, and have not been logged for at least 40 years. On each site, an observation plot measuring 50 x 50 m was fenced with a $2 \mathrm{~m}$ tall fence constructed of narrow mesh wire and wooden and steel pickets. The fence was build to exclude browsing and trampling by ungulates (e.g. wild boars, fallow deer, roe deer and red deer). To exclude small mammals like rabbits and hares from accessing the observation sites, the lower part of the mesh-wire was burrowed into the soil. To ensure that observed leaf damage was caused by invertebrates only, the exclosures where installed in 2005, one year prior to estimation of herbivory. The exclosures were effective in excluding large and small herbivorous mammals (ungulates, rabbits and hares) and vertebrate predators (i. e. foxes) from the plots (no signs of browsing, no scat found in 2006).

All herbivory related parameters were assessed in late June and late August 2006. This timing was chosen to allow for a sufficient time span since leave flush in early May, in which measurable herbivore damage levels could develop. Prior to June, herbivore damage was observed only exceptionally and hence not estimated. Species identity and relative abundance of tree saplings were recorded in six random 5 x 5 m subplots inside each 50 x 50 m main plot (Mölder et al. 2009), while herbaceous plant species were identified in the 5 x $5 \mathrm{~m}$ subplots and additionally in a larger 20 x $20 \mathrm{~m}$ subplot per 50 × $50 \mathrm{~m}$ plot (Mölder et al. 2006, 2008). Saplings (young trees $<75 \mathrm{~cm}$ tall) of common beech (Fagus sylvatica), Norway maple (Acer platanoides) and sycamore maple (Acer pseudoplatanus) were examined for signs of invertebrate herbivory (leaf area loss caused by leaf-chewing insects, presence of galls and mines). The three species were abundant on all plots, but the proportion of beech saplings declined with increased canopy tree diversity, while the abundance of Norway maple increased and the abundance of sycamore maple remained constant (Appendix 5.1, 5.2). 
Diversity of tree saplings was marginally correlated with canopy tree diversity (Pearson's $\rho=0.66, p=0.055$ ). To meet the prerequisites of stratified random sampling, each $50 \times 50 \mathrm{~m}$ plot was equally divided in four quarters, and ten individuals of each sapling species were randomly drawn from these subplots. Hence, on each plot, 120 saplings (= 40 per species) were inspected for leaf area loss and presence of galls and mines. An exception was made for Norway maple, which was absent on one plot and, hence, not sampled on this plot.

In total, 1,040 saplings (13,728 leaves) were examined. Leaf area loss caused by leaf-chewing invertebrates was estimated in situ by visual inspection of each single leaf using percentage score classes modified after Wint (1983); $0=$ no damage, $1=1-5 \%, 2=6-30 \%, 3=31-50$ $\%, 4=51-70 \%, 5=71-90 \%, 6=90-100 \%$. Damage rates were calculated per sapling by summing up the score class means for each leaf. The sum was then divided by the number of leaves per sapling. We furthermore punched eight leaves of every tree species per plot with a hole puncher in June 2006. The length of the leaves, of two undamaged control leaves and the diameter of the holes were measured. Measurements were repeated in August 2006. The results showed that leaf growth did not affect the extent of leaf area loss (data available upon request).

Furthermore, the presence or absence of galls and mines was noted for each leaf and damage was expressed as proportion of infested leaves per plant. Additionally the number of leaves, sapling complexity (= number of primary branches), sapling height and percentage vegetation cover in a $1 \times 1 \mathrm{~m}$ sampling quadrat surrounding the sapling were recorded. On each plot, abundance and species identity was assessed for all canopy trees with a diameter breast height $(\mathrm{dbh})>7 \mathrm{~cm}$.

Each sapling used for the estimation of herbivory was also carefully inspected for the presence of invertebrates, which were identified in situ to at least order level. For a more comprehensive survey of the invertebrate fauna present, directly adjacent to each plot 30 beating samples (10 sapling replicates of each sapling species) were taken from randomly chosen saplings to ensure that the fauna on the research plots remained undisturbed. Beating samples were obtained by beating the saplings with a wooden club against a fabric funnel attached to a collecting jar. This resulted in a total of 520 beating samples. Samples were stored at $-19 \mathrm{C}^{\circ}$ until processing.

\section{Sample processing and data analyses}

Invertebrates were separated from plant material and debris and then preserved in $70 \%$ ethyl alcohol. When species level identification was impossible, individuals were assigned to the 
lowest possible taxonomic level (at least to order). All specimens, including the individuals observed in situ, were either grouped into the feeding guilds 'chewers', 'suckers', 'predators', and 'parasitoids', or classified as 'others' (Appendix 5.3). The two groups of natural enemies, predators and parasitoids, were combined for further analyses and are for simplification hereinafter referred to as predators.

Data were analysed using the statistical software package R 2.6 (R Development Core Team 2007; http://www.R-project.org). Normality of raw data was evaluated with Shapiro-Wilk tests followed by arcsine square-root transformation of all proportion data prior to further analyses. To analyse whether tree species generally differed in leaf area loss, one-way analysis of variance (ANOVA) was performed with tree species as a categorical explanatory variable, and a Tukey's honestly significant difference test (Tukey HSD) as a post-hoc comparison.

To account for individual proportions of tree species as well as for the number of species (Magurran 2004), canopy tree diversity of the 9 research plots was expressed as Shannon index based on stem counts of all individuals with a dbh $>7 \mathrm{~cm}$. Tree diversity a priori influences a range of other abiotic and biotic plot conditions (Mölder et al. 2006), and some of them might also impact the observed herbivory patterns. No relationship with photosynthetic active radiation (PAR) was found, but soil $\mathrm{C} / \mathrm{N}$ ratios were higher in simple beech stands, whereas the number of molluscs was higher in the most diverse forest stands (Appendix S2).

Linear mixed effects models (LMEs; Pinheiro and Bates, 2000) with leaf area loss, frequency of mines, frequency of galls and predator abundance as response variables were fitted separately for all three tree species using maximum likelihood. The significance of the fixedeffect terms in each model was tested using conditional $\mathrm{F}$ tests with terms tested sequentially in the order in which they had been added to the model. LMEs are advantageous compared to simple ANOVA models because they allow the inclusion of multiple nested random effects terms to account for temporal and/or spatial pseudoreplication. Before construction of the maximal model, explanatory variables (Shannon index, number of sapling tree species, number of herbaceous plant species, $\mathrm{PAR}$, soil $\mathrm{C} / \mathrm{N}$, proportion of tree species in the canopy, proportion of tree species in the understory, sapling height, sapling complexity) were analysed for correlations, and all variables with a pairwise correlation coefficient $\geq 0.6$ were not included within the same model to minimize possible effects of multicollinearity. Shannon index was introduced as fixed variable after controlling for the variance explained by sapling complexity (number of primary branches). The maximal model was fitted with the following 
sequence: fixed effects $=$ date, sapling complexity, Shannon index, Shannon index x date; random effects were included as differences between slopes (dates) and intercepts (plots). After fitting the maximal model, model simplification was accomplished by stepwise deletion of non-significant terms based on differences in Akaike's Information Criterion (AIC) (Crawley 2007). AIC measures the lack of fit of the model; the model with the lower AIC is to be preferred (Burnham and Anderson 2002). If the difference in AIC between two models was smaller than two, empirical support for the model with the lower AIC was assumed to be substantially better (Burnham and Anderson 2002). Results for each response variable and tree species are presented in Table 5.1. To ensure that the assumptions of all tests were met, model residuals were inspected for normality and homogeneity of variances.

\section{Results}

\section{Leaf area loss of tree saplings}

By the end of the observation season, leaf area loss was generally higher in both maple species compared to beech (ANOVA, $F_{2,23}=61.18, p<0.001$, Fig. 5.1). Beech saplings had an average loss of $1.5 \%$ leaf area per leaf, whereas leaf area loss was more than twice as high in Norway maple $(3.6 \%$, Tukey HSD, $\mathrm{p}<0.001)$. Leaf area loss in sycamore maple was highest (five times higher compared to beech, $7.6 \%$, Tukey HSD, p < 0.001).

Damage increased with season $\left(\mathrm{LME}, \mathrm{F}_{1,8}=49.15, \mathrm{p}<0.001\right)$ and the response pattern remained constant in June and August. In August leaf area loss was roughly $50 \%$ lower in the most species rich stands compared to the simple beech stands (Fig. 5.2a). Relating the damage to canopy tree diversity, leaf area loss on beech declined with increased tree species richness (LME, $F_{1,7}=16.6, p=0.005$, Fig. 5.2a). No diversity-related pattern was found for the two maple species, but leaf area loss in sycamore maple was positively related to sapling complexity (LME, $\mathrm{F}_{1,7}=26.59, \mathrm{p}=0.001$, Table 5.1). 


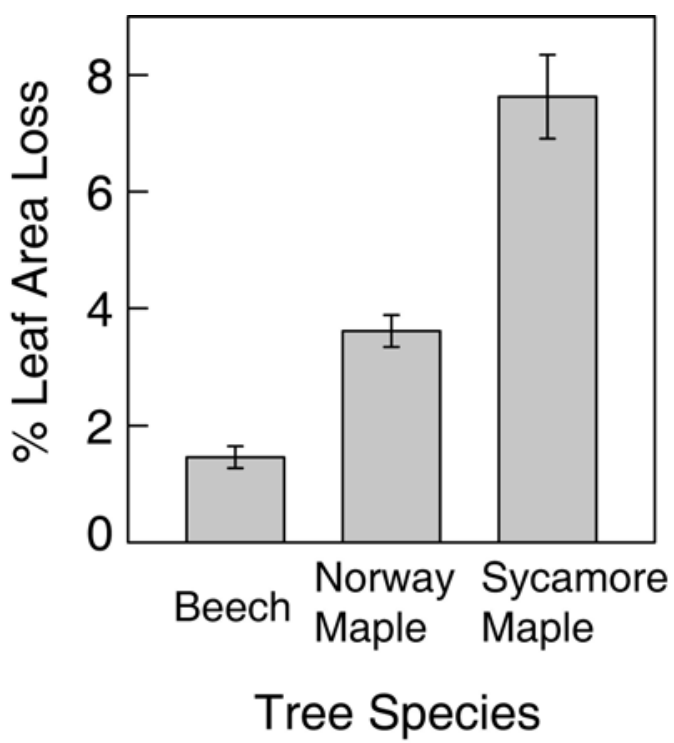

Fig. 5.1. Leaf area consumed per leaf by leaf-chewing insects on tree saplings in the Hainich National Park as mean percentages \pm one standard error (one-way ANOVA, $\mathrm{df}=2$, sum of squares $=0.1$, mean of squares $=$ $0.06, \mathrm{~F}_{2,23}=61.18, \mathrm{p}<0.001$ and Tukey HSD test, $\mathrm{p}<0.001$ for all combinations). 
Table 5.1. Simplified linear mixed effects models performed on different types of leaf damage and percent predator abundance on three tree sapling species on forest plots in the Hainich Nationalpark. $\uparrow=$ removed during model simplification; not tested $=$ no observations made for response variable. NumDF $=$ numerator degrees of freedom , DenDF $=$ denominator degrees of freedom.

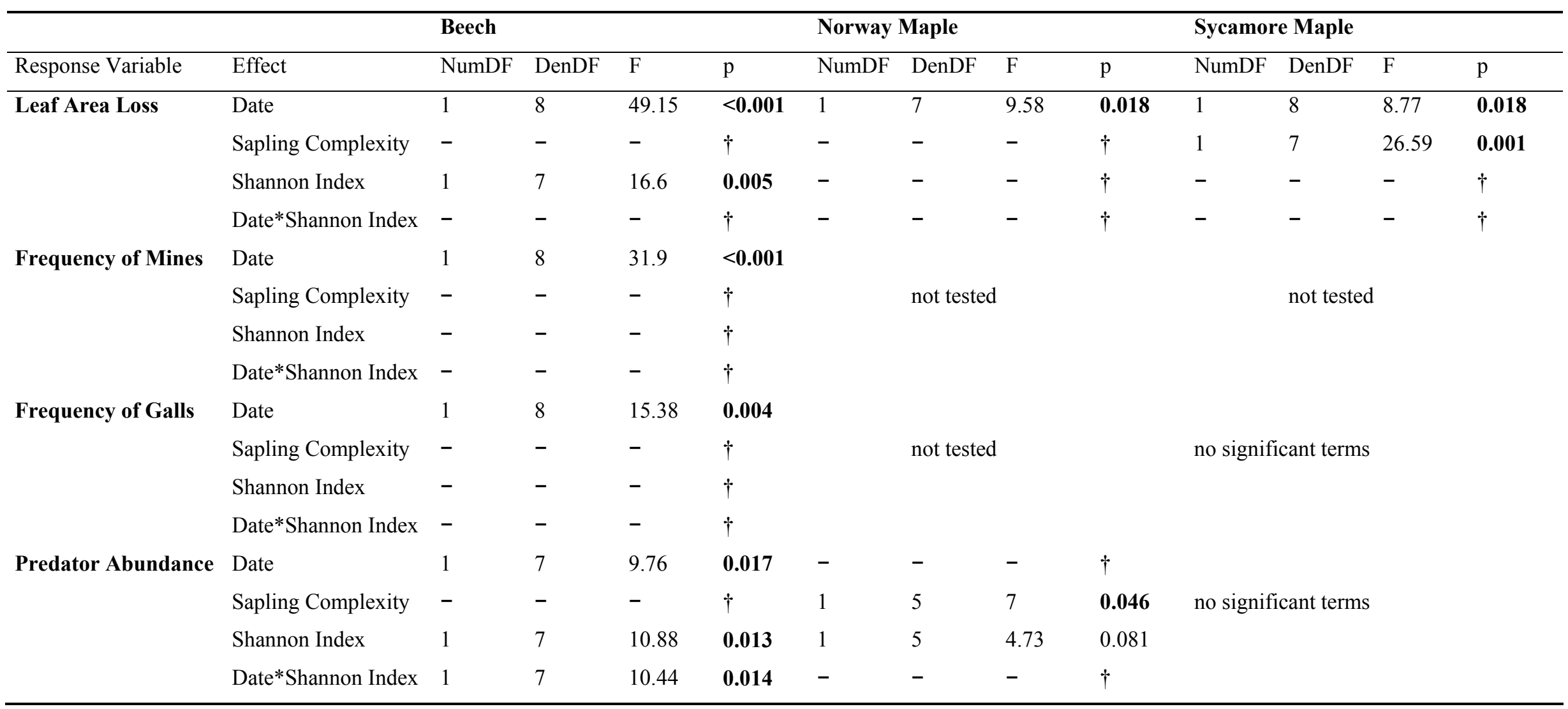




\section{Frequency and distribution of mines and galls}

Mines were only observed on beech, while galls were abundant on beech and sycamore maple. Mines surveyed on beech were caused by larvae of two species of microlepidopteran moths, Stigmella hemagyrella Kollar and Stigmella tityrella Stainton (Lepidoptera: Nepticulidae). The percentage of leaves infested with mines of Stigmella sp. increased during the season from less than $0.3 \%$ to $7.2 \%$ (LME, $F_{1,8}=31.9, p<0.001$, Fig. 5.2b). In the two most diverse forest stands, there was hardly any increase in mine frequency from June to August. Mine frequency did not correlate with canopy tree diversity (Table 5.1).

Galls on beech saplings were induced by two gall forming midges (Diptera: Cecidomyiidae), Hartigiola annulipes Hartig and Mikiola fagi (Hart.). The percentage of leaves infested with galls was higher in August compared to June (LME, $\left.F_{1,8}=15.38, p=0.004\right)$ and the overall pattern of distribution was similar for both months (Fig 5.2c). By the end of the season, gall frequency ranged from 0.1 up to $7.9 \%$ infested leaves, but was not related to canopy tree diversity. Galls on sycamore maple were induced by the gall-forming mite Aceria macrorhynchus Nalepa (Acari: Eriophyidae), but showed no response to the factors tested (Table 5.1).

Table 5.2. Number of specimens and relative abundance ( $\%$ of total) of different insect feeding guilds, sampled or observed on forest plots in the Hainich National Park. ${ }^{a}$ The sum of the percentages falls below $100 \%$ due to rounding.

\begin{tabular}{lllll}
\hline & June & August & Total & $\%$ \\
\hline Leaf chewers & 36 & 81 & 117 & 4.5 \\
Leaf suckers & 164 & 334 & 498 & 19.2 \\
Predators & 191 & 774 & 965 & 37.2 \\
Parasitoids & 51 & 51 & 102 & 3.9 \\
Other & 221 & 690 & 911 & 35.1 \\
\hline Total & & & 2593 & $99.9^{\mathrm{a}}$ \\
\hline
\end{tabular}




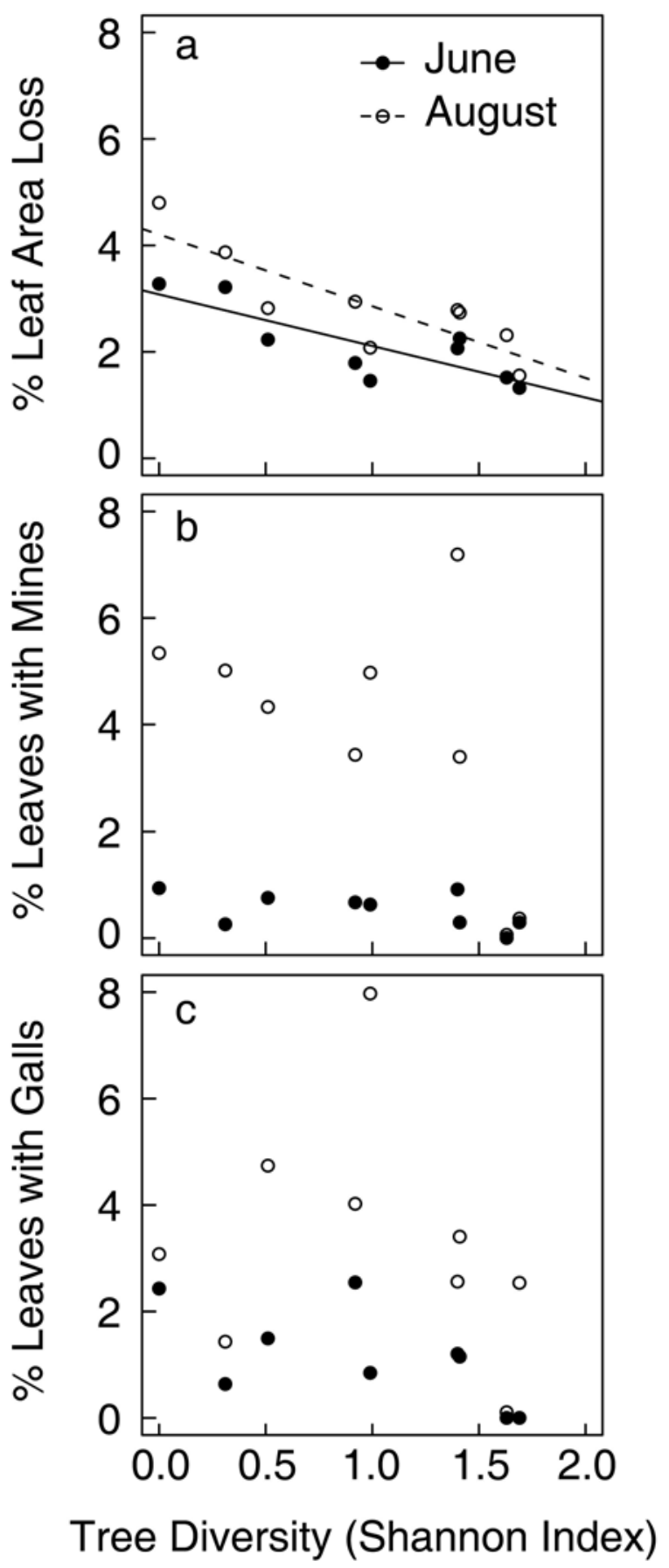

Fig. 5.2. Leaf damage of beech saplings (means per plot) across a tree diversity gradient ranging from monospecific beech to mixed forest stands in the Hainich National Park. (A) Percentage leaf area loss per leaf caused by leaf-chewing insects; (B) percentage of leaves infested with mines of Stigmella sp.; (C) percentage of leaves infested with galls of Hartigiola annulipes and Mikiola fagi. . Closed points $=$ June, open points = August. 


\section{Arthropod Community structure}

In total, 2,593 arthropods were counted across all plots. Herbivorous and predatory species were grouped into four feeding guilds: leaf chewers, leaf suckers, predators and parasitoids (Appendix 5.1). No adult leaf miners or gall formers were observed. Of all guilds, predators were most abundant (37.2\%), while leaf suckers were the most abundant herbivore feeding guild (19.2\%, Table 5.2). Parasitoids contributed $3.9 \%$ and leaf-chewing insects amounted to $4.5 \%$ of the total community (Table 5.2). Identified individuals that do not feed on trees, as well as predators that only prey on minute prey (e.g. predatory mites), were excluded from further analyses.

Both date $\left(\mathrm{LME}, \mathrm{F}_{1,7}=9.76, \mathrm{p}=0.017\right)$ and canopy tree diversity $\left(\mathrm{LME}, \mathrm{F}_{1,7}=10.88, \mathrm{p}=\right.$ 0.013) affected relative abundance of predators and parasitoids on beech saplings, but responses differed depending on observation of month (significant date * Shannon index interaction, $\mathrm{LME}, \mathrm{F}_{1,7}=10.44, \mathrm{p}=0.014$, Fig. 5.3). In June, the percentage of predators increased with increasing tree diversity by roughly 1/3, but in August the abundance of predators and parasitoids decreased with increased tree diversity. The relative abundance of predators and parasitoids was generally high (ranging from $63.6 \%$ to $100 \%$ of the total community, Fig. 5.3).

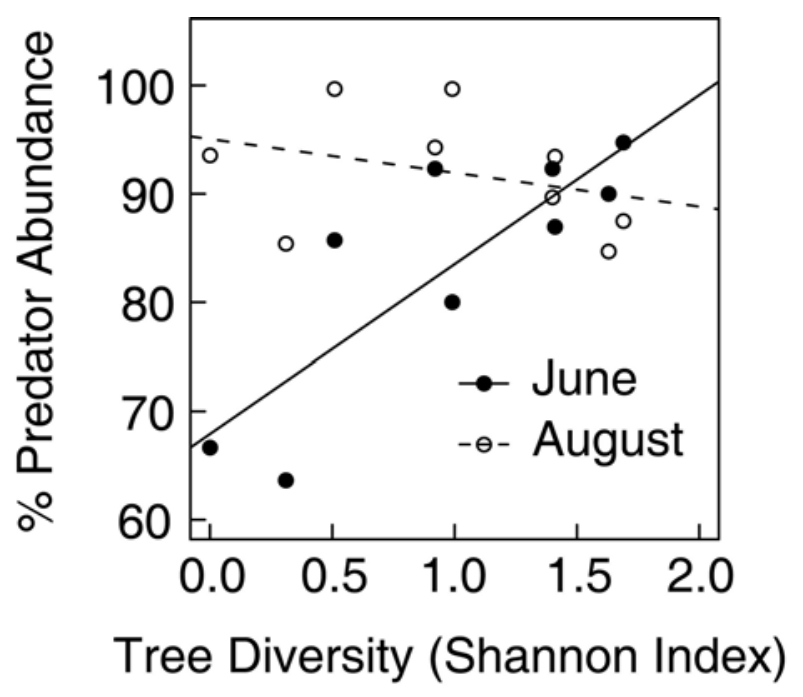

Fig. 5.3. Relative abundance of predators and parasitoids (per plot) on beech saplings across a tree diversity gradient ranging from monospecific beech to mixed forest stands in the Hainich National Park. Closed points = June, open points $=$ August. 
No significant pattern was found for the relative abundance of predators and parasitoids on sycamore maple. The arthropod community on Norway maple was not affected by canopy tree species richness $\left(\mathrm{LME}, \mathrm{F}_{1,5}=4.73, \mathrm{p}=0.081\right.$, Table 5.1), but related to sapling complexity (LME, $\mathrm{F}_{1,5}=7, \mathrm{p}=0.046$, Table 5.1).

\section{Discussion}

Our data support the hypothesis that tree saplings in diverse forest stands are less susceptible to herbivory, but the response was strongly dependent on tree species identity, as well as on herbivore feeding guild. This result is consistent with findings in the boreal zone where no general reduction of herbivore damage in the canopy of mixed forest stands was observed (Koricheva et al. 2006, Vehviläinen et al. 2006, Vehviläinen et al. 2007). Vehviläinen et al. (2006) suggest that higher quantities of deciduous trees in conifer forests may explain why in some species-rich stands herbivore damage is not reduced as hypothesised. Deciduous trees have been found to attract more generalist herbivores than conifers. Due to possible dispersal of these generalists across various neighbouring tree species, herbivory rates in forest stands with a higher proportion of deciduous trees might show only subtle or no responses to increased tree species richness (Jactel et al. 2005). Our results do not fully support this assumption. We still found a decrease of leaf area loss on beech saplings, with beech saplings and mature beech trees decreasing in abundance across the deciduous tree diversity gradienta pattern, which has been explained by mechanisms such as resource concentration (Tahvanainen \& Root 1972, Root 1973). The Janzen-Connell hypothesis (Janzen 1970, Connell 1971) also predicts that survival of tree saplings improves with increased parental distance, because herbivores more easily disperse to conspecific saplings growing in dense patches, especially near parent trees. One of the prerequisites to apply these hypotheses is that the herbivores in question are specialists (Risch et al. 1983). The few leaf-chewing species identified in our study feed on various tree species, and cannot be categorised as specialists in the narrow sense of feeding only on one host plant. Nevertheless, some of these species such as Dasychira pudibunda (Lepidoptera: Lymantriidae) show a strong host preference for beech (Schwenke 1978). Such ecological preferences, rather than strict resource specialisation, might have effectively protected beech saplings from leaf area loss in species-rich forest stands.

Our results for miners and gall formers, though specialised insects, showed no evidence for associational resistance. Vehviläinen et al. (2007) suggest feeding preferences as a possible 
explanation for observed declines of leaf miners in boreal mixed stands, but did not find the same response for gall-forming and leaf-chewing insects. The inconsistent results for chewers, leaf miners and gall-forming insects in different forest types imply that feeding specialisation may be just one of several mechanisms shaping herbivory-tree diversity relationships.

Seasonality and population dynamics after initial colonisation may be important (Otway et al. 2005), but damage patterns in our study were consistent for all observed guilds at both observation dates. This is contrary to observations on deciduous trees in other forest ecosystems, where diversity effects for miners and leaf-chewing insects were more apparent early in the year and then reversed (Vehviläinen et al. 2007).

Examples for in situ top-down control of insect herbivores by predators in forest ecosystems of different diversity are scarce (Riihimaki et al. 2005), but have been shown in experiments (Jactel et al. 2006, Kaitaniemi et al. 2007). However, recent evaluations question the relevance of the enemies hypothesis in forest ecosystems, and relate predator abundance to the presence of certain tree species rather than to tree diversity as such (Schuldt et al. 2008, Vehviläinen et al. 2008). Here, predator abundance on beech saplings was high and increased in the species rich forest stands in June when the leaves where young. This supports the enemies hypothesis (Root, 1973; Russell 1989) and suggests a diversity-related seasonal increase of top-down control, similar to observations in other ecosystems (Schmitz et al. 2000, Schmitz 2003).

Besides insect herbivores, molluscs also play an important role as herbivores in forest systems (Jennings \& Barkham 1975a, Jennings \& Barkham 1975b, Cote et al. 2005), and particularly maple is a known food resource for snails and slugs (Pigot \& Leather 2008). Herbivory patterns in our examination could not be related to the abundance of snails and slugs at the time of observation, but the higher abundance of molluscs on the most species-rich plots (Appendix S2) might be one reason why herbivore damage on maple was not affected by tree diversity. Both maple species were generally more attractive to invertebrate herbivores than beech, but data explaining why beech seems to be less palatable compared to other tree species are scarce. Further investigations should aim to test for species-specific defence mechanisms (e.g. phenolics, condensed tannin and toughness of leaves) or differences in nutrient availability when growing under the same conditions. Norway maple has previously been shown to have a high $\mathrm{N}$ content and thus a narrow $\mathrm{C} / \mathrm{N}$ ratio in the foliage (Hilfreich 1991), which has been used to explain higher herbivore damage (Brötje \& Schmidt 2005). The soil $\mathrm{C} / \mathrm{N}$ ratios observed in our study were slightly increased in the simple beech stands, which was contrary to the observed higher herbivore load for beech on these plots. In 
addition, this should affect all tree species alike, unless differences exist in soil nutrient uptake and utilisation in the plant. Light availability was very low in all stands $(0.7-2.7 \%$ of daylight intensity) and insufficient for further height growth of regeneration. Only the growth of Fagus sylvatica and Fraxinus excelsior was slightly sensitive to the marginally fluctuating irradiation (Mölder et al. 2009). Herbivores have previously been shown to be more active in forest gaps (Norghauer et al. 2008). But even though the simple beech stands we studied had higher PAR transmissivity by trend (Mölder et al. 2009), in these stands only beech saplings suffered more herbivore damage compared to saplings in the rather darker mixed forest stands. Differences in light availability amongst our forest stands might be too subtle to have any general effect on sapling herbivory, since we did not investigate fully sun-exposed gaps like Norghauer et al. (2008).

In conclusion, our study reveals new insights into the effects of tree diversity on levels of herbivory, herbivore and predator abundances in selected tree species at the sapling stage. Tree diversity has only limited explanatory power for the observed patterns in herbivory or insect abundances. By contrast, the identity and abundance of the observed tree species and seasonal effects were much better predictors in many cases. Hence, according to our results, species identity effects may be more important than species diversity per se. While it is difficult to draw such strong conclusions from observational studies alone, it will be interesting to see if tree diversity experiments simulating temperate deciduous forests such as the BIOTREE experiment (Scherer-Lorenzen et al. 2007) will show similar trends.

\section{Acknowledgements}

Financial support was obtained by the DFG [German Research Foundation] within the framework of the Research Training Group "Graduiertenkolleg 1086: The role of biodiversity for biogeochemical cycles and biotic interactions in temperate deciduous forests“. We thank Christoph Leuschner, Frank Thomas, Hermann Jungkunst and Stefan Fleck for coordination of the Research Training Group and also acknowledge Mascha Jacob, Karl M. Daenner, Anja Guckland and Andreas Mölder for providing data on biotic and abiotic plot characteristics. We thank Kyle Rea for proof-reading and 2 anonymous reviewers for helpful comments on the manuscript. All examinations complied with the current law in the country of performance. 


\section{References}

Andow D.A. (1991) Vegetational Diversity and Arthropod Population Response. Annual Review of Entomology 36, 561-586.

Ayres E., Heath J., Possell M., Black H.I.J., Kerstiens G., \& Bardgett R.D. (2004) Tree Physiological Responses to Above-Ground Herbivory Directly Modify Below-Ground Processes of Soil Carbon and Nitrogen Cycling. Ecology Letters 7, 469-479.

Balvanera P., Pfisterer A.B., Buchmann N., He J.S., Nakashizuka T., Raffaelli D., \& Schmid B. (2006) Quantifying the Evidence for Biodiversity Effects on Ecosystem Functioning and Services. Ecology Letters 9, 1146-1156.

Boege K. \& Marquis R.J. (2005) Facing Herbivory as You Grow up: the Ontogeny of Resistance in Plants. Trends in Ecology \& Evolution 20, 441-448.

Brötje J.-H. \& Schmidt W. (2005) Spitzahorn (Acer platanoides) im Kalkbuchenwald. Struktur, Streufall, Samenproduktion und Fraßschäden. Forst und Holz 60, 497-502.

Burnham K.P. \& Anderson D.R. (2002) Model Selection and Multimodel Interference. A Practical Information-Theoretic Approach. Springer Science \& Business Media, New York.

Cardinale B.J., Srivastava D.S., Duffy J.E., Wright J.P., Downing A.L., Sankaran M., \& Jouseau C. (2006) Effects of Biodiversity on the Functioning of Trophic Groups and Ecosystems. Nature 443, 989-992.

Carnus J.M., Parrotta J., Brockerhoff E., Arbez M., Jactel H., Kremer A., Lamb D., O'hara K., Walters B. (2006) Planted Forests and Biodiversity. Journal of Forestry 104, 65-77.

Connell J.H. (1971) On the Role of Natural Enemies in Preventing Competetive Exclusion in Some Marine Animals and in Rain Forest Trees. In: den Boer P.J. \& Gradwell G.R.(eds) Dynamics of Populations. Centre for Agricultural Publishing and Documentation, Wageningen, pp 298-312.

Cote M., Ferron J., \& Gagnon R. (2005) Invertebrate Predation of Postdispersal Seeds and Juvenile Seedlings of Black Spruce (Picea mariana) in the Boreal Forest of Eastern Canada. Canadian Journal of Forest Research-Revue Canadienne De Recherche Forestiere 35, 674681. 
Crawley M.J. (2007) The R Book. John Wiley \& Sons, Ltd, Chichester.

Forkner R.E., Marquis R.J., Lill J.T., \& Le Corff J. (2006) Impacts of Alternative Timber Harvest Practices on Leaf-Chewing Herbivores of Oak. Conservation Biology 20, 429-440.

Hanley M.E. (2004) Seedling Herbivory and the Influence of Plant Species Richness in Seedling Neighbourhoods. Plant Ecology 170, 35-41.

Hanley M.E. \& Fegan E.L. (2007) Timing of Cotyledon Damage Affects Growth and Flowering in Mature Plants. Plant Cell and Environment 30, 812-819.

Hester A.J., Edenius L., Buttenschon R.M., \& Kuiters A.T. (2000) Interactions Between Forests and Herbivores: The Role of Controlled Grazing Experiments. Forestry 73, 381-391.

Hilfreich H. (1991) Forstliches und Nichtforstliches über den Ahorn. Waldwirt 18, 55-59.

Hooper D.U., Chapin F.S., Ewel J.J., Hector A., Inchausti P., Lavorel S., Lawton J.H., Lodge D.M., Loreau M., Naeem S., Schmid B., Setala H., Symstad A.J., Vandermeer J., \& Wardle D. (2005) Effects of Biodiversity on Ecosystem Functioning: a Consensus of Current Knowledge. Ecological Monographs 75, 3-35.

Huttunen L., Niemela P., Peltola H., Heiska S., Rousi M., \& Kellomaki S. (2007) Is a Defoliated Silver Birch Seedling Able to Overcompensate the Growth Under Changing Climate? Environmental and Experimental Botany 60, 227-238.

Jactel H., Brockerhoff E., \& Duelli P. (2005) A Test of the Biodiversity-Stability Theory: Meta-analysis of Tree Species Diversity Effects on Insect Pest Infestations, and ReExamination of Responsible Factors. In: Scherer-Lorenzen M., Körner Ch., \& Schulze E.-D. (eds) Forest Diversity and Function. Temperate and Boreal Systems. Ecological Studies 176 Springer-Verlag, Berlin, pp 235-262.

Jactel H. \& Brockerhoff E.G. (2007) Tree Diversity Reduces Herbivory by Forest Insects. Ecology Letters 10, 835-848.

Jactel H., Goulard M., Menassieu P., \& Goujon G. (2002) Habitat Diversity in Forest Plantations Reduces Infestations of the Pine Stem Borer Dioryctria sylvestrella. Journal of Applied Ecology 39, 618-628.

Jactel H., Menassieu P., Vetillard F., Gaulier A., Samalens J.C., \& Brockerhoff E.G. (2006) 
Tree Species Diversity Reduces the Invasibility of Maritime Pine Stands by the Bast Scale, Matsucoccus feytaudi (Homoptera : Margarodidae). Canadian Journal of Forest ResearchRevue Canadienne De Recherche Forestiere 36, 314-323.

Janzen D.H. (1970) Herbivores and Number of Tree Species in Tropical Forests. American Naturalist 104, 501-528.

Jennings T.J. \& Barkham J.P. (1975a) Food of Slugs in Mixed Deciduous Woodland. Oikos 26, 211-221.

Jennings T.J. \& Barkham J.P. (1975b) Slug Populations in Mixed Deciduous Woodland. Oecologia 20, 279-286.

Kaitaniemi P., Riihimaki J., Koricheva J., \& Vehvilainen H. (2007) Experimental Evidence for Associational Resistance Against the European Pine Sawfly in Mixed Tree Stands. Silva Fennica 41, 259-268.

Karban R. (2007) Associational Resistance for Mule's Ears With Sagebrush Neighbors. Plant Ecology 191, 295-303.

Kluth S., Kruess A., \& Tscharntke T. (2001) Interactions Between the Rust Fungus Puccinia punctiformis and Ectophagous and Endophagous Insects on Creeping Thistle. Journal of Applied Ecology 38, 548-556.

Koricheva J., Vehviläinen H., Riihimaki J., Ruohomaki K., Kaitaniemi P., \& Ranta H. (2006) Diversification of Tree Stands as a Means to Manage Pests and Diseases in Boreal Forests: Myth or Reality? Canadian Journal of Forest Research-Revue Canadienne De Recherche Forestiere 36, 324-336.

Ladd B.M. \& Facelli J.M. (2005) Effects of Competition, Resource Availability and Invertebrates on Tree Seedling Establishment. Journal of Ecology 93, 968-977.

Le Corff J. \& Marquis R.J. (1999) Differences Between Understorey and Canopy in Herbivore Community Composition and Leaf Quality for Two Oak Species in Missouri. Ecological Entomology 24, 46-58.

Leuschner C., Fleck, S. \& Jungkunst H.F. (in press) Functional Role of Forest Diversity: Pros and Cons of Synthetic Stands and Across-Site Comparisons in Established Forests. Basic and Applied Ecology. Doi:10.1016/j.baae.2008.06.001 
Leuschner C., Meier I.C., \& Hertel D. (2006) On the Niche Breadth of Fagus sylvatica: Soil Nutrient Status in 50 Central European Beech Stands on a Broad Range of Bedrock Types. Annals of Forest Science 63, 355-368.

Löf M., Paulsson R., Rydberg D., \& Welander N.T. (2005) The Influence of Different Overstory Removal on Planted Spruce and Several Broadleaved Tree Species: Survival, Growth and Pine Weevil Damage During Three Years. Annals of Forest Science 62, 237-244.

Magurran A.E. (2004) Measuring Biological Diversity. Blackwell, Oxford.

Maron J.L. (1997) Interspecific Competition and Insect Herbivory Reduce Bush Lupine (Lupinus arboreus) Seedling Survival. Oecologia 110, 284-290.

Massey F.P., Massey K., Press M.C., \& Hartley S.E. (2006) Neighbourhood Composition Determines Growth, Architecture and Herbivory in Tropical Rain Forest Tree Seedlings. Journal of Ecology 94, 646-655.

Mauchline A.L., Osborne J.L., Martin A.P., Poppy G.M., \& Powell W. (2005) The Effects of Non-Host Plant Essential Oil Volatiles on the Behaviour of the Pollen Beetle Meligethes aeneus. Entomologia Experimentalis Et Applicata 114, 181-188.

Mölder A., Bernhardt-Römermann M., \& Schmidt W. (2006) Forest Ecosystem Research in Hainich National Park (Thuringia): First Results on Flora and Vegetation in Stands with Contrasting Tree Species Diversity. Waldökologie-Online 3, 83-99.

Mölder A., Bernhardt-Römermann M., \& Schmidt W. (2008) Herb-Layer Diversity in Deciduous Forests: Raised by Tree Richness or Beaten by Beech? Forest Ecology and Management 256, 272-281.

Mölder A., Bernhardt-Römermann M., \& Schmidt W. (2009) Vielfältige Baumschicht reichhaltige Verjüngung? Zur Naturverjüngung von artenreichen Laubwäldern im Nationalpark Hainich. Allgemeine Forst- und Jagdzeitung 180, 49-60.

Norghauer J.M., Malcolm J.R., \& Zimmerman B.L. (2008) Canopy Cover Mediates Interactions Between a Specialist Caterpillar and Seedlings of a Neotropical Tree. Journal of Ecology 96, 103-113.

Otway S.J., Hector A., \& Lawton J.H. (2005) Resource Dilution Effects on Specialist Insect Herbivores in a Grassland Biodiversity Experiment. Journal of Animal Ecology 74, 234-240. 
Pigot A.L. \& Leather S.R. (2008) Invertebrate Predators Drive Distance-Dependent Patterns of Seedling Mortality in a Temperate Tree Acer Pseudoplatanus. Oikos 117, 521-530.

Pinheiro J.C. \& Bates D.M. (2000) Mixed-Effects Models in S and S-PLUS. Springer, New York, London.

Riihimaki J., Kaitaniemi P., Koricheva J., \& Vehvilainen H. (2005) Testing the Enemies Hypothesis in Forest Stands: the Important Role of Tree Species Composition. Oecologia 142, 90-97.

Risch S.J., Andow D., \& Altieri M.A. (1983) Agroecosystem Diversity and Pest Control: Data, Tentative Conclusions, and New Research Directions. Environmental Entomology 12, 625-629.

Root R.B. (1973) The Organisation of a Plant-Arthropod Association in Simple and Diverse Habitats: the Fauna of Collards, Brassica olacea. Ecological Monographs 43, 95-124.

Russell E.P. (1989) Enemies Hypothesis: A Review of the Effect of Vegetational Diversity on Predatory Insects and Parasitoids. Environmental Entomology 18, 590-599.

Scherber C., Mwangi P.N., Temperton V.M., Roscher C., Schumacher J., Schmid B., \& Weisser W.W. (2006) Effects of Plant Diversity on Invertebrate Herbivory in Experimental Grassland. Oecologia 147, 489-500.

Scherer-Lorenzen M., Schulze E.D., Don A., Schumacher J., \& Weller E. (2007) Exploring the Functional Significance of Forest Diversity: a New Long-Term Experiment With Temperate Tree Species (Biotree). Perspectives in Plant Ecology Evolution and Systematics 9, 53-70.

Schmitz O.J. (2003) Top Predator Control of Plant Biodiversity and Productivity in an OldField Ecosystem. Ecology Letters 6, 156-163.

Schmitz O.J., Hambäck P.A., \& Beckerman A.P. (2000) Trophic Cascades in Terrestrial Systems: a Review of the Effects of Carnivore Removals on Plants. American Naturalist 155, 141-153.

Schwenke W. (1978) Die Forstschädlinge Europas. Dritter Band. Schmetterlinge. Verlag Paul Parey, Hamburg \& Berlin. 
Schuldt A., Fahrenholz N., Brauns M., Migge-Kleian S., Platner C., \& Schaefer M. (2008) Communities of Ground-Living Spiders in Deciduous Forests: Does Tree Species Diversity Matter? Biodiversity and Conservation 17, 1267-1284.

Sholes O.D.V. (2008) Effects of Associational Resistance and Host Density on Woodland Insect Herbivores. Journal of Animal Ecology 77, 16-23.

Su Q., Maclean D.A., \& Needham T.D. (1996) The Influence of Hardwood Content on Balsam Fir Defoliation by Spruce Budworm. Canadian Journal of Forest Research-Revue Canadienne De Recherche Forestiere 26, 1620-1628.

Tahvanainen J.O. \& Root R.B. (1972) The Influence of Vegetational Diversity on the Population Ecology of a Specialised Herbivore, Phyllotreta cruciferae (Coleoptera: Chrysomelidae). Oecologia 10, 321-346.

Unsicker S.B., Baer N., Kahmen A., \& Wagner M., Buchmann N., Weisser W.W. (2006) Invertebrate Herbivory Along a Gradient of Plant Species Diversity in Extensively Managed Grasslands. Oecologia 150, 233-246.

Vehviläinen H., Koricheva J., \& Ruohomäki K. (2007) Tree Species Diversity Influences Herbivore Abundance and Damage: Meta-Analysis of Long-Term Forest Experiments. Oecologia 152, 287-298.

Vehviläinen H., Koricheva J., \& Ruohomäki K. (2008) Effects of Stand Tree Species Composition and Diversity on Abundance of Predatory Arthropods. Oikos OnlineEarly Articles, doi: 10.1111/j.0030-1299.2008.15972.x.

Vehviläinen H., Koricheva J., Ruohomäki K., Johansson T., \& Valkonen S. (2006) Effects of Tree Stand Species Composition on Insect Herbivory of Silver Birch in Boreal Forests. Basic and Applied Ecology 7, 1-11.

White J.A. \& Whitham T.G. (2000) Associational Susceptibility of Cottonwood to a Box Elder Herbivore. Ecology 81, 1795-1803.

Wint G.R.W (1983) Leaf damage in tropical rain forest canopies. In: Sutton S.L., Whitmore T.C., \& Chadwick A.C. (eds) Tropical Rain Forest: Ecology and Managemen.t British Ecological Society, Blackwell Scientific, Oxford, pp 229-239.

Zeide B. \& Thompson L.C. (2005) Impact of Spring Sawfly Defoliation on Growth of 
Loblolly Pine Stands. Southern Journal of Applied Forestry 29, 33-39. 


\section{Appendix 5.1}

Relative abundance of trees in the canopy (C, per plot) and saplings in the understory (U, per ha; see Mölder et al. 2009) on 9 deciduous forest plots of different diversity in the Hainich National Park.

\begin{tabular}{|c|c|c|c|c|c|c|c|c|c|c|c|c|c|c|c|c|c|c|}
\hline \multirow{2}{*}{$\begin{array}{l}\text { Shannon Index } \\
\text { Stratum }\end{array}$} & \multicolumn{2}{|l|}{0} & \multicolumn{2}{|l|}{0.31} & \multicolumn{2}{|l|}{0.51} & \multicolumn{2}{|l|}{0.99} & \multicolumn{2}{|l|}{0.92} & \multicolumn{2}{|l|}{1.4} & \multicolumn{2}{|l|}{1.41} & \multicolumn{2}{|l|}{1.69} & \multicolumn{2}{|l|}{1.63} \\
\hline & $\mathrm{C}$ & $\mathrm{U}$ & $\mathrm{C}$ & $\mathrm{U}$ & $\mathrm{C}$ & $\mathrm{U}$ & $\mathrm{C}$ & $\mathrm{U}$ & $\mathrm{C}$ & $\mathrm{U}$ & $\mathrm{C}$ & $\mathrm{U}$ & $\mathrm{C}$ & $\mathrm{U}$ & $\mathrm{C}$ & $\mathrm{U}$ & $\mathrm{C}$ & $\mathrm{U}$ \\
\hline \multicolumn{19}{|l|}{$\%$} \\
\hline Acer campestre & - & & - & - & - & 0.2 & - & - & - & - & - & - & - & - & 2 & 1.4 & - & 0.4 \\
\hline Acer platanoides & - & - & - & 20.9 & 1.9 & 6.3 & 0.8 & 61.0 & 1 & 34.4 & 3.7 & 40.0 & 0.9 & 32.6 & 4.1 & 28.2 & 6 & 65.2 \\
\hline Acer pseudoplatanus & - & 20.3 & 1.9 & 18.0 & - & 15.2 & 1.5 & 11.0 & 1.5 & 19.3 & 1.8 & 23.0 & 3.4 & 33.2 & 2 & 13.4 & 1.2 & 15.8 \\
\hline Carpinus betulus & - & - & - & - & - & - & - & - & - & - & - & 1.1 & 3.4 & - & 9.2 & 1.8 & 10.8 & 1.8 \\
\hline Fagus sylvatica & 100 & 71.9 & 93.5 & 4.1 & 83.3 & 66.3 & 59.4 & 7.5 & 73.7 & 34.7 & 47.7 & 11.7 & 41.9 & 7.1 & 3.1 & 2.3 & 2.4 & 4.7 \\
\hline Fraxinus escelsior & - & 1.6 & 1.9 & 56.4 & - & 8.9 & 33.1 & 16.0 & 12.9 & 11.2 & 13.8 & 22.3 & 16.2 & 27.1 & 7.1 & 44.8 & 13.3 & 9.3 \\
\hline Prunus avium & - & 3.1 & - & - & - & - & - & - & - & - & - & - & - & - & 1 & 5.9 & - & 2.2 \\
\hline Quercus petraea & - & - & - & - & 14.8 & - & - & - & - & - & - & - & - & - & - & - & - & - \\
\hline Quercus robur & - & - & - & - & - & - & - & - & - & - & - & - & - & - & 4.1 & - & 1.2 & - \\
\hline Sorbus aucuparia & - & 3.1 & - & - & - & 0.2 & - & - & - & - & - & - & - & - & - & - & - & - \\
\hline Tilia cordata & - & - & - & - & - & 2.9 & 2.3 & 2.5 & 5.7 & 0.4 & 22 & 1.5 & 30.8 & - & 24.5 & 1.4 & 22.9 & - \\
\hline Tilia platyphyllos & - & - & 2.8 & 0.6 & - & - & 1.5 & 1.5 & 5.2 & - & 11 & 0.4 & 3.4 & - & 42.9 & 0.7 & 41 & - \\
\hline Ulmus glabra & - & - & - & - & - & - & 1.5 & 0.5 & - & - & - & - & - & - & - & 0.2 & 1.2 & 0.7 \\
\hline
\end{tabular}




\section{Appendix 5.3}

Spearman rank correlations of biotic and abiotic plot characteristics with canopy tree diversity. Significance levels: $* * * \mathrm{p}<0.001, * * \mathrm{p}<0.01, * \mathrm{p}<0.05$. ${ }^{\mathrm{C}}$ Canopy, ${ }^{\mathrm{U}}$ Understory, June, ${ }^{\mathrm{A}}$ August.

\begin{tabular}{|c|c|c|}
\hline & $\begin{array}{l}\text { Canopy Tree } \\
\text { (Shannon) }\end{array}$ & Diversity \\
\hline Tree Species Understory & 0.66 & \\
\hline Plant Species Understory & $0.94 * * *$ & \\
\hline$\%$ Beech $^{\mathrm{C}}$ & $-0.94 * * *$ & \\
\hline$\% \operatorname{Beech}^{\mathrm{U}}$ & $-0.71^{*}$ & \\
\hline$\%$ Norway Maple & $0.76^{*}$ & \\
\hline$\%$ Norway Maple ${ }^{U}$ & $0.69 *$ & \\
\hline$\%$ Sycamore Maple $^{\mathrm{C}}$ & 0.6 & \\
\hline$\%$ Sycamore Maple ${ }^{U}$ & 0.09 & \\
\hline Stem Density & 0.26 & \\
\hline PAR & -0.56 & \\
\hline Soil C/N & $-0.74 *$ & \\
\hline Snails \& Slugs ${ }^{\mathrm{J}}$ & $0.73 *$ & \\
\hline Snails \& Slugs ${ }^{\mathrm{A}}$ & 0.53 & \\
\hline
\end{tabular}




\section{Appendix 5.3}

Feeding guilds and classification of the insect community sampled/observed on forest plots in the Hainich National Park.

\begin{tabular}{|c|c|c|c|}
\hline Feeding guild & Associated taxa & Order & Family \\
\hline \multirow[t]{5}{*}{ Leaf chewers } & Butterfly larvae & Lepidoptera & Lymantriidae \\
\hline & Sawfly larvae & Hymenoptera & \\
\hline & Leaf beetles & Coleoptera & Chrysomelidae \\
\hline & Leaf weevils & Coleoptera & Curculionidae (Entiminae) \\
\hline & Short-horned grasshoppers & Orthoptera & Acrididae \\
\hline \multirow[t]{4}{*}{ Leaf suckers } & Leaf hoppers & Homoptera & Cicadellidae \\
\hline & Frog hoppers & Homoptera & Cercopidae \\
\hline & Sap-sucking true bugs & Heteroptera & \\
\hline & Aphids & Homoptera & Aphidae \\
\hline \multirow[t]{7}{*}{ Predators } & Spiders & Araneae & \\
\hline & Predatory true bugs & Heteroptera & \\
\hline & Robber flies & Diptera & Asilidae \\
\hline & Scorpion flies & Mecoptera & Panorpidae \\
\hline & Click beetles & Coleoptera & Elateridae \\
\hline & Rove beetles & Coleoptera & Staphylinidae \\
\hline & Earwigs & Dermaptera & \\
\hline Parasitoids & Parasitic wasps & Hymenoptera & \\
\hline
\end{tabular}




\section{CHAPTER}

6

Discussion \& Conclusion 


\section{Discussion \& Conclusion}

To test and unravel biodiversity-functioning relationships, currently two competing approaches exist: biodiversity experiments (e.g. Jena Experiment, Cedar Creek, BIODEPTH, BIOTREE) and real-world observations (e.g. Research Training Group 1086, Biodiversity Exploratories). The goal behind both approaches is to evaluate how plant species loss affects ecosystem functioning. Whereas biodiversity experiments might be a useful tool for investigating biodiversity-functioning relationships for assessments of productivity in grassland habitats (Schmid 2002), their explanatory value for forest ecosystems is debatable. Major concerns of applicability and comparability to real-world forest systems include the small plot size, plot history, age structure, canopy architecture and planting patterns (Leuschner et al. in press). Especially if faunal responses to plant diversity are investigated, it is questionable if the fauna residing on experimental sites is comparable to the native fauna of naturally grown vegetation, which has developed over a long time span and undergone various succesional stages. Moreover, not only the residing fauna in biodiversity experiments might be artificial, the assemblage of plant species in biodiversity experiments is strongly biased and does not necessarily correspond to the environmental conditions of real-world systems (Leps 2004), especially if multiple site comparisons are lacking. In contrast, observational studies are often criticised because they lack control and manipulation of possible confounding variables, such as soil fertility etc., which limits strong conclusions on how plant diversity per se affects higher trophic levels and ecosystem functioning. Nevertheless, this limitation can be overcome by integrating researchers of multiple disciplines into large-scale observational projects, thereby ensuring consolidation of measurements which should comprise as many biotic and abiotic parameters as possible.

In the multidisciplinary observational study on hand, species richness of beetles and true bugs responded to the semi-natural tree diversity gradient in accordance to our main hypothesis that insect species richness increases with increased tree diversity. However, species richness of cavity-nesting bees and wasps did not directly respond to tree diversity per se and only increased with enhanced bee and wasp abundance, but differences were present across forest strata. Considering the fact that different taxa depend on different resources, it is not surprising that tree diversity does not affect all taxa directly and alike. Beetles and in particular, true bugs, are often closely associated with certain plant and tree species (Wachmann et al. 2004), and should thus be more susceptible to changes in tree and plant diversity. In contrast, bees might depend on a high variety and density of flowering resources 
(Höhn et al. submitted) simply not present in temperate forest habitats, whereas wasp diversity and abundance might also be controlled by the availability of larval food (e.g. aphids, lepidopteran caterpillars, spiders) and light intensity (Klein et al. 2006).

We could demonstrate for beetles and true bugs, that species turnover ( $\beta$-diversity) in space and time contributed most to overall $\gamma$-diversity, accounting for about $90 \%$ of the observed total species richness. It was particularly striking that turnover between trees, even between conspecific beech individuals, was high if a sufficient number of tree replicates was analysed. Besides stand-level heterogeneity mediated by tree diversity, between-tree heterogeneity, e. g. different microhabitat conditions (microclimate, water-run off, sun exposure etc.) might play an important role for the diversity and distribution of insect species within temperate deciduous forest stands (Winter \& Möller 2008).

Additionally we could show that leaf area loss of beech saplings was directly related to tree diversity and decreased in the more species rich stands as hypothesised, while relative abundance of predators increased, but no effect was found for other tree species or the abundance of galls and mines. Here, mechanisms of resource concentration (Tahvanainen \& Root 1972, Root 1973,) and natural enemy abundance (Root 1973, Russell 1989) most likely play a role for biocontrol of insect herbivores, and our results demonstrate that multitrophic interactions in real-world forest ecosystems are complex and vary vastly across the studied taxa. Further studies, for example focusing on certain herbivore species and using phytometers, might help to disentangle the core mechanisms of the observed biodiversityfunctioning relationships.

We conclude that in diverse real-word forest ecosystems, a variety of biodiversity-functioning relationships are effective. A loss of tree species would result in changes of the residing insect community, leading to a shift and eventually loss of functional responses. Simple beech stands alone are insufficient in conserving forest biodiversity and the related ecosystem functions. Forest management has to ensure that a variety of deciduous tree species is afforested and conserved, thereby maintaining habitat heterogeneity. It remains tantalising if the patterns observed in our study can be reproduced and approved in temperate tree diversity experiments, and we suggest an integrative approach of real-world observations and diversity experiments as best-practice for future research. 


\section{References}

Höhn, P., Steffan-Dewenter, I., Lewis, O.T., \& Tscharntke, T. (submitted) Relative Contribution of Cacao Agroforestry, Rainforest and Openland to Local and Regional Bee Diversity.

Klein, A.M., Steffan-Dewenter, I., \& Tscharntke, T. (2006) Rain Forest Promotes Trophic Interactions and Diversity of Trap-Nesting Hymenoptera in Adjacent Agroforestry. Journal of Animal Ecology 75, 315-323.

Leps, J. (2004) What Do the Biodiversity Experiments Tell Us About Consequences of Plant Species Loss in the Real World? Basic and Applied Ecology 5 , 529-534.

Leuschner, C., Jungkunst, H.F., \& Fleck, S. (in press) Studying the Functional Role of Tree Diversity in Forests: the Pros and Cons of Synthetic Stands and Across-Site Comparisons in Established Forests. Basic and Applied Ecology.

Root, R.B. (1973) The Organisation of a Plant-Arthropod Association in Simple and Diverse Habitats: the Fauna of Collards, Brassica olacea. Ecological Monographs 43, 95-124.

Russell, E.P. (1989) Enemies Hypothesis: A Review of the Effect of Vegetational Diversity on Predatory Insects and Parasitoids. Environmental Entomology 18, 590-599.

Schmid, B. (2002) The Species Richness-Productivity Controversy. Trends in Ecology \& Evolution 17, 113-114.

Tahvanainen, J.O. \& Root, R.B. (1972) The Influence of Vegetational Diversity on the Population Ecology of a Specialised Herbivore, Phyllotreta cruciferae (Coleoptera: Chrysomelidae). Oecologia 10, 321-346.

Wachmann, E., Melber, A., \& Deckert, J. (2004) Wanzen Band 2 Goecke \& Evers, Keltern.

Winter, S. \& Moller, G.C. (2008) Microhabitats in Lowland Beech Forests as Monitoring Tool for Nature Conservation. Forest Ecology and Management 255, 1251-12 


\section{Summary}

Throughout the last decade, linking biodiversity with ecosystem functioning and services, such as pollination and biocontrol of pest species, has gained major attention in ecological research. In particular biodiversity loss due to human land use and disturbance has been determined as a major threat, eventually leading to a decline in ecosystem services and functions.

The major proportion of global biodiversity can be found in forest ecosystems, and especially in tropical regions pristine forest habitats become more and more scarce. In temperate regions the situation is hardly any better: even though in Germany $30 \%$ of land surface is covered by forest, the proportion of old-growth natural forest equals zero. Hence it is of immediate importance to identify the contribution of the remaining semi-natural forests to biodiversity and ecosystem functioning.

Investigations in tropical rainforests have shown that insects in the forest canopy contribute highly to global biodiversity, but data on canopy fauna in temperate deciduous forests are scarce. Even though plant diversity in agricultural or experimental grassland habitats has often been related to insect diversity, no information is available whether the same relationship exists in forest habitats. In the study on hand, we related diversity and function of canopy and understory insect communities to a natural tree diversity gradient ranging from simple beech to mixed deciduous forest stands. The scope of our investigation was to analyse effects of canopy tree diversity on insect species richness, community structure and multitrophic interactions across various spatiotemporal scales in a real-world ecosystem, based on two major hypotheses: (1) insect diversity increases with increased canopy tree diversity, (2) herbivory decreases with increased canopy tree diversity.

The study was conducted using 12 forest plots in the Hainich National Park, Germany's largest remaining semi-natural deciduous forest. A variety of methods was applied to sample the resident insect fauna, such as flight-interception traps, beating samples, and trap nests for cavity-nesting hymenopterans. Furthermore, herbivory of tree saplings was estimated (leaf area loss, abundance of galls and mines). This approach ensured that a variety of taxa (beetles, true bugs, bees, wasps and their natural enemies) and functions (herbivory, predation, parasitism) was included in the analyses.

Total species richness ( $\gamma$-diversity) of beetles and true bugs increased across the tree diversity gradient as hypothesised, and the same pattern was found for most functional groups (e.g forest specialists, predators etc.). Species richness of cavity-nesting bees and wasps did not 
respond to tree diversity. For beetles and true bugs, species turnover ( $\beta$-diversity) in space and time contributed most ( $\sim 90 \%$ ) to overall $\gamma$-diversity. Turnover between tree individuals was high if a sufficient number of tree replicates was analysed, even among trees of the same species in simple beech stands. Besides stand-level heterogeneity, between-tree heterogeneity apparently plays a major role in determining insect species richness in deciduous forests. Furthermore, the forest stands showed a distinct stratification of bee and wasp abundance, community composition, and parasitism rates. Enhanced resource availability (e.g. nesting sites, host and prey abundance) might be one reason why increased tree diversity also controls species richness, but not function of higher trophic levels. Concerning herbivory damage on tree saplings in the forest understory, leaf area loss was generally higher in maple compared to beech saplings, but only beech showed a decline in damage across the tree diversity gradient. No pattern was found for galls and mines. Relative abundance of predators on beech showed a seasonal response and increased on species-rich plots in June, suggesting higher biological control. Rather than tree diversity per se, the identity and abundance of the sampled tree species in most cases was a better predictor for the observed responses, and effects can be explained by mechanism of resource concentration and abundance of natural enemies (enemies hypothesis).

In conclusion, our study gives new insights into the effects of tree diversity on insect species richness and multitrophic interactions. Although planted experiments are of great value for determining fundamental mechanisms behind observed effects, these effects might not occur or run differently in undisturbed, semi-naturally established forests, since planted experiments are insufficient in imitating the age structure and spatial heterogeneity of natural forests. By studying one of Europe's largest semi-natural deciduous forests we could demonstrate that complex biodiversity-functioning relationships are effective in real-world forest ecosystems, and that temporal and spatial species turnover is the key to understanding biodiversity patterns. It became evident that simple beech stands alone are unsatisfactory in conserving the full set of regional insect species richness. We suggest that sustainable forest management should aim at maintaining a diverse mix of structurally different tree species and individuals, thereby enhancing habitat heterogeneity and providing resources for a diverse and highly functional insect community. 


\section{Zusammenfassung}

Während des letzen Jahrzehnts ist der Zusammenhang zwischen Biodiversität und Ökosystemfunktionen bzw. -dienstleistungen, wie z. B. Bestäubung oder biologische Schädlingskontrolle, ins Zentrum ökologischer Forschung gerückt. Vor allem Verlust von Biodiversität durch anthropogen bedingte Störung und Landnutzung wurde als Gefahr determiniert, wodurch ein Rückgang an Ökosystemfunkionen und -dienstleistungen zu erwarten ist. Der größte Anteil der globalen Biodiversität findet sich in Waldökosystemen, und besonders in den Tropen nimmt der Anteil an ursprünglichen Waldhabitaten immer mehr ab. Ach in der temperaten Zone ist die Situation kaum besser: Obwohl in Deutschland 30 \% der Landesfläche von Wald bedeckt sind, beträgt der Urwaldanteil an diesen Waldflächen gleich Null. Daher ist es von großer Bedeutung, den Beitrag der noch verbleibenden naturnahen Wälder für den Erhalt von Biodiversität und den damit einhergehenden Ökosystemfunktionen zu bestimmen. Untersuchungen in tropischen Regenwäldern haben gezeigt, das Insekten im Kronenraum den größten Teil der globalen Biodiversität stellen, für temperate Laubwälder liegen jedoch kaum Daten vor. Obwohl Pflanzendiversität in der Agrarlandschaft oder in experimentellen Graslandhabitaten häufig mit erhöhter Insektendiversität in Verbindung gebracht warden konnte, gibt es kaum Hinweise ob der gleiche Effekt auch in Waldhabitaten gilt. In der vorliegenden Arbeit wurde die Diversität und Funktion von Insektengemeinschaften entlang eines natürlichen Baumartendiverstätsgradienten untersucht, unter Berücksichtigung von Buchenreinbeständen bis hin zu hochdiversen Laubmischwäldern. Ziel der Arbeit war eine Analyse des Effekts von Baumartendiversität auf den Artenreichtum, die Gemeinschaftsstruktur und multitrophische Interaction von Insekten entlang verschiedener räumlich-zeitlicher Skalen in einem real existierenden (nicht experimentellen) Ökosystem. Dabei wurden folgende Leithypothesen verfolgt: (1) Insektendiversität ist mit steigender Baumartenvielfalt erhöht, (2) Herbivorieschäden werden bei zunehmender Baumartenvielfalt verringert.

Für die Untersuchung wurden 12 Waldflächen im Nationalpark Hainich, Deutschlands größtem noch verbleibenden zusammenhängenden Laubwaldgebiet, genutzt. Verschiedene Methoden kamen zum Fang der Insektenfauna zum Einsatz, z. B. Kreuzfensterfallen, Klopfproben und Nisthilfen für Bienen und Wespen. Desweiteren wurde der Herbivorieschaden von Baumjungwuchs geschätzt (Verlust an Blattfläche, Vorhandensein von Gallen und Minen). Durch diesen Ansatz konnte gewährleistet werden, dass eine Vielfalt von Taxa (Käfer, Wanzen, Bienen, Wespen und deren natürliche Feinde) und Funktionen 
(Herbivorie, Prädation, Parasitismus) in die Analyse mit einbezogen werden konnte. Der Gesamtartenreichtum ( $\gamma$-Diversität) der Käfer und Wanzen nahm mit steigender Baumartenvielfalt $\mathrm{zu}$, und das gleiche Muster konnte auch in den meisten funktionellen Gruppen gefunden werden (z. B. Waldspezialisten, Prädatoren, etc.). Der Artenreichtum von Bienen und Wespen reagierte jedoch nicht auf die erhöhte Baumartenvielfalt. Bezüglich Käfern und Wanzen spielte vor allem der räumlich und zeitliche Artenturnover ( $\beta$-Diversität) eine Rolle und trug am meisten $(\sim 90 \%)$ zur allgemeinen $\gamma$-Diversität bei. Der Turnover zwischen Baumindividuen war stets hoch, wenn eine ausreichend hohe Anzahl an Bäumen berücksichtigt wurde, auch zwischen Bäumen der gleichen Art in Buchenreinbeständen. Neben Heterogenität auf Bestandesebene spielt also auch Heterogenität zwischen Einzelbäumen eine Rolle für den Artenreichtum von Insekten in temperaten Laubwäldern. Darüberhinaus zeigten die untersuchten Bestände auch eine distinkte Stratifizierung von Bienen- und Wespenabundanz, Gemeinschaftszusammensetzung und Parasitierungsrate. Gesteigerte Ressourcenverfügbarkeit (z. B. Nistmöglichkeiten, Wirts- und Beuteabundanz), vor allem im Kronenraum ist ein möglicher Grund warum Baumartenvielfalt sich ausserdem positiv auf den Artereichtum höher trophischer Ebenen (Parasitoide) auswirkt. Eine gesteigerte Funktion (Parasitierungsrate) konnte mit dem Anstieg der Baumartenvielfalt jedoch nicht in Zusammenhang gebracht werden. Bezüglich des Herbivorieschadens an Baumjungwuchs in der Krautschicht konnte festgestellt werden, das der Blattflächenverlust zwar generell bei Ahorn am höchsten war, jeduch nur bei Buchensämlingen mit steigender Baumartenvielfalt tatsächlich auch geringer wurde. Es wurde keine Bestimmtes Muster für das Vorkommen von Gallen und Minen gefunden. Die relative Abundanz von Prädatoren auf Buchensämlingen zeigt ein saisonal bedingtes Muster und stieg im Juni in gemischten Beständen an, was eine erhöhte biologische Schädlingskontrolle bewirkt haben könnte. Nicht Baumartenvielfalt allein, sondern die Artidentität und Abundanz der beprobten Baumsämlige war Häufig besser geeignet, um die beobachteten Effekte zu erklären, welche vermutlich sich vermutlich auf Ressourcenkonzentration und die Abundanz von natürlichen Feinden zurückführen lassen.

Zusammenfassend lässt sich feststellen, das die vorliegende Arbeit neue Einsichten bezüglich des Effekts von Baumartenvielfalt auf den Artenreichtum und multitrophisch Interaktionen von Insektengemeinschaften zulässt. Auch wenn angepflanzte Experimente einen großen Wert für die Bestimmung fundamentaler Mechanismen bestimmter Effekte besitzen, kann es dennoch sein dass diese Effekte in naturnahen Wäldern anders, oder im Extremfall gar nicht, in Erscheinung treten, da Anpflanzungen die Altersstruktur und räumliche Heterogenität eines 
natürlich gewachsenen Waldes nur unzulänglich wiedergeben. Durch die Untersuchung eines der größten zusammenhängenden Laubwaldgebiete Europas konnten wir zeigen, dass in realen Waldökosystem komplexe Biodiversitäts-Ökosystemfunktionsbeziehungen eine Rolle spielen, und das räumlicher und zeitlicher Artenturnover der Schlüssel zum Verständnis von Diversitätsmustern ist. Es konnte deutlich gemacht warden, dass Buchenreinbestände nur unzulänglich in der Lage sind, den vollen regionalen Artenreichtum von Insekten zu erhalten. Wir schlagen vor, dass nachhaltige Waldnutzung darauf abzielen sollte, dass ein diverser Mix an strukturell verschiedenen Baumarten und -individuen erhalten bleibt, wodurch die Habitatheterogenität erhöht wird, un mehr Ressourcen für eine artenreiche und hochfunktionelle Insektenlebensgemeinschaft zur Verfügung steht. 


\section{Acknowledgements}

This research would have been impossible to complete without my supervisors, Prof. Dr. Teja Tscharntke and Prof. Dr. Ingolf Steffan-Dewenter, to whom I owe great debt for providing me with an interesting project, assistance, lots of scientific discussions, encouragement and for creating a friendly and enjoyable work environment! I also acknowledge Prof. Dr. Matthias Schaefer and Prof. Dr. Stefan Vidal for being part of my PhD- and/or defence committee, and Dr. Dirk Gansert and Dr. Frank Thomas for organising all matters involved with the newly established $\mathrm{PhD}$ programme "Biodiversity \& Ecology". I furthermore kindly thank Prof. Dr. Christoph Leuschner, Dr. Stefan Fleck, Dr. Herrmann Jungkunst and Dr. Frank Thomas for organisation and coordination of the Research Training Group „Graduiertenkolleg 1086: The role of biodiversity for biogeochemical cycles and biotic interactions in temperate deciduous forests". Financial support was kindly granted by the DFG [German Research Foundation] within the framework of the above mentioned Research Training Group, and I highly acknowledge all participants, supervisors and $\mathrm{PhD}$ students, for offering interesting workshops, good collaboration, data sharing and for the enjoyable time we spent together in Göttingen and the Hainich National Park. I am particularly grateful to the official management of the Hainich National Park for providing the permission to conduct research in Germany's largest remaining (and probably most beautiful) deciduous forest, which was the basis to render this project possible in the first place.

I am extremely grateful to Susanne Schiele for being the biggest help with all kinds of field work despite the obnoxious ticks, and especially for spending hour after hour in the lab's basement to dissect hundreds of trap nests. The extensive field and identification work for this thesis would not have been possible without the substantial help of Tobias Gebauer, Dr. Martin Goßner, Dr. Ulrich Simon, Boris Büche, Martin Erren, Friedrich Rathing, Andreas Dittrich, Elisabeth Nowak and Mareen Gollnow, and I am also greatly indebted to Dr. Christoph Scherber for his generous support regarding my statistical issues- you really $\mathrm{R}$ (Version 2.6.2) the best!

My greatest thanks are probably reserved for my Dad, who helped me to get through many years of happiness and after the death of my mother, often despair, and who at all times told me to never give up. If only Mum had won her battle and could witness that I eventually became what she always wished for me! I am also grateful to Uffe N. Nielsen, who finally succeeded in convincing me to believe in myself and to go for what I want, even though I still dislike going for it all by myself. Finally, I thank my friends and colleagues (also known as 
Agroecology group) for simply being around and sharing the ups and downs of completing a $\mathrm{PhD}$ degree and of life in general, especially Barbara Scheid, Tobias Gebauer and Patrick Höhn (who saved me from becoming smelly by generously providing his washing machine), and would like to bid farewell with another quote by famous Edward O. Wilson:

"Most children have a bug period, and I never grew out of mine!" 


\section{Curriculum Vitae}

Stephanie Sobek, born 28.06.1978 in Hamm (Westf.), Germany

\section{Education}

04/2005 - 09/2008 University of Göttingen, Germany Biodiversity and Ecology, $\mathrm{PhD}$ degree (Dr. rer. nat.)

05/2000 - 11/2002 University of Osnabrück, Germany

Biology, Diplom

Thesis: "Mite diversity in a Central European forest canopy" Thesis lab work was accomplished at Freie Universität Berlin, Germany, (10/2001-11/2002)

09/1999 - 04/2000 University of Victoria, Canada

Biology, exchange student

10/1997 - 08/1999 University of Osnabrück, Germany

Biology, Vordiplom

06/1988 - 06/1997 Gymnasium Hammonense (high school), Hamm, Germany.

High school diploma: Abitur.

Professional Experience

01/2003-03/2005 Research associate, Botanic Garden and Botanical Museum Berlin-Dahlem, Freie Universität Berlin, Germany. GBIF-D (Global Biodiversity Information Facility Deutschland), Biodiversity Informatics, Specify-Project

Scholarships
05/2005 - Present
PhD scholarship, German Research Foundation (DFG)
$06 / 2000-11 / 2002$
Student scholarship, German National Academic Foundation (Studientstiftung des deutschen Volkes)
$09 / 1999-04 / 2000$
Exchange student scholarship, University of Osnabrück 


\section{Publications}

\section{Peer reviewed journal \& book publications}

Sobek, S., Kampichler C. \& Weigmann, G. (in press). Diversity and distribution of oribatid mites (Acari, Oribatida) among tree-species and microhabitats in the canopy of a Central European mixed forest. In: Floren, A. and Schmidl, J (eds.). Structure, diversity and functional aspects of arthropod fauna in Central European canopies.

Sobek, S., Scherber, C., Steffan-Dewenter, I. \& Tscharntke, T. (submitted). Sapling herbivory, herbivores and predators across a natural tree diversity gradient in Germany's largest coherent deciduous forest.

Sobek, S., Scherber, C., Steffan-Dewenter, I. \& Tscharntke, T. (submitted). Spatiotemporal changes of canopy beetle communities across a tree diversity gradient.

Sobek, S., Goßner, M., Scherber, C., Steffan-Dewenter, I. \& Tscharntke, T. (submitted). Room for more is in the mix: tree diversity drives spatiotemporal $\beta$-diversity of true bugs (Heteroptera).

Sobek, S., Scherber, C., Schiele, S., Tscharntke, T. \&. Steffan-Dewenter, I. (in prep.). Canopy vs. understory: How does tree diversity affect trap-nesting bee and wasp communities and their natural enemies across forest strata?

Sobek, S., Schuldt, A., Scherber, C., Steffan-Dewenter, I. \& Tscharntke, T. (in prep.). Spiders in the forest canopy: The role of tree diversity for abundance and species richness.

Weigmann, G., Sobek, S. \& C. Kampichler 2004. Eine neue Methode zum Sammeln von rindenbewohnenden Kleinarthropoden aus Baumkronen. Abh. Ber. Naturkundemus. Görlitz. Band 76, Heft 1. S. 93-100.

\section{Conference \& workshop proceedings}

Sobek, S., Steffan-Dewenter, I. \& Tscharntke, T. 2007. Arthropod species richness, herbivory and predator/prey ratios along a tree diversity gradient in a Central European deciduous forest. In: Interactive Abstract Index, ESA/SER Joint Meeting, San Jose, USA.

Sobek, S., Steffan-Dewenter, I. \& Tscharntke, T. 2007. Effects of tree species diversity on insect herbivory and arthropod species richness. In: Verhandlungen der Gesellschaftt für Ökologie (Proceedings of the GfÖ), Band 37, Marburg, Germany p. 57.

Sobek, S., Steffan-Dewenter, I. \& Tscharntke, T. 2006. Tree species richness as a driver for arthropod diversity in the canopy of a temperate deciduous forest. In: Verhandlungen der Gesellschaftt für Ökologie (Proceedings of the GfÖ), Band 36, Bremen, Germany p. 153.

Sobek, S., Kampichler, C. \& Weigmann, G. 2005. Estimating faunal diversity: abundance, species richness and faunal similarity of oribatid mite communities (Acari, Oribatida) in the canopy of a temperate mixed forest. In: 4th International Canopy Conference "Tropical versus Temperate Forests", Leipzig, Germany, p. 10. 
Sobek, S., Kampichler, C. \& Weigmann, G. 2005. Dominance structure and species composition of an oribatid mite community (Acari, Oribatida) in the canopy of a Central European mixed forest. In: 4th International Canopy Conference "Tropical versus Temperate Forests", Leipzig, Germany, p. 145.

Sobek, S. \& Berendsohn, W.G. 2004. Specify software implementation for biological collection management and biodiversity information access. In: GBIF-D: German Participation in the Global Biodiversity Information Facility. Projects in the National German Programme for the Global Biodiversity Information Facility 2003-2006. Status Report 2004, pp. 14-15.

Sobek, S., Zippel, E. \& W. G. Berendsohn 2004. Specify -Testing and adapting a software for biological collection management. In: Botanic Gardens: Awareness for Biodiversity. BGBM, Berlin., pp. 57-58.

Sobek, S. \& Berendsohn, W. G. 2003. SPECIFY - Evaluation and implementation of a software for biological collection management. In: Sustainable use and conservation of biological diversity - a challenge for society, Symposium Report Part A. DLR, Bonn, pp. 234235. 